\title{
Controlled Rough Paths on Manifolds I
}

\author{
Bruce K. Driver* Jeremy S. Semko ${ }^{\dagger}$
}

September 28, 2018

\begin{abstract}
In this paper, we build the foundation for a theory of controlled rough paths on manifolds. A number of natural candidates for the definition of manifold valued controlled rough paths are developed and shown to be equivalent. The theory of controlled rough one-forms along such a controlled path and their resulting integrals are then defined. This general integration theory does require the introduction of an additional geometric structure on the manifold which we refer to as a "parallelism." The transformation properties of the theory under change of parallelisms is explored. Using these transformation properties, it is shown that the integration of a smooth one-form along a manifold valued controlled rough path is in fact well defined independent of any additional geometric structures. We present a theory of push-forwards and show how it is compatible with our integration theory. Lastly, we give a number of characterizations for solving a rough differential equation when the solution is interpreted as a controlled rough path on a manifold and then show such solutions exist and are unique.
\end{abstract}

\section{Contents}

1 Introduction 2

1.1 Summary of Results . . . . . . . . . . . . . . . . . . . . 2

2 Definitions of Controlled Rough Paths with Examples 4

2.1 Review of Euclidean Space Rough Paths . . . . . . . . . . . . . . . . . . 4

2.2 Manifold-Valued Controlled Rough Paths . . . . . . . . . . . . . . . . 9

2.3 Gauges. . . . . . . . . . . . . . . . . . . . . 9

2.3.1 A Covariant Derivative Gives Rise to a Gauge . . . . . . . . . . . . . 14

2.4 Controlled Rough Paths . . . . . . . . . . . . . . . . . . . . 16

2.5 Chart and Gauge CRP Definitions are Equivalent . . . . . . . . . . . . 17

2.5.1 Results Used in Proof of Theorem 2.44 . . . . . . . . . . . . . 17

2.5.2 Proof of Theorem $2.44 \ldots \ldots \ldots \ldots \ldots \ldots \ldots$

2.6 Examples of Controlled Rough Paths . . . . . . . . . . . . . . . . 23

2.7 Smooth Function Definition of CRP . . . . . . . . . . . . . . . . . . . . 25

2010 Mathematics Subject Classification. 58J65; 60H30,

Key words and phrases. Dynamical Systems, Rough Paths.

*The research of Bruce K. Driver was supported in part by NSF Grant DMS-0739164. The author also gratefully acknowledges the generosity and hospitality of the Imperial College Mathematics Department, where part of this research was carried out, and was supported by EPSRC grant EP/M00516X/1 and a Nelder Visiting Fellowship during the Fall of 2014.

${ }^{\dagger}$ The research of Jeremy S. Semko was supported in part by NSF Grant DMS-0739164. 
3 Integration of Controlled One-Forms 25

3.1 Controlled One-Forms Along a Rough Path . . . . . . . . . . . . . . . . . 25

3.2 The Compatibility Tensors . . . . . . . . . . . . . . . . . . 26

$3.3 U$ - Controlled Rough Integration $\ldots \ldots \ldots \ldots \ldots$

3.4 Almost Additivity Result . . . . . . . . . . . . . . . . . . . . . 344

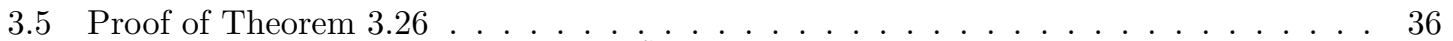

3.6 A Map from $C R P_{y}^{U}(M, V)$ to $C R P_{y}^{\tilde{U}}(M, V) \ldots \ldots \ldots \ldots \ldots \ldots \ldots$

4 Integrating One-Forms Along a CRP 39

4.1 Integration of a One-Form Using Charts . . . . . . . . . . . . . . . . . . . . 41

4.2 Push-forwards of Controlled Rough Paths . . . . . . . . . . . . . . . . 44

5 Rough Differential Equations 47

5.1 RDEs on a Manifold . . . . . . . . . . . . . . . . . . 48

5.1 .1 RDEs from the Gauge Perspective . . . . . . . . . . . . . 52

6 Appendix 53

6.1 Taylor Expansion on a Riemannian manifold . . . . . . . . . . . . . 535

6.2 Equivalence of Riemannian Metrics on Compact Sets . . . . . . . . . . . . . . 56

6.3 Covariant Derivatives on Euclidean Space . . . . . . . . . . . . . . . 556

6.4 Second order gauge inequality does not imply second order chart inequality. . . . . . 58

6.5 Rough Differential Equation Results in Euclidean Space . . . . . . . . . . . . . 559

\section{Introduction}

In a series of papers $20 \sqrt{22}$, Terry Lyons introduced and developed the far reaching theory of rough path analysis. This theory allows one to solve (deterministically) differential equations driven by rough signals at the expense of "enhancing" the rough signal with some additional information. Lyons' theory has found numerous applications to stochastic calculus and stochastic differential equations, for example see [4], [5], [6], [8], and the references therein. For some more recent applications, see [1, [19, [18, [9], and [2.

The rough path theory mentioned above has been almost exclusively developed in the context of state spaces being either finite or infinite dimensional Banach spaces with the two exceptions of 7 and [3]. In [7, a version of manifold valued rough paths is developed in the context of "currents," while in 3 the authors develop a more concrete theory by working with embedded submanifolds.

The purpose of this paper is to define and develop a third interpretation of rough paths on manifolds based on Gubinelli's 14 notions of "controlled" rough paths. As Gubinelli's perspective has proved extremely useful in the flat case (most notably see Hairer [15]), it is expected such a theory of controlled rough paths on manifolds can give new insights as well as applications to the existing literature. We now will present a brief summary of the results contained in this paper.

\subsection{Summary of Results}

Let $M^{d}$ be a $d$-dimensional manifold, $\mathbf{X}_{s, t}:=1+x_{s, t}+\mathbb{X}_{s, t}$ be a weak-geometric rough path in $W:=\mathbb{R}^{k}$ with $1 \leq p<3$. A rough path controlled by $\mathbf{X}$ on $M$ (see Definition 2.35 is a pair of 
continuous functions $y:[0, T] \rightarrow M$, and $y^{\dagger}:[0, T] \rightarrow L(W, T M)$ such that (somewhat imprecisely speaking) for all $0 \leq s \leq t \leq T$;

$$
\text { 1) } \left.y_{s}^{\dagger}: W \rightarrow T_{y_{s}} M, \quad 2\right) \psi\left(y_{s}, y_{t}\right)=y_{s}^{\dagger} x_{s, t}+O\left(\left|x_{s, t}\right|^{2}\right) \text {, and 3) } U\left(y_{s}, y_{t}\right) y_{t}^{\dagger}-y_{s}^{\dagger}=O\left(\left|x_{s, t}\right|\right) \text {, }
$$

where $\psi$ is a "logarithm" on $M$ (see Definition 2.15) and $U$ is a "parallelism" on $M$ (see Definition 2.16. . [When $M=\mathbb{R}^{d}$, one identifies all tangent spaces in which case one typically takes $U(m, n)=$ $I$ and $\psi(m, n)=n-m$.] The pair $\mathcal{G}:=(\psi, U)$ is called a gauge. Alternatively one can define controlled rough paths locally via a chart $\phi$ by requiring (see Definition 2.40)

$$
\phi\left(y_{t}\right)-\phi\left(y_{s}\right)-d \phi \circ y_{s}^{\dagger} x_{s, t}=O\left(\left|x_{s, t}\right|^{2}\right) \text { and } d \phi \circ y_{t}^{\dagger}-d \phi \circ y_{s}^{\dagger}=O\left(\left|x_{s, t}\right|\right) .
$$

It is shown in Theorem 2.44 that these two notions of controlled rough paths agree. Moreover, these manifold-valued rough paths may also be characterized as pairs $\mathbf{y}=\left(y, y^{\dagger}\right)$ whose "push-forwards" under smooth real-valued functions are controlled rough paths on $\mathbb{R}$ (See Theorem 2.57).

Two natural examples of manifold valued controlled rough paths are as follows. 1) If $M^{d}$ is an embedded submanifold (see subsection 2.6) and the path $x_{s} \in W$ happens to lie in $M$ (i.e. $x_{s} \in M$ for all $s$ in $[0, T])$, then $\left(x_{s}, P\left(x_{s}\right)\right)$ is an $M$ - valued rough path controlled by $\mathbf{X}$ where $P(m)$ is orthogonal projection onto $T_{m} M$ (see Example 2.55). 2) If $f: W \rightarrow M^{d} \subseteq \mathbb{R}^{\tilde{k}}$ is smooth, then $\left(f\left(x_{s}\right), f^{\prime}\left(x_{s}\right)\right)$ is a rough path controlled by $\mathbf{X}$ (see Example 2.56).

Now let $\mathcal{G}=(\psi, U)$ be a gauge, $V$ be a Banach space, and $\mathbf{y}=\left(y, y^{\dagger}\right)$ be an $M$ - valued controlled rough path as above. A pair of continuous functions $\alpha:[0, T] \rightarrow L(T M, V)$ and $\alpha^{\dagger}$ : $[0, T] \rightarrow L(W \otimes T M, V)$ is a $U$-controlled (rough) one-form along $y$ with values in a Banach space $V$ provided (see Definition 3.1 for details);

1. $\alpha_{s}: T_{y_{s}} M \rightarrow V$ for all $s$,

2. $\alpha_{s}^{\dagger}: W \otimes T_{y_{s}} M \rightarrow V$ for all $s$,

3. $\alpha_{t} \circ U\left(y_{t}, y_{s}\right)-\alpha_{s}-\alpha_{s}^{\dagger}\left(x_{s, t} \otimes(\cdot)\right)=O\left(\left|x_{s, t}\right|^{2}\right)$, and

4. $\alpha_{t}^{\dagger} \circ\left(I \otimes U\left(y_{t}, y_{s}\right)\right)-\alpha_{s}^{\dagger}=O\left(\left|x_{s, t}\right|\right)$.

To abbreviate notation we write $\boldsymbol{\alpha}_{s}=\left(\alpha_{s}, \alpha_{s}^{\dagger}\right)$. As an example, if $\alpha \in \Omega^{1}(M, V)$ is a smooth one-form on $M$ and $U$ is a parallelism it is shown in Proposition 4.2 how to construct $\alpha_{s}^{\dagger} U$ so that $\boldsymbol{\alpha}_{s}^{U}=\left(\alpha_{s}:=\left.\alpha\right|_{T_{u_{s}} M}, \alpha_{s}^{\dagger U}\right)$ is a $U$-controlled (rough) one-form along $y$.

Theorem 3.21 below constructs the integral, $\int\left\langle\boldsymbol{\alpha}, d \mathbf{y}^{\mathcal{G}}\right\rangle$, of $\boldsymbol{\alpha}$ along $\mathbf{y}=\left(y, y^{\dagger}\right)$. This integral is a standard flat $\boldsymbol{V}$-valued controlled rough path along $\mathbf{X}$ which, as the notation suggests, a priori depends on a choice of gauge, $\mathcal{G}=(\psi, U)$. However, it is shown in Corollary 3.31 that the integral actually only depends on the parallelism, $U$. In Theorem 3.24 (also see Proposition 3.6), we show that the integral, $\int\left\langle\boldsymbol{\alpha}, d \mathbf{y}^{\mathcal{G}}\right\rangle$, satisfies a basic but useful associativity property.

In Theorem 3.32 , it is shown that there are "natural" transformations relating all of the above structures under change of parallelism, $U \rightarrow \tilde{U}$, in such a way that the integral, $\int\left\langle\boldsymbol{\alpha}, d \mathbf{y}^{\mathcal{G}}\right\rangle$, is preserved. Consequently, as shown in Theorem 4.3 if $\alpha \in \Omega^{1}(M, V)$ is a smooth one-form on $M$ with values in $V$ and $\boldsymbol{\alpha}_{s}^{U}=\left(\alpha_{s}:=\left.\alpha\right|_{T_{y_{s}} M}, \alpha_{s}^{\dagger U}\right)$ is the associated $U$-controlled (rough) one-form along $y$, then the resulting integral $\int\left\langle\boldsymbol{\alpha}^{U}, d \mathbf{y}^{\mathcal{G}}\right\rangle$ is in fact independent of both the parallelism, $U$, and the logarithm, $\psi$, used in the construction. Therefore we may simply denote the resulting integral 
by $\int \alpha(d \mathbf{y})$. A gauge independent formula for this integral using charts is given in Corollary 4.7 . It is shown in Theorem 4.15 that if $\alpha \in \Omega^{1}(M, V)$ is a smooth one-form on $M$ and $f: M \rightarrow M$ is a smooth map between two manifolds, then

$$
\int\left(f^{*} \alpha\right)(d \mathbf{y})=\int \alpha\left(d\left(f_{*} \mathbf{y}\right)\right)
$$

where $f_{*} \mathbf{y}:=\left(f \circ y, f_{*} \circ y_{s}^{\dagger}\right)$ is the "push-forward" of $\mathbf{y}$ by $f$ (see Definition 4.11) and $f^{*} \alpha \in$ $\Omega^{1}(\tilde{M}, V)$ is the pull-back of $\alpha$.

In Section 5.1. we discuss the notion of a controlled rough path $\mathbf{y}=\left(y, y^{\dagger}\right)$ solving the rough differential equation (RDE)

$$
d \mathbf{y}_{t}=F_{d \mathbf{X}}\left(y_{t}\right) \quad \text { with } \quad y_{0}=\bar{y}_{0}
$$

when $F: W \rightarrow \Gamma(T M)$. Essentially $\mathbf{y}$ will solve such an equation if the path $y$, when pushed forward by any smooth function $f$, has the correct "Taylor expansion" and $y^{\dagger}$ is the correct derivative, i.e. $y_{s}^{\dagger}=F_{(\cdot)}\left(y_{s}\right)$ (see Definition 5.2. Theorem 5.3 then compiles a list of alternative characterizations for solving an RDE both by approximating solutions and by relating them to familiar flat space rough integrals. Next, the existence and uniqueness of solutions are proved in Theorem 5.4 and Theorem 5.5. Lastly, in Theorem 5.9, we record what it means to solve an RDE when one takes the gauge perspective.

In a sequel to this paper, we will develop notions of parallel translation along a controlled rough path along with rough version of Cartan's rolling and unrolling maps in order to characterize all controlled rough paths on $M$.

\section{Definitions of Controlled Rough Paths with Examples}

\subsection{Review of Euclidean Space Rough Paths}

The presentation here will be brief. For a more thorough development, the reader can refer to many sources, for example [12] or [13].

Throughout this paper, we denote $W=\mathbb{R}^{k}$. Let $1 \leq p<3$ and let

$$
\Delta_{[S, T]}=\{(s, t): S \leq s \leq t \leq T\} .
$$

Definition 2.1 A control $\omega$ is a continuous function $\omega: \Delta_{[0, T]} \rightarrow \mathbb{R}_{+}$which is superadditiv $\oint^{1}$ and such that $\omega(s, s)=0$ for all $s \in[0, T]$.

Definition 2.2 Let $\mathbf{X}=(x, \mathbb{X})$ where

$$
x:[0, T] \rightarrow W \quad \text { and } \quad \mathbb{X}: \Delta_{[0, T]} \rightarrow W \otimes W
$$

and are continuous. Then $\mathbf{X}$ is a p-rough path with control $\omega$ if

1. The Chen identity holds:

$$
\mathbb{X}_{s, u}=\mathbb{X}_{s, t}+\mathbb{X}_{t, u}+x_{s, t} \otimes x_{t, u}
$$

for all $0 \leq s \leq t \leq u \leq T$ where $x_{s, t}:=x_{t}-x_{s}$.

\footnotetext{
${ }^{1}$ To say $\omega$ is superadditive means $\omega(s, t)+\omega(t, u) \leq \omega(s, u)$ for all $0 \leq s \leq t \leq u \leq T$.
} 
2. For all $0 \leq s \leq t \leq T$,

$$
\left|x_{s, t}\right| \leq \omega(s, t)^{1 / p} \quad \text { and } \quad\left|\mathbb{X}_{s, t}\right| \leq \omega(s, t)^{2 / p} .
$$

Further, we say that $\mathbf{X}$ is weak-geometric if the symmetric part of $\mathbb{X}_{s, t}\left(\operatorname{sym}\left(\mathbb{X}_{s, t}\right)\right)$ satisfies the relation

$$
\operatorname{sym}\left(\mathbb{X}_{s, t}\right)=\frac{1}{2} x_{s, t} \otimes x_{s, t} .
$$

Notation 2.3 Let $F_{s, t}$ and $G_{s, t}$ be a pair of functions into a normed space. When it is not important to keep careful track of constants we will often write $F_{s, t} \approx G_{s, t}$ (for any $i \in \mathbb{N}$ ) to indicate that there exists $C<\infty$ and $\delta>0$ such that

$$
\left|F_{s, t}-G_{s, t}\right| \leq C \omega(s, t)^{i / p} \text { for all } 0 \leq s \leq t \leq T \text { with }|t-s| \leq \delta .
$$

In this paper, $V, \tilde{V}$, and $\hat{V}$ will denote Banach spaces, and $L(V, \tilde{V})$ will denote the bounded linear transformations from $V$ to $\tilde{V}$.

Example 2.4 If $x(t) \in C^{\infty}([0, T], V)$ is a smooth curve in $V$ and

$$
\mathbb{X}_{s, t}=\int_{s \leq u \leq v \leq t} d x_{u} \otimes d x_{v}=\int_{s}^{t} x_{s, v} \otimes d x_{v}
$$

then $\mathbf{X}=(x, \mathbb{X})$ is a weak-geometric rough path controlled by $\omega(s, t)=|t-s|$. In this example we could take even take $p=1$.

Definition 2.5 Let $\mathbf{X}$ be a p-rough path on $W \oplus W^{\otimes 2}$ with control $\omega$. The continuous pair $\mathbf{y}:=$ $\left(y, y^{\dagger}\right) \in C([a, b], V) \times C([a, b], L(W, V))$ is a $V$ - valued rough path controlled by $\mathbf{X}$ (denoted $\left.\mathbf{y} \in C R P_{\mathbf{X}}([a, b], V)\right)$ if there exists a $C$ such that

1. $\left|y_{t}-y_{s}-y_{s}^{\dagger} x_{s, t}\right| \leq C \omega(s, t)^{2 / p}$, and

2. $\left|y_{t}^{\dagger}-y_{s}^{\dagger}\right| \leq C \omega(s, t)^{1 / p}$ for all $s \leq t$ in $[0, T]$.

We denote $C R P_{\mathbf{X}}(V):=C R P_{\mathbf{X}}([0, T], V)$ for some fixed $T<\infty$.

The approximations in Definition 2.5 are statements which only need to hold locally because of the following (easy) sewing lemma.

Lemma 2.6 (Sewing Lemma) Let $\mathbf{y}:=\left(y, y^{\dagger}\right) \in C([0, T], V) \times C([0, T], L(W, V))$ and let $0=t_{0}<t_{1}<\ldots<t_{l}=T$ be a partition of $[0, T]$ such that $\left.\mathbf{y}\right|_{\left[t_{i}, t_{i+i}\right]}$ is a rough path controlled by $\left.\mathbf{X}\right|_{\left[t_{i}, t_{i+1}\right]}:=\left(\left.x\right|_{\left[t_{i}, t_{i+1}\right]},\left.\mathbb{X}\right|_{\Delta_{\left[t_{i}, t_{i+1}\right]}}\right)$ for all $0 \leq i \leq l-1$. Then $\mathbf{y}$ is a rough path controlled by $\mathbf{X}$.

Proof. Let $C_{i}$ with $0 \leq i \leq l-1$ be such that

$$
\left|y_{t}-y_{s}-y_{s}^{\dagger} x_{s, t}\right| \leq C_{i} \omega(s, t)^{2 / p} \quad \text { and } \quad\left|y_{t}^{\dagger}-y_{s}^{\dagger}\right| \leq C_{i} \omega(s, t)^{1 / p}
$$


whenever $(s, t) \in \Delta_{\left[t_{i}, t_{i+1}\right]}$. Let $\tilde{C}:=\sum_{i=0}^{l-1} C_{i}$. Then by a telescoping series argument and the fact that $\omega$ is increasing (because it is superadditive), it is clear that

$$
\left|y_{t}^{\dagger}-y_{s}^{\dagger}\right| \leq \tilde{C} \omega(s, t)^{1 / p} \forall(s, t) \in \Delta_{[0, T]} .
$$

Now let $C=(2 l-1) \tilde{C}$. If $(s, t) \in \Delta_{[0, T]}$ then there exists $j$ and $j^{*}$ such that $s \in\left[t_{j}, t_{j+1}\right]$ and $t \in\left[t_{j^{*}}, t_{j^{*}+1}\right]$ with $j \leq j^{*}$. If $j=j^{*}$ then

$$
\left|y_{t}-y_{s}-y_{s}^{\dagger} x_{s, t}\right| \leq C \omega(s, t)^{2 / p}
$$

trivially. Otherwise, we have

$$
\begin{aligned}
y_{t}-y_{s}-y_{s}^{\dagger} x_{s, t}= & \left(y_{t}-y_{t_{j^{*}}}\right)+\left(y_{t_{j+1}}-y_{s}\right)+\sum_{i=j+1}^{j^{*}-1}\left(y_{t_{i+1}}-y_{t_{i}}\right) \\
& -y_{s}^{\dagger} x_{s, t_{j+1}}-y_{s}^{\dagger} x_{t_{j^{*}, t}}-\sum_{i=j+1}^{j^{*}-1} y_{s}^{\dagger} x_{t_{i}, t_{i+1}} \\
= & \left(y_{t}-y_{t_{j^{*}}}-y_{t_{j^{*}}}^{\dagger} x_{t_{j^{*}, t}}\right)+\left(y_{t_{j+1}}-y_{s}-y_{s}^{\dagger} x_{s, t_{j+1}}\right)+\left[y_{t_{j^{*}}}^{\dagger}-y_{s}^{\dagger}\right] x_{t_{j^{*}, t}} \\
& +\sum_{i=j+1}^{j^{*}-1}\left(y_{t_{i+1}}-y_{t_{i}}-y_{t_{i}}^{\dagger} x_{t_{i}, t_{i+1}}\right)+\sum_{i=j+1}^{j^{*}-1}\left[y_{s}^{\dagger}-y_{t_{i}}^{\dagger}\right] x_{t_{i}, t_{i+1}} .
\end{aligned}
$$

Taking absolute values and using the fact that $\omega$ is superadditive, we have that the absolute value of each term on the right (including those within the summations) is bounded by $\tilde{C} \omega(s, t)^{2 / p}$. Thus

$$
\begin{aligned}
\left|y_{t}-y_{s}-y_{s}^{\dagger} x_{s, t}\right| & \leq(2 l-1) \tilde{C} \omega(s, t)^{2 / p} \\
& =C \omega(s, t)^{2 / p}
\end{aligned}
$$

In 14. Theorem 1], the following generalization of Theorem 3.3.1 of [22] is proved.

Theorem 2.7 Let $\mathbf{X}$ be a p-rough path on $W \oplus W^{\otimes 2}$ with control $\omega$ and let $\left(y, y^{\dagger}\right)$ be an $L(W, V)$ - valued rough path controlled by $\mathbf{X}$. Then there exists a $z \in C([0, T], V)$ with $z_{0}=0$ and $a C \geq 0$ such that

$$
\left|z_{t}-z_{s}-y_{s} x_{s, t}-y_{s}^{\dagger} \mathbb{X}_{s, t}\right| \leq C \omega(s, t)^{3 / p}
$$

for all $s \leq t$ in $[0, T]$.

We will more commonly refer to the path $z_{t}$ as $\int_{0}^{t}\left\langle\mathbf{y}_{\tau}, d \mathbf{X}_{\tau}\right\rangle$ and its increment, $z_{s, t}:=z_{t}-z_{s}$, as $\int_{s}^{t}\left\langle\mathbf{y}_{\tau}, d \mathbf{X}_{\tau}\right\rangle$. Theorem 2.9 below is a generalization of Theorem 2.7. but before we state it, we will make a remark about certain identifications of spaces.

Remark 2.8 If $V, \tilde{V}$, and $\hat{V}$ are vector spaces, we can make the identification

$$
L(V, L(\tilde{V}, \hat{V})) \cong L(V \otimes \tilde{V}, \hat{V})
$$


via the map $\Xi: L(V, L(\tilde{V}, \hat{V})) \rightarrow L(V \otimes \tilde{V}, \hat{V})$ given by

$$
\Xi(\alpha)[v \otimes \tilde{v}]=\alpha\langle v\rangle\langle\tilde{v}\rangle .
$$

if $\alpha \in L(V, L(\tilde{V}, \hat{V}))$.

The proof of the following theorem (modulo a reparameterization) may be found in 14 or 12 , Remark 4.11].

Theorem 2.9 Let $\mathbf{X}$ be a p-rough path on $W \oplus W^{\otimes 2}$ with control $\omega$, let $\left(y, y^{\dagger}\right)$ be an $V$ - valued rough path controlled by $\mathbf{X}$ and let $\boldsymbol{\alpha}=\left(\alpha, \alpha^{\dagger}\right)$ be an $L(V, \tilde{V})$ - valued rough path controlled by $\mathbf{X}$ where $\alpha_{s}^{\dagger} \in L(W, L(V, \tilde{V})) \cong L(W \otimes V, \tilde{V})$. Then there exists a $z \in C([0, T], V)$ with $z_{0}=0$ and $a C>0$ such that

$$
\left|z_{t}-z_{s}-\alpha_{s}\left(y_{t}-y_{s}\right)-\alpha_{s}^{\dagger}\left(I \otimes y_{s}^{\dagger}\right) \mathbb{X}_{s, t}\right| \leq C \omega(s, t)^{3 / p}
$$

for all $s \leq t$ in $[0, T]$. Moreover if we let $z_{s}^{\dagger}:=\alpha_{s} \circ y_{s}^{\dagger}$, then $\mathbf{z}_{s}:=\left(z_{s}, z_{s}^{\dagger}\right)$ is a $\tilde{V}$-valued controlled rough path.

The path $z_{t}$ in this case will be denoted $\int_{0}^{t}\left\langle\boldsymbol{\alpha}_{\tau}, d \mathbf{y}_{\tau}\right\rangle$ and we will typically summarize Inequality 2.6. by writing

$$
\int_{s}^{t}\left\langle\boldsymbol{\alpha}_{\tau}, d \mathbf{y}_{\tau}\right\rangle \underset{3}{\approx}\left\langle\boldsymbol{\alpha}_{s}, \mathbf{y}_{s, t}^{\mathbb{X}}\right\rangle:=\alpha_{s} y_{s, t}+\alpha_{s}^{\dagger}\left(I \otimes y_{s}^{\dagger}\right) \mathbb{X}_{s, t}
$$

wherein we let $\mathbf{y}_{s, t}^{\mathbb{X}}$ be the increment process defined by,

$$
\mathbf{y}_{s, t}^{\mathbb{X}}:=\left(y_{s, t},\left(I \otimes y_{s}^{\dagger}\right) \mathbb{X}_{s, t}\right) .
$$

Notice that Theorem 2.7 does indeed follow from Theorem 2.9 upon replacing $\left(\alpha, \alpha^{\dagger}\right)$ by $\left(y, y^{\dagger}\right)$ and $\left(y, y^{\dagger}\right)$ by $\left(x, I_{W}\right)$ in Inequality 2.6 .

Remark 2.10 (Motivations) In order to develop some intuition for the expression appearing on the right side of Eq. 2.7), suppose for the moment that all functions $\mathbf{X},\left(y, y^{\dagger}\right)$, and $\left(\alpha, \alpha^{\dagger}\right)$ are smooth so that $\mathbb{X}$ is given by Eq. (2.4). In this case we want $z_{s, t}$ to be the usual integral $\int_{s}^{t} \alpha_{\tau} \dot{y}_{\tau} d \tau$ and to arrive at the expression in Inequality 2.6. we look for an appropriate second order approximation to the integral. Since $p=1$ now we may conclude

$$
\alpha_{s, \tau}=\alpha_{s}^{\dagger} x_{s, \tau}+O\left((\tau-s)^{2}\right)
$$

and

$$
y_{t}-y_{\tau}=y_{\tau}^{\dagger}\left(x_{t}-x_{\tau}\right)+O\left((t-\tau)^{2}\right) \Longrightarrow \dot{y}_{\tau}=y_{\tau}^{\dagger} \dot{x}_{\tau} .
$$

We have the identity;

$$
\int_{s}^{t} \alpha_{\tau} d y_{\tau}=\int_{s}^{t}\left[\alpha_{s}+\alpha_{s, \tau}\right] \dot{y}_{\tau} d \tau=\alpha_{s} y_{s, t}+\int_{s}^{t} \alpha_{s, \tau} \dot{y}_{\tau} d \tau
$$


The last term on the right hand side is approximated up to an error of size $O\left((t-s)^{3}\right)$ as follows,

$$
\begin{aligned}
\int_{s}^{t} \alpha_{s, \tau} \dot{y}_{\tau} d \tau & =\int_{s}^{t} \alpha_{s, \tau} y_{\tau}^{\dagger} \dot{x}_{\tau} d \tau \\
& =\int_{s}^{t} \alpha_{s}^{\dagger} x_{s, \tau} y_{\tau}^{\dagger} \dot{x}_{\tau} d \tau+O\left((t-s)^{3}\right) \\
& =\int_{s}^{t} \alpha_{s}^{\dagger} x_{s, \tau} y_{s}^{\dagger} \dot{x}_{\tau} d \tau+O\left((t-s)^{3}\right) \\
& =\alpha_{s}^{\dagger}\left(I \otimes y_{s}^{\dagger}\right) \int_{s}^{t} x_{s, \tau} \otimes \dot{x}_{\tau} d \tau+O\left((t-s)^{3}\right) \\
& =\alpha_{s}^{\dagger}\left(I \otimes y_{s}^{\dagger}\right) \mathbb{X}_{s, t}+O\left((t-s)^{3}\right) .
\end{aligned}
$$

Combining Eq. 2.9) and Eq. 2.10) gives the approximate equality,

$$
\int_{s}^{t} \alpha_{\tau} d y_{\tau}=\alpha_{s} y_{s, t}+\alpha_{s}^{\dagger}\left(I \otimes y_{s}^{\dagger}\right) \mathbb{X}_{s, t}+O\left((t-s)^{3}\right) .
$$

Controlled rough paths are also useful in interpreting solutions to rough differential equations. Let $F: V \rightarrow L(W, V)$ be smooth where we will write $F(a) w$ as $F_{w}(a)$. We can then make sense of the rough differential equation

$$
d \mathbf{y}_{t}=F_{d \mathbf{x}_{t}}\left(y_{t}\right)
$$

with initial condition $y_{0}=\bar{y}_{0}$. We will need a bit of notation regarding tensor products before we say what it means to solve such an equation.

Notation 2.11 If $\Xi: W \times W \rightarrow V$ is a bilinear form into a vector space $V$, by the universal property of tensor products, $\Xi$ factors through a unique linear function $\Xi^{\otimes}$ on $W \otimes W$ such that $\Xi^{\otimes}(w \otimes \tilde{w})=\Xi(w, \tilde{w})$ for a simple tensor $w \otimes \tilde{w}$. If $\mathbb{W} \in W \otimes W$ we will abuse notation and write

$$
\left.\Xi(w, \tilde{w})\right|_{w \otimes \tilde{w}=\mathbb{W}}=\left.\Xi(w \otimes \tilde{w})\right|_{w \otimes \tilde{w}=\mathbb{W}}=\Xi^{\otimes}(\mathbb{W}),
$$

where, to be precise, if $\mathbb{W}=\sum w_{i} \otimes \tilde{w}_{i}$ then

$$
\Xi^{\otimes}(\mathbb{W})=\sum \Xi\left(w_{i}, \tilde{w}_{i}\right) .
$$

We say the controlled rough path $\mathbf{y}=\left(y, y^{\dagger}\right)$ defined on ${ }^{2} I_{0}=[0, T)$ or $I_{0}=[0, T]$ solves Eq. 2.11 if for every $[0, b] \subseteq I_{0}$, we have

$$
\begin{aligned}
y_{s, t} \underset{3}{\approx} F_{x_{s, t}}\left(y_{s}\right)+\left.\left(\partial_{F_{w}\left(y_{s}\right)} F_{w}\right)\left(y_{s}\right)\right|_{w \otimes \tilde{w}=\mathbb{X}_{s, t}} \\
y_{s}^{\dagger}=F .\left(y_{s}\right)
\end{aligned}
$$

for all $s, t \in[0, b]$. If in addition $y_{0}=\bar{y}_{0}$, we say $\mathbf{y}$ solves Eq. (2.11) with initial condition $y_{0}=\bar{y}_{0}$.

The existence and uniqueness of solutions (at least of the path $y_{s}$ ) to these differential equations (provided $F$ is sufficiently regular) is due to Lyons 22. Clearly if $y_{s}$ is given, then $y_{s}^{\dagger}$ exists and is uniquely determined by $y_{s}^{\dagger}=F$. $\left(y_{s}\right)$. One may refer to Subsection 6.5 in the Appendix for more results regarding rough differential equations on Euclidean space.

\footnotetext{
${ }^{2}$ Here we allow that $\mathbf{y} \in C R P_{\mathbf{X}}\left(I_{0}, V\right)$ if is in an element of $C R P_{\mathbf{X}}(K, V)$ for every compact interval $K \in I_{0}$.
} 


\subsection{Manifold-Valued Controlled Rough Paths}

Let $M=M^{d}$ be a $d$-dimensional manifold, $T M$ be its tangent space, and $\pi:=\pi_{T M}: T M \rightarrow M$ be the natural projection map. Throughout, let $\mathbf{X}=(x, \mathbb{X})$ be a weak-geometric $p$-rough path on $[0, T]$ with with values in $W \oplus W^{\otimes 2}$ and control $\omega$.

The letters $x$ and $y$ will appear in this paper generally as paths, but occasionally they will refer to arbitrary points in Euclidean space. The context will allow the reader to identify their proper usage.

Notation 2.12 When $M=\mathbb{R}^{d}$ we will identify $T \mathbb{R}^{d}$ with $\mathbb{R}^{d} \times \mathbb{R}^{d}$ via

$$
\mathbb{R}^{d} \times \mathbb{R}^{d} \ni(m, v) \rightarrow v_{m}:=\left.\frac{d}{d t}\right|_{0}(m+t v) \in T_{m} \mathbb{R}^{d}
$$

and, by abuse of notation, we let $\left|v_{m}\right|=|v|$ when $|\cdot|$ is the standard Euclidean norm.

Notation 2.13 Whenever $\phi$ is a map, let $D(\phi)$ and $R(\phi)$ denote the domain and range of $\phi$ respectively. If $\phi \in C^{\infty}\left(M, \mathbb{R}^{d^{\prime}}\right)$ has open domain, let $d \phi: T D(\phi) \longrightarrow \mathbb{R}^{d^{\prime}}$ be defined by

$$
d \phi\left(v_{m}\right):=\left.\frac{d}{d t}\right|_{0} \phi(\sigma(t)) \in \mathbb{R}^{d^{\prime}}
$$

where $\sigma$ is such that $\sigma(0)=m \in D(\phi)$ and $\dot{\sigma}(0)=v_{m} \in T_{m} M$. Denote $d \phi_{m}:=\left.d \phi\right|_{T_{m} M}$. If $f \in C^{\infty}(M, \tilde{M})$ where $\tilde{M}$ is another manifold, we let $f_{*}$ be the push-forward of $f$ so that $f_{*}: T D(f) \longrightarrow T \tilde{M}$ is defined by

$$
f_{*}\left(v_{m}\right):=\left.\frac{d}{d t}\right|_{0} f(\sigma(t)) \in T_{f(m)} \tilde{M}
$$

where again $\dot{\sigma}(0)=v_{m}$. Analogously we let $f_{* m}=\left.f_{*}\right|_{T_{m} M}$. Note that $\phi_{*}\left(v_{m}\right)=\left(\phi(m), d \phi\left(v_{m}\right)\right)=$ $\left[d \phi\left(v_{m}\right)\right]_{\phi(m)} \cdot$

\subsection{Gauges}

Definition 2.14 Let $\mathcal{U}$ be an open set on $M$. An open set $\mathcal{D}^{\mathcal{U}} \subseteq M \times M$ is a $\mathcal{U}$ - diagonal domain if it contains the diagonal of $\mathcal{U}$, that is $\Delta^{\mathcal{U}}:=\bigcup_{m \in \mathcal{U}}(m, m) \subseteq \mathcal{D}^{\mathcal{U}}$. A local diagonal domain is a $\mathcal{V}$ - diagonal domain for some nonempty open $\mathcal{V} \subseteq M$.

If $\mathcal{U}=M$ we write $\mathcal{D}:=\mathcal{D}^{M}$ and refer to $\mathcal{D}$ simply as a diagonal domain.

Throughout the paper, $\mathcal{D}$ will always denote a diagonal domain.

Definition 2.15 A smooth function $\psi: \mathcal{D} \rightarrow T M$ is called a logarithm if:

1. $\psi(m, n) \in T_{m} M$

2. $\psi(m, m)=0_{m}$

3. $\left.\psi(m, \cdot)_{*}\right|_{T_{m} M}=I_{m}$ 
We also write $\psi_{m}$ for $\psi(m, \cdot)$.

If the above holds for $\psi$ defined on a local diagonal domain, we may refer to $\psi$ as a local logarithm.

If $E$ is a any vector bundle, we will denote the smooth sections of $E$ by $\Gamma(E)$. We define $L(T M, T M)$ as the vector bundle $\tilde{E}$ over the manifold $M \times M$ such that $\tilde{E}_{(n, m)}=L\left(T_{m} M, T_{n} M\right)$ and

$$
\tilde{E}=\bigcup\left\{\tilde{E}_{(n, m)}: n, m \in M\right\}
$$

Definition 2.16 A smooth section $U \in \Gamma(L(T M, T M))$ with domain $\mathcal{D}$ (i.e. $U(n, m) \in L\left(T_{m} M, T_{n} M\right)$ for all $(n, m) \in \mathcal{D})$ is called a parallelism if $U(m, m)=I_{m}$. If $U$ is only defined on a local diagonal domain, we refer to $U$ as a local parallelism.

Definition 2.17 We call the pair $\mathcal{G}:=(\psi, U)$ (where $\psi$ and $U$ have common domain $\mathcal{D}$ ) a gauge on the manifold $M$. If $\mathcal{D}$ is replaced by a local diagonal domain, we call $\mathcal{G}$ a local gauge.

Example 2.18 If $M=\mathbb{R}^{d}$, the maps $\psi(x, y)=[y-x]_{x}$ and $U_{x, y} v_{y}=v_{x}$ form the standard gauge on $\mathbb{R}^{d}$.

Example 2.19 One natural example of a gauge comes from any covariant derivative $\nabla$ on TM. The construction is as follows. Choose an arbitrary Riemannian metric $g$ on $M$. If $m, n \in M$ are "close enough", there is a unique vector $v_{m}$ with minimum length such that $n=\exp _{m}^{\nabla}\left(v_{m}\right)$. We denote this vector by $\psi^{\nabla}(m, n):=\left(\exp _{m}^{\nabla}\right)^{-1}(n)$ or by $\exp _{m}^{-1}(n)$ if $\nabla$ is clear from the context. We further let

$$
U^{\nabla}(n, m):=/ / 1\left(t \rightarrow \exp _{m}\left(t \exp _{m}^{-1}(n)\right)\right),
$$

where, for any smooth curve $\sigma:[0,1] \rightarrow M$, we let $/ / s(\sigma)=/ /_{s}^{\nabla}(\sigma): T_{\sigma(0)} M \rightarrow T_{\sigma(s)} M$ denote parallel translation along $\sigma$ up to time $s \in[0,1]$. It is shown in Corollary 2.33 that there is a diagonal domain $\mathcal{D} \subseteq M \times M$ such that $\left(\psi^{\nabla}, U^{\nabla}\right)$ so defined is a gauge on $\mathcal{D}$.

Remark 2.20 We can also get a covariant derivative from a parallelism. If $U$ is a parallelism, then we can define covariant derivative $\nabla^{U}$ on $T M$ by

$$
\nabla_{v_{m}}^{U}(Y):=\left.\frac{d}{d t}\right|_{0} U\left(m, \sigma_{t}\right) Y\left(\sigma_{t}\right)
$$

where $\dot{\sigma}(0)=v_{m}$ and $Y$ is a vector field on $M$.

Remark 2.21 Although the definition of a gauge includes stipulating a $U$, if we have just $\psi$, we can define $U^{\psi}(n, m):=\psi(n, \cdot)_{* m}$ and set $\mathcal{G}^{\psi}:=\left(\psi, U^{\psi}\right)$.

Remark 2.22 We may make a local gauge out of a chart $\phi$. Indeed, we pull back the flat gauge in Example 2.18 to $M$ to define

$$
\begin{aligned}
\psi^{\phi}(m, n) & :=\left(d \phi_{m}\right)^{-1}[\phi(n)-\phi(m)] \\
U^{\phi}(n, m) & :=\left(d \phi_{n}\right)^{-1} d \phi_{m} .
\end{aligned}
$$

This is a gauge which is also consistent with Remark 2.21 and $D\left(\psi^{\phi}\right)=D\left(U^{\phi}\right)=D(\phi) \times D(\phi)$. 
Before moving on to controlled rough paths on manifolds, let us record the structure of the general gauge on $\mathbb{R}^{d}$.

Notation 2.23 If $(\psi, U)$ is a local gauge on $\mathbb{R}^{d}$, then we write $(\bar{\psi}, \bar{U})$ to mean the functions determined by the relations

$$
\psi(x, y)=[\bar{\psi}(x, y)]_{x} \quad \text { and } \quad U(x, y)\left(v_{y}\right)=[\bar{U}(x, y) v]_{x}
$$

so that $\bar{\psi}(x, y) \in \mathbb{R}^{d}$ and $\bar{U}(x, y) \in \operatorname{End}\left(\mathbb{R}^{d}\right)$.

Theorem 2.24 If $\mathcal{G}=(\psi, U)$ is a local gauge on $\mathbb{R}^{d}$, for every open convex subset $\mathcal{V} \subseteq \mathbb{R}^{d}$ such that $\mathcal{V} \times \mathcal{V} \subseteq D(\mathcal{G})$, there exists smoothly varying functions $A(x, y) \in L\left(\left(\mathbb{R}^{d}\right)^{\otimes 2}, \mathbb{R}^{d}\right)$ and $B(x, y) \in$ $L\left(\mathbb{R}^{d}\right.$, End $\left.\left(\mathbb{R}^{d}\right)\right)$ defined for $(x, y) \in \mathcal{V} \times \mathcal{V}$ such that

$$
\begin{aligned}
& \bar{U}(x, y)=I+B(x, y)(y-x), \\
& \bar{\psi}(x, y)=y-x+A(x, y)(y-x)^{\otimes 2}, \\
& B(x, x)=D_{2} \bar{U}(x, x), \text { and } A(x, x)=\frac{1}{2}\left(D_{2}^{2} \bar{\psi}\right)(x, x) .
\end{aligned}
$$

The converse holds as well.

Furthermore, we can find a smoothly varying function $C(x, y) \in L\left(\left(\mathbb{R}^{d}\right)^{\otimes 3}, \mathbb{R}^{d}\right)$ defined on $\mathcal{V} \times \mathcal{V}$ such that

$$
\begin{aligned}
C(x, x) & =\frac{1}{6}\left(D_{2}^{3} \bar{\psi}\right)(x, x), \text { and } \\
\bar{\psi}(x, y) & =y-x+\frac{1}{2}\left(D_{2}^{2} \bar{\psi}\right)(x, x)(y-x)^{\otimes 2}+C(x, y)(y-x)^{\otimes 3} .
\end{aligned}
$$

Proof. Let $x, y$ be points in $\mathcal{V}$. Taylor's theorem with integral remainder applied to the second variable with $x$ fixed gives,

$$
\bar{U}(x, y)=I+\int_{0}^{1}\left(D_{2} \bar{U}\right)(x, x+t(y-x))(y-x) d t
$$

and

$$
\bar{\psi}(x, y)=0+\left(D_{2} \bar{\psi}\right)(x, x)(y-x)+\int_{0}^{1}\left(D_{2}^{2} \bar{\psi}\right)(x, x+t(y-x))(y-x)^{\otimes 2}(1-t) d t
$$

from which Eqs. 2.13 - 2.15 follow with

$$
\begin{aligned}
& B(x, y)=\int_{0}^{1}\left(D_{2} \bar{U}\right)(x, x+t(y-x)) d t \text { and } \\
& A(x, y)=\int_{0}^{1}\left(D_{2}^{2} \bar{\psi}\right)(x, x+t(y-x))(1-t) d t .
\end{aligned}
$$

The converse statement is easy to verify. The proof of Eqs. 2.16) and (2.17) also follow by Taylor's theorem (now to third order) in which case,

$$
C(x, y)=\frac{1}{2} \int_{0}^{1}\left(D_{2}^{3} \bar{\psi}\right)(x, x+t(y-x))(y-x)^{\otimes 2}(1-t)^{2} d t .
$$


Let $B_{r}(x) \subseteq \mathbb{R}^{d}$ be the open ball of radius $r$ centered at $x$.

Remark 2.25 If $\psi$ and $\tilde{\psi}$ are local logarithms on $\mathbb{R}^{d}$, it is easy to check using Theorem 2.24 that for all $\tilde{x} \in \mathbb{R}^{d}$, there exists an $r>0$ and $C>0$ such that $|\psi(x, y)| \leq C|\tilde{\psi}(x, y)|$ for all $x, y \in B_{r}(\tilde{x})$.

We now wish to transfer these local results to the manifold setting. In order to do this we need to develop some notation for stating that two objects on a manifold are "close" up to some order. Let $g$ be any smooth Riemannian metric on $M$.

Notation 2.26 We write $d_{g}$ for the metric associated to $g$ and define $\left|v_{m}\right|_{g}:=\sqrt{g_{m}\left(v_{m}, v_{m}\right)}$ $\forall v_{m} \in T M$. Further, we let $|\cdot|_{g, o p}$ be the operator "norm" induced by $|\cdot|_{g}$ on $L(T M, V)$, i.e. if $f_{m} \in L\left(T_{m} M, V\right)$, then

$$
\left|f_{m}\right|_{g, o p}:=\sup \left\{\left|f_{m}\left\langle v_{m}\right\rangle\right|:\left|v_{m}\right|_{g}=1\right\} .
$$

Definition 2.27 Let $F, G$ be smooth $T M$ [respectively $L(T M, T M)$ ] valued functions with $\mathcal{W}$ diagonal domains. The expression

$$
F(m, n)={ }_{k} G(m, n) \text { on } \mathcal{W}
$$

indicates that for every point in $w \in \mathcal{W}$, there exists an open $\mathcal{O}_{w} \subseteq M$ containing $w$ such that $\mathcal{O}_{w} \times \mathcal{O}_{w} \subseteq D(F) \cap D(G)$ and $a C>0$ such that

$$
|F(m, n)-G(m, n)|_{g,[g, o p]} \leq C\left(d_{g}(m, n)\right)^{k}
$$

for all $m, n \in \mathcal{O}_{w}$.

Occasionally we will omit the reference to $\mathcal{W}$ in which case it we mean the condition 2.19) holds where it makes sense to hold.

Note that in (2.18), the reference to $g$ is not explicit. In fact, the definition does not depend on the choice of $g$ as all Riemannian metrics are locally equivalent. [See Corollary 6.4 in the Appendix for precise statement and proof of this standard fact.]

We may also use the $={ }_{k}$ notation to make statements in regards to other measures of distance:

Corollary 2.28 Let $\mathcal{W}$ be an open subset of $M$ and $g$ and $\tilde{g}$ be any two Riemannian metrics on $M$. If $F(m, n)={ }_{k} G(m, n)$ on $\mathcal{W}$ (so that $F$ and $G$ have $\mathcal{W}$-diagonal domains), then for every local logarithm $\psi$ and $w \in \mathcal{W}$ such that $(w, w) \in D(\psi)$, there exists an open $\mathcal{O}_{w} \subseteq \mathcal{W}$ containing $w$ and $C>0$ such that

$$
|F(m, n)-G(m, n)|_{g,[g, o p]} \leq C|\psi(m, n)|_{\tilde{g}}^{k} \forall m, n \in \mathcal{O}_{w} .
$$

In particular, using the local logarithm $\psi(m, n)=\left(d \phi_{m}\right)^{-1}[\phi(n)-\phi(m)]$, we have that if $w \in$ $D(\phi) \cap \mathcal{W}$, then there exists an $\mathcal{O}_{w} \subseteq D(\phi) \cap \mathcal{W}$ and $a C>0$ such that

$$
|F(m, n)-G(m, n)|_{g,[g, o p]} \leq C|\phi(n)-\phi(m)|^{k} \forall m, n \in \mathcal{O}_{w} .
$$


Proof. The proof of the Corollary will use Remark 2.25 and the local equivalence of any two Riemannian metrics, Corollary 6.4 in the Appendix. First we simplify matters by assuming that we are working in Euclidean space which may be accomplished by pushing the metric and functions forward using charts. Assuming this, we now derive a local inequality that holds for any two logarithms $\psi$ and $\tilde{\psi}$ when $(w, w) \in D(\psi) \cap D(\tilde{\psi})$. Namely, there exist an open neighborhood, $\mathcal{O}_{w}$, of $w$ such that

$$
|\tilde{\psi}(m, n)|_{g} \leq C_{1}|\tilde{\psi}(m, n)| \leq C_{2} C_{1}|\psi(m, n)| \leq C_{3} C_{2} C_{1}|\psi(m, n)|_{\tilde{g}} \forall(m, n) \in \mathcal{O}_{w} \times \mathcal{O}_{w},
$$

where the first and third inequality follow from Corollary 6.4 with one metric being the standard Euclidean metric and the other metric being $g$ or $\tilde{g}$ respectively, and the second inequality is true by Remark 2.25 . Thus, there exists a $\tilde{C}$ such that

$$
|\tilde{\psi}(m, n)|_{g} \leq \tilde{C}|\psi(m, n)|_{\tilde{g}}
$$

Now let $\nabla^{g}$ be the Levi-Civita covariant derivative associated to $g$. By setting $\tilde{\psi}(m, n)=$ $\left(\exp _{m}^{\nabla^{g}}\right)^{-1}(n)$ and shrinking $\mathcal{O}_{w}$ if necessary to ensure that $\left(\exp _{(\cdot)}^{\nabla^{g}}\right)^{-1}(\cdot)$ is defined and injective on $\mathcal{O}_{w} \times \mathcal{O}_{w}$, we have that

$$
\left|\left(\exp _{m}^{\nabla^{g}}\right)^{-1}(n)\right|_{g} \leq \tilde{C}|\psi(m, n)|_{\tilde{g}}
$$

In this setting, $d_{g}(m, n)=\left|\left(\exp _{m}^{\nabla^{g}}\right)^{-1}(n)\right|_{g}$, and since $F(m, n)={ }_{k} G(m, n)$ on $\mathcal{W}$ (by shrinking $\mathcal{O}_{w}$ if necessary), we have

$$
|F(m, n)-G(m, n)|_{g,[g, o p]} \leq \hat{C}\left(d_{g}(m, n)\right)^{k} \quad \forall m, n \in \mathcal{O}_{w}
$$

for some $\hat{C}$. Thus, we have

$$
|F(m, n)-G(m, n)|_{g,[g, o p]} \leq \hat{C}(\tilde{C})^{k}|\psi(m, n)|_{\tilde{g}}^{k} .
$$

which is the statement of the Corollary with $C:=\hat{C}(\tilde{C})^{k}$.

In the sequel, Corollary 2.28 will typically be used without further reference in order reduce the proof of showing $F(m, n)={ }_{k} G(m, n)$ in the manifold setting to a local statement about functions on convex neighborhoods in $\mathbb{R}^{d}$ equipped with the standard Euclidean flat metric structures. The first example of this strategy will already occur in the proof of Corollary 2.29 below. For a general parallelism it is not true that $U(n, m)^{-1}=U(m, n)$, yet $U(m, n)$ is always a very good approximation to $U(n, m)^{-1}$.

Corollary 2.29 If $U$ is a parallelism on a manifold, $M$, then

$$
U(n, m)^{-1}={ }_{2} U(m, n) .
$$

Proof. This is a local statement so we may use Corollary 2.28 to reduce to the case that $M$ is a convex open subset of $\mathbb{R}^{d}$. We then may use Theorem 2.24 to learn

$$
\bar{U}(n, m)^{-1}=(I+[B(n, m)(m-n)])^{-1}=I+[B(n, m)(n-m)]+O\left(|n-m|^{2}\right)
$$


while

$$
\bar{U}(m, n)=(I+[B(m, n)(n-m)]) .
$$

Subtracting these two equations shows,

$$
\begin{aligned}
\bar{U}(n, m)^{-1}-\bar{U}(m, n) & =[B(n, m)-B(m, n)](n-m)+O\left(|n-m|^{2}\right) \\
& =O\left(|n-m|^{2}\right)
\end{aligned}
$$

wherein we have used $B(n, m)-B(m, n)$ vanishes for $m=n$ and therefore is of order $|m-n|$.

\subsubsection{A Covariant Derivative Gives Rise to a Gauge}

Let $\nabla$ be a covariant derivative on $T M$, and $g$ be any fixed Riemannian metric on $M$. Let $G$ : $T M \rightarrow M \times M$ be the function on $T M$ defined by

$$
G\left(v_{m}\right):=\left(m, \exp _{m}^{\nabla}\left(v_{m}\right)\right) \text { for all } v_{m} \in D(G),
$$

where $D(G)$ is the domain of $G$ defined by

$$
D(G):=\left\{v_{m} \in T M: t \rightarrow \exp _{m}^{\nabla}\left(t v_{m}\right) \text { exists for } 0 \leq t \leq 1\right\} .
$$

We will now develop a subset of $D(G)$ for which $G$ is injective. For each $m \in M$, let $\Lambda_{m}$ denote the set of $r>0$ so that $B_{r}\left(0_{m}\right) \subseteq D(G), \exp _{m}^{\nabla}\left(B_{r}\left(0_{m}\right)\right)$ is an open neighborhood of $m$ in $M$, and $\exp _{m}^{\nabla}: B_{r}\left(0_{m}\right) \rightarrow \exp _{m}^{\nabla}\left(B_{r}\left(0_{m}\right)\right)$ is a diffeomorphism (here $B_{r}\left(0_{m}\right)$ is the open ball in $T_{m} M$ centered at $0_{m}$ with radius $r$ ). The fact that $\Lambda_{m}$ is not empty is a consequence of the inverse function theorem and the fact that $\left(\exp _{m}^{\nabla}\right)_{* 0_{m}}=I_{T_{m} M}$ is invertible. We now define $r_{m}:=\sup \Lambda_{m}$ where $r_{m}=\infty$ is possible and allowed. A little thought shows that $\exp _{m}^{\nabla}\left(B_{r_{m}}\left(0_{m}\right)\right)$ is open and $\exp _{m}^{\nabla}: B_{r_{m}}\left(0_{m}\right) \rightarrow \exp _{m}^{\nabla}\left(B_{r_{m}}\left(0_{m}\right)\right)$ is a diffeomorphism, i.e. either $r_{m}=\infty$ or $r_{m} \in \Lambda_{m}$.

Let us now set $\mathcal{C}^{*}:=\cup_{m \in M} B_{r_{m}}\left(0_{m}\right) \subseteq T M$ and let $G^{*}: \mathcal{C}^{*} \rightarrow M \times M$ be the map defined by

$$
G^{*}\left(v_{m}\right):=\left(m, \exp _{m}^{\nabla}\left(v_{m}\right)\right) \text { for all } v_{m} \in \mathcal{C}^{*} .
$$

It is easy to verify that $G^{*}$ is injective.

We will now build our domain $\mathcal{C}$ for which $\left.G\right|_{\mathcal{C}}$ is diffeomorphic onto its range. First we need a simple local invertibility proposition.

Proposition 2.30 Let $G$ be the function defined in $E q$. 2.20). Then for each $m \in M$, there exists open subsets $\mathcal{V}_{m} \subseteq T M$ and $\mathcal{W}_{m} \subseteq M$ such that $0_{m} \in \mathcal{V}_{m}, m \in \mathcal{W}_{m}$, and $\left.G\right|_{\mathcal{V}_{m}}: \mathcal{V}_{m} \rightarrow \mathcal{W}_{m} \times \mathcal{W}_{m}$ is a diffeomorphism.

Proof. As this a local result we may assume that $M=\mathbb{R}^{d}$ and identify $T M$ with $M \times M$. The function $G: T M \rightarrow M \times M$ then takes on the form $G(x, v)=(x, \bar{G}(x, v))$ where $\bar{G}(x, 0)=x$ and $\left(D_{2} \bar{G}\right)(x, 0)=I_{M}$ for all $x \in M$. A simple computation then shows

$$
G^{\prime}(x, 0)=\left[\begin{array}{ll}
I & 0 \\
I & I
\end{array}\right] \text { for all } x \in M
$$

The result now follows by an application of the inverse function theorem. 
Notation 2.31 If $\mathcal{W}$ is an open subset of $M$ and $\epsilon>0$, let $\mathcal{U}(\mathcal{W}, \epsilon)$ be the open subset of $T M$ defined by

$$
\mathcal{U}(\mathcal{W}, \epsilon):=\left\{v \in \pi^{-1}(\mathcal{W}) \subseteq T M:|v|_{g}<\epsilon\right\} .
$$

Theorem 2.32 Let $\mathcal{C}:=\bigcup \mathcal{U}(\mathcal{W}, \epsilon)$ where the union is taken over all open subsets $\mathcal{W} \subseteq M$ and $\epsilon>0$ such that $\mathcal{U}(\mathcal{W}, \epsilon) \subseteq D(G)$ and $\left.G\right|_{\mathcal{U}(\mathcal{W}, \epsilon)}: \mathcal{U}(\mathcal{W}, \epsilon) \rightarrow G(\mathcal{U}(\mathcal{W}, \epsilon))$ is a diffeomorphism. Then $\mathcal{C}$ is an open subset of $T M$ such that $\mathcal{D}:=G(\mathcal{C})$ is open in $M \times M, G: \mathcal{C} \rightarrow \mathcal{D}$ is a diffeomorphism,

$$
\left\{0_{m}: m \in M\right\} \subseteq \mathcal{C} \subseteq \mathcal{C}^{*} \quad \text { and } \quad \Delta^{M}=\{(m, m): m \in M\} \subseteq \mathcal{D} .
$$

Proof. According to Proposition 2.30, for each $m \in M$ there exists an open neighborhood $\mathcal{W}$ of $m \in M$ and $\epsilon>0$ so that $\mathcal{U}(\mathcal{W}, \epsilon) \subseteq D(G)$ and $G: \mathcal{U}(\mathcal{W}, \epsilon) \rightarrow G(\mathcal{U}(\mathcal{W}, \epsilon))$ is a diffeomorphism. From this it follows that $\left\{0_{m}: m \in \mathcal{W}\right\} \subseteq \mathcal{C}$ and $\mathcal{U}(\mathcal{W}, \epsilon) \subseteq \mathcal{C}^{*}$. As $m \in M$ was arbitrary we may conclude $\left\{0_{m}: m \in M\right\} \subseteq \mathcal{C} \subseteq \mathcal{C}^{*}$. It is now easily verified that $G(\mathcal{C})=\cup G(\mathcal{U}(\mathcal{W}, \epsilon))$ is open, $G: \mathcal{C} \rightarrow G(\mathcal{C})$ is a surjective local diffeomorphism and hence is a diffeomorphism as $\left.G\right|_{\mathcal{C}}$ is injective (since $\left.G\right|_{\mathcal{C}^{*}}$ is injective).

Corollary 2.33 Continuing the notation used in Theorem 2.32, we have $\mathcal{D}$ is a diagonal domain and $\psi:=\left.G\right|_{\mathcal{C}} ^{-1}: \mathcal{D} \rightarrow \mathcal{C} \subseteq T M$ is a logarithm. Moreover, if we define

$$
U(m, n):=/ / 1\left(t \longrightarrow \exp ^{\nabla}(t \psi(m, n))\right)^{-1}: T_{n} M \rightarrow T_{m} M
$$

for all $(m, n) \in \mathcal{D}$, then $U$ is a parallelism on $M$.

Proof. The only thing that remains to be proven is that $U(m, n)$ is smoothly varying. This is a consequence of the fact that solutions to ordinary differential equations depend smoothly on their starting points and parameter in the vector fields. To be more explicit in this case, for $a \in \mathbb{R}^{d}$ let $B_{a}^{\nabla}(\mu)=\dot{u}(0)$ where $u(t)=/ /{ }_{t}\left(\exp ^{\nabla}((\cdot) \mu a)\right) \mu$ for $\mu$ in the frame bundle $G L(M)$ over $M$, so that $B_{a}^{\nabla}$ are the $\nabla$ - horizontal vector fields. Now suppose that $w \in M$ is given and $O(m): \mathbb{R}^{d} \rightarrow T_{m} M$ is a local frame defined for $m$ in an open neighborhood $\mathcal{W}$ of $w$. For $v \in \pi^{-1}(\mathcal{W}) \cap \mathcal{C}$ let $\gamma(t)=\exp ^{\nabla}(t v)$ and $u(t):=/ / t(\gamma) O(\pi(v))$. We then have

$$
\begin{aligned}
& \dot{\gamma}(t)=/ / t(\gamma) v=u(t) O(\pi(v))^{-1} v \text { and } \\
& \frac{\nabla u}{d t}=0 \text { with } u(0)=O(\pi(v)) .
\end{aligned}
$$

These equations are equivalent to solving

$$
\dot{u}(t)=B_{O(\pi(v))^{-1} v}^{\nabla}(u(t)) \text { with } u(0)=O(\pi(v))
$$

in which case $\gamma(t)=\pi_{O(M)}(u(t))$ where $\pi_{O(M)}$ is the projection map from $O(M)$ to $M$. We now define $F(v):=u(1)$ provided $v \in \pi^{-1}(\mathcal{W}) \cap \mathcal{C}$. It then follows that $F: \pi^{-1}(\mathcal{W}) \cap \mathcal{C} \rightarrow G L(M)$ is smooth as the solutions to Eq. (2.21) depend smoothly on its starting point and parameter. From this we learn for $(m, n) \in G\left(\pi^{-1}(\mathcal{W}) \cap \mathcal{C}\right)$ that

$$
U(n, m)=F(\psi(m, n)) O(m)^{-1}
$$

is a smooth function of $(m, n)$. 


\subsection{Controlled Rough Paths}

Notation 2.34 Throughout the remainder of this paper, $\mathbf{y}:=\left(y, y^{\dagger}\right)$ denotes a pair of continuous functions, $y \in C([0, T], M)$ and $y^{\dagger} \in C([0, T], L(W, T M))$, such that $y_{s}^{\dagger} \in L\left(W, T_{y_{s}} M\right)$ for all s.

Definition 2.35 Let $(\psi, U)$ be a gauge. The pair $\left(y_{s}, y_{s}^{\dagger}\right)$ is $(\psi, U)$-rough path controlled by $\mathbf{X}$ if there exists $a C>0$ and $\delta>0$ such that

1.

$$
\left|\psi\left(y_{s}, y_{t}\right)-y_{s}^{\dagger} x_{s, t}\right|_{g} \leq C \omega(s, t)^{2 / p}
$$

and

2.

$$
\left|U\left(y_{s}, y_{t}\right) y_{t}^{\dagger}-y_{s}^{\dagger}\right|_{g} \leq C \omega(s, t)^{1 / p}
$$

hold whenever $0 \leq s \leq t \leq T$ and $|t-s| \leq \delta$. Occasionally we will refer to $y_{s}$ as the path and $y_{s}^{\dagger}$ as the derivative process (or Gubinelli derivative).

Remark 2.36 In Definition 2.35 and in the definitions that follow, we use the convention that the $\delta$ is small enough to ensure that all of the expressions are well defined (in particular here it is small enough to ensure $\left.\left(y_{s}, y_{t}\right) \in \mathcal{D}\right)$.

Remark 2.37 Any path $z_{s}$ in Euclidean space naturally gives rise to a two-parameter "increment process," namely $z_{s, t}=z_{t}-z_{s}$. If $\varphi$ is any function such that $\varphi(z, \tilde{z}) \approx \tilde{z}-z$, then it makes sense to define $z_{s, t}^{\varphi}:=\varphi\left(z_{s}, z_{t}\right)$. This serves as motivation for the following notation.

Notation 2.38 Given a gauge, $\mathcal{G}=(\psi, U)$, let $y_{s, t}^{\psi}:=\psi\left(y_{s}, y_{t}\right)$ and $\left(y^{\dagger}\right)_{s, t}^{U}:=U\left(y_{s}, y_{t}\right) y_{t}^{\dagger}-y_{s}^{\dagger}$. These will be referred to as the $\mathcal{G}$-local increment processes of $\left(y, y^{\dagger}\right)$.

Remark 2.39 With Notation 2.38, 2.22) becomes $\left|y_{s, t}^{\psi}-y_{s}^{\dagger} x_{s, t}\right| \leq C \omega(s, t)^{2 / p}$ and 2.23) becomes $\left|\left(y^{\dagger}\right)_{s, t}^{U}\right| \leq C \omega(s, t)^{1 / p}$.

Definition 2.35 gives one possible notion of a controlled rough path on a manifold. We can also define such an object without having to provide a metric or gauge by using charts on the manifold.

Definition 2.40 The pair $\mathbf{y}_{s}=\left(y_{s}, y_{s}^{\dagger}\right)$ is a chart-rough path controlled by $\mathbf{X}$ if for every chart $\phi$ on $M$ and every $[a, b]$ such that $y([a, b]) \subseteq D(\phi)$ we have the existence of $a C_{\phi, a, b} \geq 0$ such that, for all $a \leq s \leq t \leq b$,

1.

$$
\left|\phi\left(y_{t}\right)-\phi\left(y_{s}\right)-d \phi \circ y_{s}^{\dagger} x_{s, t}\right| \leq C_{\phi, a, b} \omega(s, t)^{2 / p}
$$

and

2.

$$
\left|d \phi \circ y_{t}^{\dagger}-d \phi \circ y_{s}^{\dagger}\right| \leq C_{\phi, a, b} \omega(s, t)^{1 / p}
$$

We will denote $C_{\phi, a, b}$ by $C_{\phi}$ when no confusion is likely to arise. 
Notation 2.41 If $\left(y_{s}, y_{s}^{\dagger}\right)$ is a chart rough path and $\phi$ is a chart as in Definition 2.40, we will write $\phi_{*} \mathbf{y}_{s}$ to mean

$$
\phi_{*} \mathbf{y}_{s}:=\phi_{*}\left(y_{s}, y_{s}^{\dagger}\right):=\left(\phi \circ y_{s}, d \phi \circ y_{s}^{\dagger}\right) .
$$

Note that as long as $y$ remains away from the boundary of $D(\phi)$, then $\phi_{*} \mathbf{y}_{s}$ is a controlled rough path on $\mathbb{R}^{d}$. Another way to think of this is that a chart controlled rough path is one which pushes forward to a controlled rough path in $\mathbb{R}^{d}$.

Before moving on, we'll make a few remarks.

Remark 2.42 If $y^{\dagger}$ is any function satisfying the conditions in either of Definitions 2.35 or 2.40, then $s \rightarrow y_{s}^{\dagger}$ is automatically continuous. For example, if $\left(y_{s}, y_{s}^{\dagger}\right)$ satisfies the conditions of a $(\psi, U)$-rough path in Definition 2.35. then the function $t \rightarrow U\left(y_{s}, y_{t}\right) y_{t}^{\dagger}$ is a continuous at $s$ and therefore $t \rightarrow y_{t}^{\dagger}=U\left(y_{s}, y_{t}\right)^{-1} U\left(y_{s}, y_{t}\right) y_{t}^{\dagger}$ is continuous at $s$.

Remark 2.43 If $M=\mathbb{R}^{d}$ and $\phi=I$ then the chart Definition 2.40 reduces to the usual Definition 2.5 of controlled rough paths. In this case, we identify all the tangent spaces with $\mathbb{R}^{d}$ and forget the base point in the derivative process.

\subsection{Chart and Gauge CRP Definitions are Equivalent}

Theorem 2.44 Let $\mathbf{y}:=\left(y, y^{\dagger}\right)$ be a pair of continuous functions as in Notation 2.34. $M$ be a manifold, and $\mathcal{G}=(\psi, U)$ be any gauge on $M$. Then $\mathbf{y}$ is a chart controlled rough path Definition 2.40) if and only if it is a $(\psi, U)$-controlled rough path (Definition 2.35).

Corollary 2.45 We have the equality of sets

$$
\{(\psi, U)-\text { rough paths }\}=\{(\tilde{\psi}, \tilde{U})-\text { rough paths }\}
$$

for any gauges $(\psi, U)$ and $(\tilde{\psi}, \tilde{U})$ on $M$.

Notation 2.46 Let $C R P_{\mathrm{X}}(M)$ be the collection of controlled rough paths in $M$, i.e. pairs of functions $\mathbf{y}=\left(y, y^{\dagger}\right)$ as in Notation 2.34 which satisfy either (and hence both) of Definitions 2.35 or 2.40 .

We will prove Theorem 2.44 after assembling a number of preliminary results that will be needed in the proof and in the rest of the paper.

\subsubsection{Results Used in Proof of Theorem 2.44}

Our first result is a local version of Theorem 2.44 .

Theorem 2.47 Let $\mathcal{G}=(\psi, U)$ be a gauge on $\mathbb{R}^{d}, \mathbf{z}=\left(z, z^{\dagger}\right) \in C\left([a, b], \mathbb{R}^{d}\right) \times C\left([a, b], L\left(W, \mathbb{R}^{d}\right)\right)$, and $\mathcal{W}$ be an open convex set such that $z([a, b]) \subseteq \mathcal{W}$ and $\mathcal{W} \times \mathcal{W} \subseteq D(\mathcal{G})$. Then $\mathbf{z} \in C R P_{\mathbf{X}}\left(\mathbb{R}^{d}\right)$ iff $\mathbf{z}$ is a $(\psi, U)$-rough path controlled by $\mathbf{X}$ with the choice $\delta:=b-a$. 
Proof. Suppose $\mathbf{z} \in C R P_{\mathbf{X}}\left(\mathbb{R}^{d}\right)$. By Theorem 2.24

$$
\bar{\psi}(x, y)=y-x+A(x, y)(y-x)^{\otimes 2} \forall x, y \in \mathcal{W} .
$$

Clearly $A$ is bounded if it is restricted to $x, y$ in the convex hull of $z([a, b])$ (which is compact and contained in $\mathcal{W}$ ). Thus, for all such points, we have there exists a $C_{1}$ such that

$$
|\bar{\psi}(x, y)-(y-x)| \leq C_{1}|y-x|^{2} .
$$

Taking $y=z_{t}$ and $x=z_{s}$ in this inequality shows

$$
\left|\bar{\psi}\left(z_{s}, z_{t}\right)-z_{s, t}\right| \leq C_{1}\left|z_{t}-z_{s}\right|^{2} .
$$

Since $\mathbf{z} \in C R P_{\mathbf{X}}\left(\mathbb{R}^{d}\right)$, there exists a $C_{2}$ such that

$$
\begin{aligned}
\left|z_{s, t}-z_{s}^{\dagger} x_{s, t}\right| & \leq C_{2} \omega(s, t)^{2 / p} \\
\left|z_{s, t}^{\dagger}\right| & \leq C_{2} \omega(s, t)^{1 / p} .
\end{aligned}
$$

By enlarging $C_{2}$ if necessary we may further conclude,

$$
\left|z_{s, t}\right| \leq C_{2} \omega(s, t)^{1 / p} .
$$

Using Eqs. 2.28 and 2.30 in Eq. 2.27) gives the existence of a $C_{3}<\infty$ such that

$$
\left|\bar{\psi}\left(z_{s}, z_{t}\right)-z_{s}^{\dagger} x_{s, t}\right| \leq C_{3} \omega(s, t)^{2 / p} .
$$

By Theorem 2.24 once more, we have

$$
\bar{U}(x, y)=I+B(x, y)(y-x) .
$$

As was the case for $A, B$ is bounded on the convex hull of $z([a, b])$ so that there exists a $C_{4}$ such that

$$
\begin{aligned}
\left|\bar{U}\left(z_{s}, z_{t}\right) z_{t}^{\dagger}-z_{s}^{\dagger}\right| & \leq\left|z_{s, t}^{\dagger}\right|+C_{4}\left|z_{s, t}\right| \\
& \leq\left(C_{2}+C_{4} C_{2}\right) \omega(s, t)^{1 / p}
\end{aligned}
$$

Thus $\mathbf{z}$ is a $(\psi, U)$-rough path controlled by $\mathbf{X}$ with the choice $\delta:=b-a$ where our $C:=$ $\max \left\{C_{1}, C_{2}\left(1+C_{4}\right)\right\}$.

For the converse direction, suppose $\mathbf{z}$ is a $(\psi, U)$-rough path controlled by $\mathbf{X}$ with the choice $\delta:=b-a$ as in Definition 2.35 From Eq. 2.26) and the triangle inequality we have

$$
|y-x| \leq C_{1}|y-x|^{2}+|\bar{\psi}(x, y)| .
$$

Taking $x=z_{s}$ and $y=z_{t}$ in this inequality and using Definition 2.35 we may find $C_{2}<\infty$ such that

$$
\begin{aligned}
\left|z_{s, t}\right| & \leq C_{1}\left|z_{s, t}\right|^{2}+\left|\psi\left(z_{s}, z_{t}\right)\right| \\
& \leq C_{1}\left|z_{s, t}\right|^{2}+C_{2} \omega(s, t)^{1 / p}
\end{aligned}
$$


for all $s \leq t$ in $[a, b]$. By the uniform continuity of $z$ on $[a, b]$, there exists $\epsilon>0$ such that $C_{1}\left|z_{s, t}\right| \leq \frac{1}{2}$ when $|t-s| \leq \epsilon$ which combined with the previous inequality implies

$$
\left|z_{s, t}\right| \leq 2 C_{2} \omega(s, t)^{1 / p} \text { when }|t-s| \leq \epsilon .
$$

For general $a \leq s \leq t \leq b$ we may write $z_{s, t}$ as a sum of at most $n \leq(b-a) / \epsilon$ increments whose norms are bounded by $2 C_{2} \omega(s, t)^{1 / p}$ wherein we have repeatedly used the estimate above along with the monotonicity of $\omega$ resulting from superactivity. Thus we conclude, with $C_{3}:=2 C_{2}(b-a) / \epsilon<$ $\infty$, that

$$
\left|z_{s, t}\right| \leq C_{3} \omega(s, t)^{1 / p} \forall s, t \in[a, b] .
$$

This estimate along with the inequality in Eq. (2.26) gives,

$$
\left|\bar{\psi}\left(z_{s,} z_{t}\right)-z_{s, t}\right| \leq C_{1}\left|z_{s, t}\right|^{2} \leq C_{1} C_{3}^{2} \omega(s, t)^{2 / p} \forall s, t \in[a, b] .
$$

The previous inequality along with the assumption that $\mathbf{z}$ is a $(\psi, U)$-rough path shows there exists $C_{4}<\infty$ such that

$$
\left|z_{s, t}-z_{s}^{\dagger} x_{s, t}\right| \leq\left|z_{s, t}-\bar{\psi}\left(z_{s,} z_{t}\right)\right|+\left|\bar{\psi}\left(z_{s}, z_{t}\right)-z_{s}^{\dagger} x_{s, t}\right| \leq C_{4} \omega(s, t)^{2 / p} .
$$

From Eq. 2.31, there exists a $C_{5}$ such that

$$
\left|z_{s, t}^{\dagger}\right| \leq\left|U\left(z_{s}, z_{t}\right) z_{t}^{\dagger}-z_{s}^{\dagger}\right|+C_{5}\left|z_{s, t}\right|
$$

This inequality along with the assumption that $\mathbf{z}$ is a $(\psi, U)$-rough path shows there exists $C_{6}<\infty$ such that $\left|z_{s, t}^{\dagger}\right| \leq C_{6} \omega(s, t)^{1 / p}$ for all $a \leq s \leq t \leq b$. Thus we have shown $\mathbf{z} \in C R P_{\mathbf{X}}\left(\mathbb{R}^{d}\right)$.

The rest of this section is now devoted to a number of "stitching" arguments which will be used to piece together a number of local versions of Theorem 2.44 over subintervals as described in Theorem 2.47 into the full global version as stated in Theorem 2.44. For the rest of this section let $\mathcal{X}$ be a topological space and $0 \leq S<T<\infty$.

Lemma 2.48 If $y:[S, T] \rightarrow \mathcal{X}$ is continuous and $y([S, T]) \subseteq \bigcup_{\alpha \in A} \mathcal{O}_{\alpha}$ where $\left\{\mathcal{O}_{\alpha}\right\}_{\alpha \in A}$ is a collection of open subsets of $\mathcal{X}$, then there exists a partition of $[S, T], S=t_{0}<t_{1}<\ldots<t_{l}=T$, and $\alpha_{i} \in A$ such that for all $i$ less than $l$, we have

$$
y\left(\left[t_{i}, t_{i+1}\right]\right) \subseteq \mathcal{O}_{\alpha_{i}}
$$

Proof. Define $T^{*}:=\sup \{t: S \leq t \leq T$, the conclusion of the theorem holds for $[S, t]\}$. Note that trivially $T^{*}>S$. For sake of contradiction, suppose $T^{*}<T$. Then there exists an $\epsilon>0$ such that $T^{*}+\epsilon<T, T^{*}-\epsilon>S$ and $y\left(T^{*}-\epsilon, T^{*}+\epsilon\right) \subset \mathcal{O}_{\alpha^{*}}$ for some $\alpha^{*}$. But the condition of the theorem holds for $T^{*}-\epsilon$ for some partition $P$. By appending $P$ with $T^{*}+\lambda \epsilon$ with $\lambda \in(-1,1]$ we have that $T^{*} \geq T^{*}+\epsilon$ which is absurd. Thus, we must have that $T^{*}=T$.

Definition 2.49 The set $\left\{a_{i}, b_{i}\right\}_{i=0}^{l} \subset[S, T]$ is an interlaced cover of $[S, T]$ if $S=a_{0}<a_{1}<$ $b_{0}<a_{2}<b_{1}<a_{3}<b_{2}<\ldots<a_{l}<b_{l-1}<b_{l}=T$. Let $y:[S, T] \rightarrow \mathcal{X}$. The set $\left\{a_{i}, b_{i}\right\}_{i=0}^{l}$ is an interlaced cover for $y$ if $\left\{a_{i}, b_{i}\right\}_{i=0}^{l}$ is an interlaced cover of $[S, T]$ and $y\left(a_{i+1}\right) \neq y\left(b_{i}\right)$ for all $i$ less than $l$. 


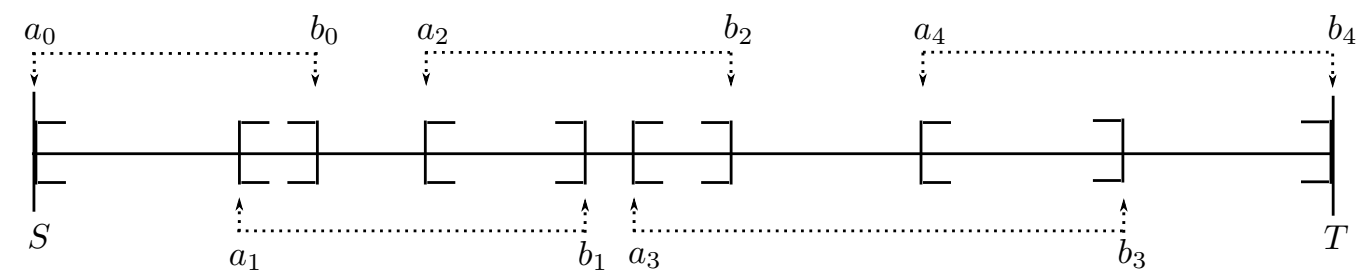

Figure 1: An interlaced cover of $[S, T]$

Corollary 2.50 Suppose $y:[S, T] \rightarrow \mathcal{X}$ is continuous and $y([S, T]) \subseteq \bigcup_{\alpha \in A} \mathcal{O}_{\alpha}$ where $\left\{\mathcal{O}_{\alpha}\right\}_{\alpha \in A}$ is a collection of open sets $\mathcal{O}_{\alpha}$. There exists an interlaced cover for $y,\left\{a_{i}, b_{i}\right\}_{i=0}^{l}$ such that $y\left(\left[a_{i}, b_{i}\right]\right) \subseteq$ $\mathcal{O}_{\alpha_{i}}$. Note that for such a setup, this implies $y\left(\left[a_{i+1}, b_{i}\right]\right) \subseteq \mathcal{O}_{\alpha_{i}} \cap \mathcal{O}_{\alpha_{i+1}}$

Proof. The first step will be a technical one to get rid of unnecessary endpoints. Let $t_{i}^{\prime}$ and $\alpha_{i}^{\prime}$ be as given in Lemma 2.48. Then clearly $y\left(t_{i}^{\prime}\right) \in \mathcal{O}_{\alpha_{i-1}^{\prime}} \cap \mathcal{O}_{\alpha_{i}^{\prime}}$ for all $1 \leq i<l^{\prime}$. Starting with $t_{1}^{\prime}$, we check if $y\left(\left[t_{0}^{\prime}, t_{1}^{\prime}\right]\right) \subseteq \mathcal{O}_{\alpha_{1}}$. In the case it is, we may renumber our partition after removing $t_{1}^{\prime}$ and $\mathcal{O}_{\alpha_{0}^{\prime}}$ to get a new set of $t_{j}^{\prime}$ and $\alpha_{j}^{\prime}$ which still satisfy the result of the lemma. Continuing this process inductively, we may assume that we have such a set $\left\{t_{i}, \alpha_{i}\right\}_{i=0}^{l}$ such that $y\left(\left[t_{i}, t_{i+1}\right]\right)$ is not contained in $\mathcal{O}_{\alpha_{i+1}}$.

To construct the desired interlaced cover, we define $b_{i}:=t_{i+1}$ for all $i \leq l:=l^{\prime}-1$ and $a_{0}:=S$. Note for now that this means $y\left(\left[b_{i-1}, b_{i}\right]\right) \subseteq \mathcal{O}_{\alpha_{i}}$. Then we define the "lower end" stopping time $T_{i}$ for all $i>0$ by the formula

$$
T_{i}:=\inf \left\{t<b_{i}: y\left(\left[t, b_{i}\right]\right) \subseteq \mathcal{O}_{\alpha_{i+1}}\right\} .
$$

By construction and because we refined our partition, $b_{i-1} \leq T_{i}<b_{i}$. It is clear that $y\left(T_{i}\right) \neq y\left(b_{i}\right)$ by the continuity of $y$. Thus, there exists a time $T_{i}^{*}$ such that $T_{i}<T_{i}^{*}$ and $y\left(T_{i}^{*}\right) \neq y\left(b_{i}\right)$. Define

$$
a_{i+i}:=T_{i}^{*}
$$

for all $0<i<n$. Since $y\left(\left[b_{i-1}, b_{i}\right]\right) \subseteq \mathcal{O}_{\alpha_{i}}$ and $a_{i}>b_{i-1}$, we have that $y\left(\left[a_{i}, b_{i}\right]\right) \subseteq \mathcal{O}_{\alpha_{i}}$.

Since the following patching trick will be used multiple times in later proofs, we will prove it here in more generality to avoid too much indexing notation later.

Lemma 2.51 Let $\omega$ be a control and $\left\{a_{i}, b_{i}\right\}_{i=0}^{l}$ be an interlaced cover of $[S, T]$ such that $\omega\left(a_{i+1}, b_{i}\right)>$ 0 for all $i<n$. Let $\theta>0$ and $F: D \rightarrow[0, \infty)$ be a bounded function such that $D \subseteq \Delta_{[S, T]}$ and for each $1 \leq i \leq l$ there exists $C_{i}<\infty$ such that

$$
F(s, t) \leq C_{i} \omega(s, t)^{\theta} \text { for all }(s, t) \in \Delta_{\left[a_{i}, b_{i}\right]} \cap D .
$$

Then there exists a $\tilde{C}<\infty$ such that

$$
F(s, t) \leq \tilde{C} \omega(s, t)^{\theta} \forall(s, t) \in D .
$$

Proof. Let

$$
\begin{aligned}
m & :=\min \left\{\omega\left(a_{i+1}, b_{i}\right)^{\theta}: 0 \leq i<n\right\}, \\
C & :=\max \left\{C_{i}: 0 \leq i \leq n\right\}, \text { and } \\
M & :=\sup \{F(s, t):(s, t) \in D\}<\infty
\end{aligned}
$$


and then define $\tilde{C}:=\max \left\{\frac{M}{m}, C\right\}$. We claim that Inequality 2.32 holds.

If there exists an $i$ such that $s, t \in\left[a_{i}, b_{i}\right] \cap D$, then 2.32 holds trivially. Otherwise, let $i^{*}$ be the largest $i$ such that $s \in\left[a_{i}, b_{i}\right]$. Then $s<a_{i^{*}+1}$ and $t>b_{i^{*}}$. However this says that $[s, t] \supset\left[a_{i^{*}+1}, b_{i^{*}}\right]$ so that

$$
F(s, t) \leq M=\frac{M}{m} m \leq \tilde{C} \omega\left(a_{i^{*}+1}, b_{i^{*}}\right)^{\theta} \leq \tilde{C} \omega(s, t)^{\theta} .
$$

\subsubsection{Proof of Theorem 2.44}

The recurring strategy here will be localize appropriately to work in the $\mathbb{R}^{d}$ case so that we may apply Theorem 2.47. We must choose these localizations carefully so that we may patch the estimates together (with two different strategies) using the lemmas above. One method of patching is a bit more involved than the other; therefore we will present it more formally:

Remark 2.52 (Proof Strategy) Let $y:[a, b] \rightarrow M$ be the first component of $\left(y, y^{\dagger}\right)$ where $\left(y, y^{\dagger}\right)$ is either a $(\psi, U)$ - controlled rough path or chart controlled rough path. Also suppose for each $m \in y([a, b])$, we are given an open neighborhood, $\mathcal{W}_{m} \subseteq M$, of $m$. By Corollary 2.50, there exists an interlaced cover for $y,\left\{a_{i}, b_{i}\right\}_{i=1}^{l}$ and $\left\{m_{i}\right\}_{i=1}^{l}$ such that $y\left(\left[a_{i}, b_{i}\right]\right) \subseteq \mathcal{W}_{m_{i}}$ and $\omega\left(a_{i+1}, b_{i}\right)>0$. Thus, if $F: D \rightarrow[0, \infty)$ is a bounded function such that $D \subseteq \Delta_{[a, b]}$, then in order to prove that

$$
F(s, t) \leq C \omega(s, t)^{\theta} \forall(s, t) \in D,
$$

it suffices to prove; for each $1 \leq i \leq l$ there exists $C_{i}<\infty$ such that

$$
F(s, t) \leq C_{i} \omega(s, t)^{\theta} \text { for all }(s, t) \in \Delta_{\left[a_{i}, b_{i}\right]} \cap D .
$$

Therefore in attempting to prove an assertion in the form of Inequality (2.33), we may assume, without loss of generality, that $y([a, b]) \subseteq \mathcal{W}$ where the $\mathcal{W}$ will have nice properties dependent on our setting.

The proof of Theorem 2.44 will consist of two steps:

1. If gauge conditions of 2.22 and 2.23 hold for some $C>0$ and $\delta>0$, then the chart conditions of 2.24) and 2.25) hold. We will reduce this to the $\mathbb{R}^{d}$ case immediately, then use Lemma 2.6 to patch the estimates together.

2. If the chart condition of (2.24) and (2.25) hold, then gauge condition of $(2.22)$ and $(2.23)$ hold for an appropriately chosen $\delta$. Here we will first show which local estimates we need to satisfy to use Remark 2.52 and then reduce to the $\mathbb{R}^{d}$ case.

In simple terms, step 1 is "localize then patch" and step 2 is "cut nicely, localize, then patch". Proof of Theorem 2.44. Step 1: Definition 2.35 $\Longrightarrow$ Definition 2.40.

We'll first assume that the gauge definition holds, i.e. that there exists a $\delta>0$ and a $C_{1}>0$ such that

$$
\left|\psi\left(y_{s}, y_{t}\right)-y_{s}^{\dagger} x_{s, t}\right|_{g} \leq C_{1} \omega(s, t)^{2 / p}
$$

and

$$
\left|U\left(y_{s}, y_{t}\right) y_{t}^{\dagger}-y_{s}^{\dagger}\right|_{g} \leq C_{1} \omega(s, t)^{1 / p}
$$


hold for all $0 \leq s \leq t \leq T$ such that $|t-s| \leq \delta$. Let $\phi$ be a chart on $M$ and let $[a, b]$ be such that $y([a, b]) \subseteq D(\phi)$. If we define

$$
\begin{aligned}
\psi^{\phi}(x, y) & :=\phi_{*} \psi\left(\phi^{-1}(x), \phi^{-1}(y)\right) \\
U^{\phi}(x, y) & :=\phi_{*} U\left(\phi^{-1}(x), \phi^{-1}(y)\right) \circ\left(\phi_{*}^{-1}\right)_{\phi(y)} \\
\left(z_{s}, z_{s}^{\dagger}\right) & :=\phi_{*}\left(\mathbf{y}_{s}\right)=\left(\phi\left(y_{s}\right), d \phi \circ y_{s}^{\dagger}\right)
\end{aligned}
$$

then it is clear that there exists a $C_{2}=C_{2}\left(\phi_{*}\right)$ such that

$$
\begin{aligned}
\left|\bar{\psi}^{\phi}\left(z_{s}, z_{t}\right)-z_{s}^{\dagger} x_{s, t}\right| & \leq C_{2} \omega(s, t)^{2 / p} \\
\left|\bar{U}^{\phi}\left(z_{s}, z_{t}\right) z_{t}^{\dagger}-z_{s}^{\dagger}\right| & \leq C_{2} \omega(s, t)^{1 / p}
\end{aligned}
$$

for all $a \leq s \leq t \leq b$ such that $t-s \leq \delta$ where $\left(\psi^{\phi}, U^{\phi}\right)$ is a local gauge on $\mathbb{R}^{d}$ and $\left(\bar{\psi}^{\phi}, \bar{U}^{\phi}\right)$ is consistent with Notation 2.23 . Thus $\left(z, z^{\dagger}\right)$ is a $\left(\psi^{\phi}, U^{\phi}\right)$-rough path controlled by $\mathbf{X}$. Finally we need to use this information to show there exists a $C_{\phi, a, b}$ such that

$$
\left|z_{t}-z_{s}-z_{s}^{\dagger} x_{s, t}\right| \leq C_{\phi, a, b} \omega(s, t)^{2 / p} .
$$

and

$$
\left|z_{t}^{\dagger}-z_{s}^{\dagger}\right| \leq C_{\phi, a, b} \omega(s, t)^{1 / p}
$$

for all $s, t$ such that $a \leq s \leq t \leq b$.

In light of the Sewing Lemma 2.6 and Lemma 2.48 , we only need to show that for each $u \in[a, b]$, the inequalities 2.37) and 2.38 hold with $C_{\phi, a, b}$ replaced with $C_{u}$ for all $s, t \in\left(u-\delta_{u}, u+\delta_{u}\right) \cap$ $[a, b]$ such that $s \leq t$ for some $\delta_{u}>0$.

For any $u \in[a, b]$, let $\mathcal{W}_{u}$ be an open convex set of $z_{u}$ such that $\mathcal{W}_{u} \times \mathcal{W}_{u} \subseteq D\left(\psi^{\phi}\right)$. We then choose $\delta_{u}>0$ to be such that $z\left(\left[u-\delta_{u}, u+\delta_{u}\right] \cap[a, b]\right) \subseteq \mathcal{W}_{u}$ and $2 \delta_{u} \leq \delta$. However, now we are in the setting of Theorem 2.47 and are therefore finished with this step.

Step 2: Definition 2.40 $\Longrightarrow$ Definition 2.35

Suppose that the chart item (2.24) holds. We must prove that there exists a $\delta, C>0$ such that

$$
\begin{aligned}
\left|\psi\left(y_{s}, y_{t}\right)-y_{s}^{\dagger} x_{s, t}\right|_{g} & \leq C \omega(s, t)^{2 / p} \\
\left|U\left(y_{s}, y_{t}\right) y_{t}^{\dagger}-y_{s}^{\dagger}\right|_{g} & \leq C \omega(s, t)^{1 / p}
\end{aligned}
$$

for all $s \leq t$ such that $|t-s| \leq \delta$.

We choose $\delta$ such that $|t-s| \leq \delta$ for $0 \leq s \leq t \leq T$ implies that both $\left|\psi\left(y_{s}, y_{t}\right)\right|_{g}$ and $\left|U\left(y_{s}, y_{t}\right)\right|_{g}$ make sense and are bounded. Around every point $m$ of $y([0, T])$, there exists an open $\mathcal{O}_{m}$ containing $m$ and such that $\mathcal{O}_{m} \times \mathcal{O}_{m} \subseteq \mathcal{D}$. Additionally there exists a chart $\phi^{m}$ such that $m \in D\left(\phi^{m}\right)$. By considering an open ball around $\phi^{m}(m)$ in $R\left(\phi^{m}\right)$ and shrinking the radius, we may assume that $\mathcal{V}_{m}:=D\left(\phi^{m}\right) \subseteq \mathcal{O}_{m}$ and the range, $\mathcal{W}_{m}:=\phi\left(\mathcal{V}_{m}\right)$, of $\phi^{m}$ is convex. Since $\left\{\mathcal{V}_{m}\right\}_{m \in y([0, T])}$ is an open cover of $y([0, T])$, we may use this cover along with $D=$ $\{(s, t): 0 \leq s \leq t \leq T$ and $|t-s| \leq \delta\}$ to employ the proof strategy in Remark 2.52. We will do this twice, with $F(s, t)=\left|\psi\left(y_{s}, y_{t}\right)-y_{s}^{\dagger} x_{s, t}\right|_{g}$ in the first iteration and $F(s, t)=\left|U\left(y_{s}, y_{t}\right) y_{t}^{\dagger}-y_{s}^{\dagger}\right|_{g}$ in the second; this will reduce us to considering the case where there exists a single chart $\phi$ such that $y([0, T]) \subseteq D(\phi), D(\phi) \times D(\phi) \subseteq \mathcal{D}$ and $\mathcal{W}=R(\phi)$ is convex. 
Now that we have reduced to a single chart $\phi$, we may define $\left(\psi^{\phi}, U^{\phi}\right)$ and the path $\left(z, z^{\dagger}\right)$ as in Step 1. Then $z([0, T]) \subseteq \mathcal{W}$ and $\mathcal{W} \times \mathcal{W} \subseteq D\left(\psi^{\phi}\right)=D\left(U^{\phi}\right)$. However, by Theorem 2.47 we have that the proper estimates hold because $\mathbf{z}$ is a $\left(\psi^{\phi}, U^{\phi}\right)$-rough path controlled by $\mathbf{X}$. Therefore, we are finished by patching using Remark 2.52 .

Remark 2.53 In the proof of Theorem 2.44, we would have been able to show (and did so somewhat indirectly) that Inequality (2.24) implies Inequality (2.22) for some $\delta>0$. However, it is not true in general that, for a fixed $\delta$, Inequality 2.22) implies Inequality 2.24). See Example 6.7 in the Appendix for a counterexample.

In situations in which we are given a covariant derivative $\nabla$ on a manifold, by Example 2.19 , we have an equivalent definition:

Example 2.54 The pair $\left(y_{s}, y_{s}^{\dagger}\right)$ is an element of $C R P_{\mathbf{X}}(M)$ if and only if there exists a $C$ such that

1.

$$
\left|\left(\exp _{y_{s}}^{\nabla}\right)^{-1}\left(y_{t}\right)-y_{s}^{\dagger} x_{s, t}\right|_{g} \leq C \omega(s, t)^{2 / p}
$$

2.

$$
\left|U_{y_{s}, y_{t}}^{\nabla} y_{t}^{\dagger}-y_{s}^{\dagger}\right|_{g} \leq C \omega(s, t)^{1 / p}
$$

where $\left(\exp _{m}^{\nabla}\right)^{-1}$ and $U_{n, m}^{\nabla}$ are defined as in Example 2.19 and the inequalities hold when $\left(y_{s}, y_{t}\right)$ are in the domain $\mathcal{D}$ as given in Theorem 2.32. In particular, on a Riemannian manifold we can use this definition with the Levi-Civita covariant derivative.

Before providing yet another equivalent definition of controlled rough paths on manifolds, we will present some examples.

\subsection{Examples of Controlled Rough Paths}

Recall $\mathbf{X}=(x, \mathbb{X})$ is a weak-geometric rough path with values in $W \oplus W^{\otimes 2}$ where $W=\mathbb{R}^{k}$. The results here will rely on basic approximations found in the Appendix, Section 6 .

Example 2.55 Let $M^{d} \subseteq W$ be an embedded submanifold and for every $m \in M^{d}$, let $P(m)$ be the orthogonal projection onto the tangent space $T_{m} M$. Suppose $x_{s} \in M^{d}$ for all $s$ in $[0, T]$. Then $\left(x_{s}, P\left(x_{s}\right)\right) \in C R P_{\mathbf{X}}(M)$.

Proof. We will use the gauge as given in Example 2.54 where the $\nabla$ is the Levi-Civita covariant derivative from the induced metric from Euclidean space. Verifying that $P\left(x_{s}\right)$ lives in the correct space is trivial.

Next, to show Inequality 2.39 is satisfied, we use item 1 of Lemma 6.2 which says

$$
\exp _{m}^{-1}(\tilde{m})=P(m)(\tilde{m}-m)+O\left(|\tilde{m}-m|^{3}\right) \text { for all } m \in M^{d} .
$$

Letting $m=x_{s}$ and $\tilde{m}=x_{t}$, we are done. 
Inequality 2.40 is also satisfied as a result of Lemma 6.2 which says that $U_{\tilde{m}, m}^{\nabla}=P(m)+$ $O(|\tilde{m}-m|)$. Thus

$$
\begin{aligned}
P\left(x_{t}\right)-U_{x_{t}, x_{s}}^{\nabla} P\left(x_{s}\right) & \underset{1}{\approx} P\left(x_{t}\right)-P\left(x_{s}\right) P\left(x_{s}\right) \\
& =P\left(x_{t}\right)-P\left(x_{s}\right) \\
& \approx 0
\end{aligned}
$$

The next example will be proved in more generality in Section 4.2. However, we find it instructive to prove it without charts and in the embedded context where the reader may be more comfortable.

Example 2.56 Let $f$ be a smooth function from $W$ to an embedded manifold $\tilde{M}^{d} \subseteq \mathbb{R}^{\tilde{k}}$. Then $\left(f\left(x_{s}\right), f^{\prime}\left(x_{s}\right)\right) \in C R P_{\mathbf{X}}(\tilde{M})$.

Proof. Again we will use the Levi-Civita covariant derivative $\tilde{\nabla}$ from the embedded metric. First we note that $f^{\prime}\left(x_{s}\right)$ lives in the correct space as $R(f) \subseteq \tilde{M}^{d}$.

To show Inequality 2.39) holds one can use the fact that $\left(f\left(\left(x_{s}\right), f^{\prime}\left(x_{s}\right)\right)\right.$ is a controlled rough path in the embedded space or Taylor's Theorem to see that

$$
f\left(x_{t}\right)-f\left(x_{s}\right)-f^{\prime}\left(x_{s}\right)\left(x_{t}-x_{s}\right) \underset{2}{\approx} 0
$$

which easily implies

$$
P\left(f\left(x_{s}\right)\right)\left[f\left(x_{t}\right)-f\left(x_{s}\right)-f^{\prime}\left(x_{s}\right)\left(x_{t}-x_{s}\right)\right] \underset{2}{\approx} 0 .
$$

But again by Lemma 6.2

$$
\begin{aligned}
& P\left(f\left(x_{s}\right)\right)\left[f\left(x_{t}\right)-f\left(x_{s}\right)-f^{\prime}\left(x_{s}\right)\left(x_{t}-x_{s}\right)\right] \\
& \quad=P\left(f\left(x_{s}\right)\right)\left[f\left(x_{t}\right)-f\left(x_{s}\right)\right]-f^{\prime}\left(x_{s}\right)\left(x_{t}-x_{s}\right) \\
& \quad \approx\left(\exp _{f\left(x_{s}\right)}^{\tilde{\nabla}}\right)^{-1}\left(f\left(x_{t}\right)\right)-f^{\prime}\left(x_{s}\right)\left(x_{t}-x_{s}\right) .
\end{aligned}
$$

Thus

$$
\left(\exp _{f\left(x_{s}\right)}^{\tilde{\nabla}}\right)^{-1}\left(f\left(x_{t}\right)\right)-f^{\prime}\left(x_{s}\right)\left(x_{t}-x_{s}\right) \underset{2}{\approx} 0
$$

Lastly to show Inequality 2.40 , we have

$$
f^{\prime}\left(x_{t}\right)-f^{\prime}\left(x_{s}\right) \underset{1}{\approx} 0
$$

and therefore

$$
\begin{aligned}
0 & \approx P\left(f\left(x_{t}\right)\right)\left[f^{\prime}\left(x_{t}\right)-f^{\prime}\left(x_{s}\right)\right] \\
& =f^{\prime}\left(x_{t}\right)-P\left(f\left(x_{t}\right)\right) f^{\prime}\left(x_{s}\right) \\
& \approx f^{\prime}\left(x_{t}\right)-U_{f\left(x_{t}\right), f\left(x_{s}\right)}^{\tilde{r}} f^{\prime}\left(x_{s}\right),
\end{aligned}
$$

wherein we have used $P\left(f\left(x_{t}\right)\right) f^{\prime}\left(x_{t}\right)=f^{\prime}\left(x_{t}\right)$ in the second line and Lemma6.2 in the last. Thus $\left(f\left(x_{s}\right), f^{\prime}\left(x_{s}\right)\right) \in C R P_{\mathbf{X}}(\tilde{M})$ 


\subsection{Smooth Function Definition of CRP}

In the spirit of semi-martingales on manifolds [see for example [11, Chapter III] or [10, 16, 17]], we can define controlled rough paths on manifolds as elements which, when composed with any smooth function, give rise to a one-dimensional controlled rough path on flat space. More precisely we have the following theorem.

Theorem $2.57 \mathbf{y}=\left(y, y^{\dagger}\right) \in C R P_{\mathbf{X}}(M)$ if and only if for every $f \in C^{\infty}(M)$,

$$
f_{*} \mathbf{y}=\left(f(y), d f \circ y^{\dagger}\right) \in C R P_{\mathbf{X}}(\mathbb{R}) .
$$

Proof. The proof that $\mathbf{y} \in C R P_{\mathbf{X}}(M)$ implies that $f_{*} \mathbf{y} \in C R P_{\mathbf{X}}(\mathbb{R})$ for every $f \in C^{\infty}(M)$ will be deferred to the more general case proved in Proposition 4.10 (in which case we consider the codomain of $f$ to be a manifold $\tilde{M}$ ).

To prove the converse, let $\phi$ be a chart and $0 \leq a<b \leq T$ be such that $y([a, b]) \subseteq D(\phi)$ and let $\mathcal{O} \subset M$ be an open set such that $\overline{\mathcal{O}}$ is compact and

$$
y([a, b]) \subseteq \mathcal{O} \subseteq \overline{\mathcal{O}} \subseteq D(\phi) .
$$

Then by using a cutoff function we can manufacture global functions $f^{i} \in C^{\infty}(M)$ which agree with the coordinates $\phi^{i}$ on $\mathcal{O}$. The assumption that $f_{*}^{i} \mathbf{y} \in C R P_{\mathbf{X}}([a, b], \mathbb{R})$ is a controlled rough path for $1 \leq i \leq d$ then shows the inequalities in Eqs. (2.24) and (2.25) of Definition 2.40 hold.

\section{Integration of Controlled One-Forms}

In the flat case, a controlled rough path with values in an appropriate Euclidean spaces can be integrated against another controlled rough path (see Theorem 2.9p provided their controlling rough path $\mathbf{X}$ is the same. The integral in this case is another rough path controlled by $\mathbf{X}$. We can do something similar on manifolds, though it will be necessary to add some extra structure. As usual let $\mathbf{y}_{s}=\left(y_{s}, y_{s}^{\dagger}\right)$ be a controlled rough path on $M$ controlled by $\mathbf{X}=(x, \mathbb{X}) \in W \oplus W^{\otimes 2}$. Let $V$ be a Banach space.

\subsection{Controlled One-Forms Along a Rough Path}

Let $U$ be a parallelism on $M$.

Definition 3.1 The pair $\left(\alpha_{s}, \alpha_{s}^{\dagger}\right)$ is a $V$-valued $U$-controlled (rough) one-form along $y_{s}$ if

1. $\alpha_{s} \in L\left(T_{y_{s}} M, V\right)$

2. $\alpha_{s}^{\dagger} \in L\left(W \otimes T_{y_{s}} M, V\right)$

3. $\alpha_{t} \circ U\left(y_{t}, y_{s}\right)-\alpha_{s}-\alpha_{s}^{\dagger}\left(x_{s, t} \otimes(\cdot)\right) \underset{2}{\approx} 0$

4. $\alpha_{t}^{\dagger} \circ\left(I \otimes U\left(y_{t}, y_{s}\right)\right)-\alpha_{s}^{\dagger} \underset{1}{\approx} 0$

By items 3 and 4 we mean these hold if $|t-s|<\delta$ for some $\delta>0$ to ensure the expressions make sense. 
Remark 3.2 For the sake of clarity, by item 3 of Definition 3.1, we mean that if $s, t$ are close, then there exists a $C$ such that

$$
\left|\alpha_{t} \circ U\left(y_{t}, y_{s}\right)-\alpha_{s}-\alpha_{s}^{\dagger}\left(x_{s, t} \otimes(\cdot)\right)\right|_{g, o p} \leq C \omega(s, t)^{2 / p} .
$$

For item 4, we mean for $s, t$ close, there exists a $C$ such that

$$
\left|\alpha_{t}^{\dagger} \circ\left(w \otimes U\left(y_{t}, y_{s}\right)\right)-\alpha_{s}^{\dagger}(w \otimes(\cdot))\right|_{g, o p} \leq C|w| \omega(s, t)^{1 / p}
$$

for all $w \in W$. By Corollary 6.4, it does not matter which Riemannian metric $g$ we choose here.

Notation 3.3 Let $C R P_{y}^{U}(M, V)$ denote those $\boldsymbol{\alpha}_{s}:=\left(\alpha_{s}, \alpha_{s}^{\dagger}\right)$ satisfying Definition 3.1. We refer to $C R P_{y}^{U}(M, V)$ as a space of $U$-controlled one-forms along $\mathbf{y}$.

Remark 3.4 If $M=\mathbb{R}^{d}$ and $U=I$ and we identify $T_{y_{s}} M$ with $\mathbb{R}^{d}$ then Definition 3.1 reduces to the flat case definition of a $L\left(\mathbb{R}^{d}, V\right)$ - valued rough path controlled by $\mathbf{X}$.

Remark 3.5 Note that 3 and 4, of Definition 3.1 force continuity of both $\alpha_{s}$ and $\alpha_{s}^{\dagger}$.

We can take linear combinations of elements of $C R P_{y}^{U}(M, V)$ to form other elements in $C R P_{y}^{U}(M, V)$. The following proposition, whose simple proof is left to the reader, shows how to construct more non-trivial examples of elements in $C R P_{y}^{U}(M, V)$.

Proposition 3.6 If $V$ and $\tilde{V}$ are Banach spaces, $\boldsymbol{\alpha} \in C R P_{y}^{U}(M, V)$ and

$$
\mathbf{f}=\left(f, f^{\dagger}\right) \in C R P_{\mathbf{X}}(\operatorname{Hom}(V, \tilde{V}))
$$

then

$$
(\mathbf{f} \boldsymbol{\alpha})_{s}:=\left(f_{s} \alpha_{s}, f_{s}^{\dagger} \alpha_{s}+f_{s} \alpha_{s}^{\dagger}\right) \in C R P_{y}^{U}(M, \tilde{V}) .
$$

where by $f_{s}^{\dagger} \alpha_{s}$ we mean $f_{s}^{\dagger}\left((\cdot) \otimes \alpha_{s}(\cdot)\right)$.

Our next goal is to define an integral of $\boldsymbol{\alpha}_{s}$ along $\mathbf{y}_{s}$. However, this integral will depend on a choice of parallelism and for this reason we need to introduce the "compatibility tensor" which measures the difference between two parallelisms.

\subsection{The Compatibility Tensors}

Definition 3.7 The compatibility tensor, $S^{\tilde{U}, U} \in \Gamma\left(T^{*} M \otimes T^{*} M \otimes T M\right)$, of two parallelisms $\tilde{U}$ and $U$ on $M$ is the defined by

$$
S_{m}^{\tilde{U}, U}:=d\left[U(\cdot, m)^{-1} \tilde{U}(\cdot, m)\right]_{m} .
$$

In more detail if $v_{m}, w_{m} \in T_{m} M$, then

$$
S_{m}^{\tilde{U}, U}\left[v_{m} \otimes w_{m}\right]=v_{m}\left[x \rightarrow U(x, m)^{-1} \tilde{U}(x, m) w_{m}\right] .
$$


Remark 3.8 There are actually multiple ways to define $S_{m}^{\tilde{U}, U}$. For example, we have on simple tensors

$$
\begin{aligned}
S_{m}^{\tilde{U}, U}\left(v_{m} \otimes w_{m}\right) & =d\left[U(m, \cdot) \tilde{U}(m, \cdot)^{-1} w_{m}\right]_{m} v_{m} \\
& =\left(\nabla_{v_{m}}[\tilde{U}(\cdot, m)-U(\cdot, m)]\right) w_{m} \\
& =\left(\nabla_{v_{m}}[U(m, \cdot)-\tilde{U}(m, \cdot)]\right) w_{m}
\end{aligned}
$$

where $\nabla$ is any covariant derivative on $M$. Similar to the proofs of Corollary 2.29 above and Theorem 3.15 below, the identities in Eq. (3.1) are straightforward to prove by employing charts to reduce them to Euclidean space identities.

Example 3.9 If $\nabla$ and $\tilde{\nabla}$ are two covariant derivatives on $T M, U=U^{\nabla}, \tilde{U}=U^{\tilde{\nabla}}$, and $A \in$ $\Omega^{1}($ End $(T M))$ such that $\nabla=\tilde{\nabla}+A$, then

$$
S_{m}^{\tilde{U}, U}\left(v_{m} \otimes w_{m}\right)=A\left(v_{m}\right) w_{m} \in T_{m} M
$$

Indeed,

$$
\begin{aligned}
v_{m}\left[U(\cdot, m)^{-1} \tilde{U}(\cdot, m) w_{m}\right] & =\nabla_{v_{m}}\left[\tilde{U}(\cdot, m) w_{m}\right] \\
& =\tilde{\nabla}_{v_{m}}\left[\tilde{U}(\cdot, m) w_{m}\right]+A\left(v_{m}\right) \tilde{U}(m, m) w_{m} \\
& =0+A\left(v_{m}\right) w_{m}=A\left(v_{m}\right) w_{m}
\end{aligned}
$$

Example 3.10 (Converse of Example 3.9) If $U$ and $\tilde{U}$ are two parallelisms on $M$ and $\nabla=\nabla^{U}$ and $\tilde{\nabla}=\nabla^{\tilde{U}}$ are the corresponding covariant derivatives on $T M$ (as in Remark 2.20), then

$$
\nabla_{v_{m}}=\tilde{\nabla}_{v_{m}}+S_{m}^{\tilde{U}, U}\left(v_{m} \otimes(\cdot)\right) \forall v_{m} \in T_{m} M
$$

The verification is as follows. If $Y$ is a vector-field on $M$ and $\sigma_{t}$ is such that $\dot{o}_{0}=v_{m}$, we have

$$
\begin{aligned}
\nabla_{v_{m}} Y-\tilde{\nabla}_{v_{m}} Y & :=\left.\frac{d}{d t}\right|_{0}\left[U\left(m, \sigma_{t}\right)-\tilde{U}\left(m, \sigma_{t}\right)\right] Y\left(\sigma_{t}\right) \\
& =\left(\nabla_{v_{m}}[U(m, \cdot)-\tilde{U}(m, \cdot)]\right) Y(m)+0 \cdot \nabla_{v_{m}} Y \\
& =S_{m}^{\tilde{U}, U}\left(v_{m} \otimes Y(m)\right)
\end{aligned}
$$

wherein we have used Eq. (3.1) for the last equality.

Lemma 3.11 If $U, \tilde{U}$, and $\hat{U}$ are three parallelisms, then

$$
S^{\hat{U}, U}=S^{\hat{U}, \tilde{U}}+S^{\tilde{U}, U} \text { and } S^{\tilde{U}, U}=-S^{U, \tilde{U}} .
$$

Proof. For $v_{m}, w_{m} \in T_{m} M$, an application of the product rules shows

$$
\begin{aligned}
S_{m}^{\hat{U}, U}\left(v_{m} \otimes w_{m}\right) & =v_{m}\left[U(\cdot, m)^{-1} \hat{U}(\cdot, m) w_{m}\right] \\
& =v_{m}\left[\left[U(\cdot, m)^{-1} \tilde{U}(\cdot, m)\right]\left[\tilde{U}(\cdot, m)^{-1} \hat{U}(\cdot, m)\right] w_{m}\right] \\
& =S_{m}^{\hat{U}, \tilde{U}}\left(v_{m} \otimes w_{m}\right)+S_{m}^{\tilde{U}, U}\left(v_{m} \otimes w_{m}\right) .
\end{aligned}
$$


Similarly,

$$
\begin{aligned}
S^{U, \tilde{U}}\left[v_{m} \otimes(\cdot)\right] & =v_{m}\left[\tilde{U}(\cdot, m)^{-1} U(\cdot, m)\right] \\
& =v_{m}\left[U(\cdot, m)^{-1} \tilde{U}(\cdot, m)\right]^{-1} \\
& =-v_{m}\left[U(\cdot, m)^{-1} \tilde{U}(\cdot, m)\right] \\
& =-S^{\tilde{U}, U}\left[v_{m} \otimes(\cdot)\right] .
\end{aligned}
$$

Notation 3.12 If $\mathcal{G}:=(\psi, U)$ is a gauge, we let $S^{\mathcal{G}}:=S^{\psi_{*}, U}$ be the compatibility tensor between $U^{\psi}$ and $U$, where $U^{\psi}(m, n):=\psi(m, \cdot)_{* n}$ as in Remark 2.21.

If we have a covariant derivative $\nabla$ on $M$, then as in Example 2.19 we have the choice of gauge $\mathcal{G}=(\psi, U)=\left(\left(\exp ^{\nabla}\right)^{-1}, U^{\nabla}\right)$. In this case, the tensor $S_{m}^{\mathcal{G}}$ is a more familiar object.

Lemma 3.13 If $\psi=\left(\exp ^{\nabla}\right)^{-1}$ and $U=U^{\nabla}$, then

$$
S_{m}^{\mathcal{G}}=\frac{1}{2} T_{m}^{\nabla}
$$

where $T^{\nabla}$ is the Torsion tensor of $\nabla$.

Proof. By transferring the covariant derivative and functions using charts, we may assume we are working on Euclidean space. In this case, by Eq. 6.10 and Corollary 6.6, we have

$$
\begin{aligned}
S_{m}^{\mathcal{G}}((m, v) \otimes(m, w)) & =\left(\nabla_{(m, v)}\left[U_{m, \cdot}^{\nabla}-\left(\exp _{m}^{\nabla}\right)_{* \cdot}^{-1}\right]\right) w \\
& =\left[\partial_{(m, v)}+A_{m}\langle v\rangle\right]\left[U_{m, \cdot}^{\nabla}-\left(\exp _{m}^{\nabla}\right)_{* \cdot}^{-1}\right] w \\
& =\left(U_{m, \cdot}^{\nabla}\right)^{\prime}(m)[v \otimes w]-\left(\left(\exp _{m}^{\nabla}\right)^{-1}\right)^{\prime \prime}(m)[v \otimes w]+A_{m}\langle v\rangle\langle w\rangle-A_{m}\langle v\rangle\langle w\rangle \\
& =A_{m}\langle v\rangle\langle w\rangle-\frac{1}{2} A_{m}\langle v\rangle\langle w\rangle-\frac{1}{2} A_{m}\langle w\rangle\langle v\rangle \\
& =\frac{1}{2}\left[A_{m}\langle v\rangle\langle w\rangle-A_{m}\langle w\rangle\langle v\rangle\right] \\
& =\frac{1}{2} T_{m}^{\nabla}((m, v) \otimes(m, w)) .
\end{aligned}
$$

Here is one last example of a gauge and its compatibility tensor.

Proposition 3.14 Let $G$ be a Lie group and $\nabla$ be the left covariant derivative on TG uniquely determined by requiring the left invariant vector fields to be covariantly constant, i.e. $\nabla \tilde{A}=0$ for all $A \in \mathfrak{g}$. Then for $g$ near $k$,

$$
U^{\nabla}(g, k)=/ /(k \rightarrow g)=L_{g k^{-1} *},
$$


and

$$
\psi^{\nabla}(k, g)=\left(\exp _{k}^{\nabla}\right)^{-1}(g)=k \cdot \log \left(k^{-1} g\right)
$$

where $L_{g}: G \rightarrow G$ is left multiplication by $g \in G$ and $\log$ is the local inverse of the map $A \rightarrow e^{A}$. Moreover the compatibility tensor for this gauge is given by

$$
S\left(\xi_{g}, \eta_{g}\right)=-\frac{1}{2} L_{g *}\left[\theta\left(\xi_{g}\right), \theta\left(\eta_{g}\right)\right] \text { for all } \xi_{g}, \eta_{g} \in T_{g} G
$$

where $\theta$ is the Maurer-Cartan form on $G$ defined by $\theta(\xi):=L_{g^{-1}} \xi \in \mathfrak{g}:=T_{e} G$ for all $\xi \in T_{g} G$.

Proof. The torsion of $\nabla$ is given by

$$
T(\tilde{A}, \tilde{B})=\nabla_{\tilde{A}} \tilde{B}-\nabla_{\tilde{B}} \tilde{A}-[\tilde{A}, \tilde{B}]=-\widetilde{[A, B]}
$$

or equivalently as

$$
T\left(\xi_{g}, \eta_{g}\right)=-L_{g *}\left[\theta\left(\xi_{g}\right), \theta\left(\eta_{g}\right)\right] \text { for all } \xi_{g}, \eta_{g} \in T_{g} G .
$$

Eq. (3.4) follows from the above formula along with the result in Lemma 3.13

If $\xi(t)$ is a path $T G$ above $\sigma(t) \in G$ it may be written as $\xi(t)=L_{\sigma(t) *} \theta(\xi(t))$. Since $L_{\sigma(t) *}$ is parallel translation, it follows that

$$
\frac{\nabla \xi(t)}{d t}=L_{\sigma(t) *} \frac{d}{d t} \theta(\xi(t))
$$

Thus $\xi(t) \in T G$ is parallel iff $\theta(\xi(t))$ is constant for all $t$. If $\sigma$ is a general curve in $G$, we may conclude

$$
/ /\left(\left.\sigma\right|_{[s, t]}\right)=L_{\sigma(t) *} L_{\sigma(s)^{-1} *}=L_{\sigma(t) \sigma(s)^{-1} *}
$$

and therefore $U^{\nabla}$ is given as in Eq. 3.2 .

A curve $\sigma(t) \in G$ is a geodesic iff $\dot{\sigma}(t)$ is parallel iff $\theta(\dot{\sigma}(t))=A$ for some $A \in \mathfrak{g}$. That is $\dot{\sigma}(t)=\tilde{A}(\sigma(t))$ with $\sigma(0)=k \in G$. The solution to this equation is $\sigma(t)=k e^{t A}$ and hence we have shown that $\exp _{k}^{\nabla}(k \cdot A)=k e^{A}$. So setting $g=k e^{A}$ and solving for $A$ gives $A=\log \left(k^{-1} g\right)$ and the formula for $\psi^{\nabla}$ in Eq. 3.3. now follows.

The last three results of this subsection show how the compatibility tensor allows us to compare two different parallelisms and two different logarithms on $M$.

Theorem 3.15 Suppose that $U$ and $\tilde{U}$ are two parallelisms on $M$ and $\psi$ is a logarithm on $M$, then

$$
U(m, n) \tilde{U}(m, n)^{-1}={ }_{2} I+S_{m}^{\tilde{U}, U}(\psi(m, n) \otimes(\cdot)) .
$$

Proof. By using charts it suffices to prove the theorem when $M=\mathbb{R}^{d}$. By Taylor's theorem (see Theorem 2.24,

$$
\begin{aligned}
& U(m, n)={ }_{2} I+\left[\left(D_{2} U\right)(m, m)(n-m)\right] \text { and } \\
& \tilde{U}(m, n)={ }_{2} I+\left[\left(D_{2} \tilde{U}\right)(m, m)(n-m)\right]
\end{aligned}
$$

and therefore

$$
\begin{aligned}
U(m, n) \tilde{U}(m, n)^{-1} & ={ }_{2}\left(I+\left[\left(D_{2} U\right)(m, m)(n-m)\right]\right)\left(I-\left[\left(D_{2} \tilde{U}\right)(m, m)(n-m)\right]\right) \\
& ={ }_{2} I+\left[\left(\left(D_{2} U\right)(m, m)-\left(D_{2} \tilde{U}\right)(m, m)\right)(n-m)\right] .
\end{aligned}
$$


However, by Eq. (3.1) we have

$$
S_{m}^{\tilde{U}, U}=\left(D_{2} U\right)(m, m)-\left(D_{2} \tilde{U}\right)(m, m) .
$$

Using this identity back in Eq. (3.6) shows

$$
U(m, n) \tilde{U}(m, n)^{-1}={ }_{2} I+S_{m}^{\tilde{U}, U}\left([n-m]_{m} \otimes(\cdot)\right)
$$

from which Eq. 3.5 follows because $\psi(m, n)=_{2}[n-m]_{m}$.

Corollary 3.16 If $\mathcal{G}=(\psi, U)$ is a gauge on $M$, then

$$
\psi(n, \cdot)_{* m}={ }_{2} U(n, m)\left[I+S_{m}^{\mathcal{G}}(\psi(m, n) \otimes(\cdot))\right] .
$$

In particular

$$
\psi\left(y_{t}, \cdot\right)_{* y_{s}} \approx U\left(y_{t}, y_{s}\right)\left[I+S_{y_{s}}^{\mathcal{G}}\left(\psi\left(y_{s}, y_{t}\right) \otimes(\cdot)\right)\right] .
$$

Proof. Theorem 3.15 implies

$$
U(m, n) \psi(m, \cdot)_{* n}^{-1}={ }_{2} I+S_{m}^{\mathcal{G}}(\psi(m, n) \otimes(\cdot))
$$

while Corollary 2.29 shows,

$$
U(m, n)^{-1}={ }_{2} U(n, m) \text { and } \psi(m, \cdot)_{* n}^{-1}={ }_{2} \psi(n, \cdot)_{* m} .
$$

Eq. 3.8 now easily follows from the last two displayed equations. The second statement follows by patching.

Lastly we may use the compatibility tensor to compare two logarithms.

Proposition 3.17 Suppose that $\psi$ and $\tilde{\psi}$ are two logarithms on a manifold $M$. Then the compatibility tensor, $S^{\psi_{*}, \tilde{\psi}_{*}}$ is symmetric and

$$
\psi(m, n)-\tilde{\psi}(m, n)={ }_{3} \frac{1}{2} S_{m}^{\tilde{\psi}_{*}, \psi_{*},}(\psi(m, n) \otimes \psi(m, n)) .
$$

Proof. As usual it suffices to prove this result when $M=\mathbb{R}^{d}$ in which case we omit the base points of tangent vectors. From Eq. 3.7 with $U(x, y)=\psi_{x}^{\prime}(y)$ and $\tilde{U}(x, y)=\tilde{\psi}_{x}^{\prime}(y)$, we see that

$$
S_{x}^{\tilde{\psi}_{*}, \psi_{*}}=\psi_{x}^{\prime \prime}(x)-\tilde{\psi}_{x}^{\prime \prime}(x)
$$

which is symmetric since mixed partial derivatives commute. Then by Taylor's theorem and Eq. (3.11,

$$
\begin{aligned}
\psi(x, y)-\tilde{\psi}(x, y) & =\frac{1}{2}\left[\psi_{x}^{\prime \prime}(x)-\tilde{\psi}_{x}^{\prime \prime}(x)\right](y-x)^{\otimes 2}+O\left(|y-x|^{3}\right) \\
& =\frac{1}{2} S_{x}^{\tilde{\psi}_{*}, \psi_{*}}\left(\psi(x, y)^{\otimes 2}\right)+O\left(|y-x|^{3}\right)
\end{aligned}
$$

wherein we have also used $(y-x)^{\otimes 2}={ }_{3} \psi(x, y)^{\otimes 2}$.

Remark 3.18 If $\nabla$ is any covariant derivative on $T M$, then

$$
S_{m}^{\tilde{\psi}_{*}, \psi_{*}}=[\nabla d(\psi(m, \cdot)-\tilde{\psi}(m, \cdot))]_{m}=\operatorname{Hess}_{m}^{\nabla}\left(\psi_{m}-\tilde{\psi}_{m}\right)
$$

where $\operatorname{Hess}_{m}^{\nabla} f:=[\nabla d f]_{m}$. By choosing $\nabla$ to be Torsion free we again see that $S_{m}^{\tilde{\psi}_{*}, \psi_{*}}$ is a symmetric tensor. 


\section{3 $\quad U$ - Controlled Rough Integration}

Our next goal is to construct "the" integral, $\int\langle\boldsymbol{\alpha}, d \mathbf{y}\rangle$, where $\mathbf{y} \in C R P_{\mathbf{X}}(M)$ and $\boldsymbol{\alpha} \in C R P_{y}^{U}(M, V)$. We begin with the following proposition in the smooth category which is meant to motivate the definitions to come.

Proposition 3.19 Assume (in this proposition only) that all functions, $\mathbf{y}_{s}, \boldsymbol{\alpha}_{s}$, and $x_{s}$ are smooth, $p=1$, and $\omega(s, t)=|t-s|$. Further assume $\mathbf{y}$ (respectively $\boldsymbol{\alpha}$ ) still satisfy the estimates of being controlled rough path (along $\mathbf{y})$. Then

$$
\int_{s}^{t} \alpha_{\tau} \dot{y}_{\tau} d \tau=\alpha_{s}\left[\psi\left(y_{s}, y_{t}\right)+S_{y_{s}}^{\mathcal{G}}\left(y_{s}^{\dagger} \otimes y_{s}^{\dagger} \mathbb{X}_{s, t}\right)\right]+\alpha_{s}^{\dagger}\left(I \otimes y_{s}^{\dagger}\right) \mathbb{X}_{s, t}+O\left((t-s)^{3}\right) .
$$

Proof. Our assumptions give,

$$
\begin{aligned}
& \psi\left(y_{s}, y_{t}\right)=y_{s}^{\dagger} x_{s, t}+O\left((t-s)^{2}\right) \Longrightarrow \dot{y}_{s}=y_{s}^{\dagger} \dot{x}_{s}, \\
& \alpha_{t} U\left(y_{t}, y_{s}\right)=\alpha_{s}+\alpha_{s}^{\dagger} x_{s, t}+O\left((t-s)^{2}\right), \\
& U\left(y_{s}, y_{t}\right) y_{t}^{\dagger}=y_{s}+O(t-s), \text { and } \\
& \alpha_{t}^{\dagger}\left(I \otimes U\left(y_{t}, y_{s}\right)\right)=\alpha_{s}^{\dagger}+O(t-s) .
\end{aligned}
$$

We start with the identity,

$$
\begin{aligned}
\int_{s}^{t} \alpha_{\tau} \dot{y}_{\tau} d \tau & =\int_{s}^{t} \alpha_{\tau} U\left(y_{\tau}, y_{s}\right) U\left(y_{\tau}, y_{s}\right)^{-1} \dot{y}_{\tau} d \tau \\
& =\int_{s}^{t}\left[\alpha_{s}+\alpha_{s}^{\dagger} x_{s, \tau}+O\left((\tau-s)^{2}\right)\right] U\left(y_{\tau}, y_{s}\right)^{-1} \dot{y}_{\tau} d \tau \\
& =\int_{s}^{t} \alpha_{s} U\left(y_{\tau}, y_{s}\right)^{-1} \dot{y}_{\tau} d \tau+\int_{s}^{t} \alpha_{s}^{\dagger} x_{s, \tau} U\left(y_{\tau}, y_{s}\right)^{-1} \dot{y}_{\tau} d \tau+O\left((t-s)^{3}\right) \\
& =\int_{s}^{t} \alpha_{s} U\left(y_{s}, y_{\tau}\right) \dot{y}_{\tau} d \tau+\int_{s}^{t} \alpha_{s}^{\dagger} x_{s, \tau} U\left(y_{s}, y_{\tau}\right) \dot{y}_{\tau} d \tau+O\left((t-s)^{3}\right) . \\
& =: A+B+O\left((t-s)^{3}\right)
\end{aligned}
$$

wherein we have used Corollary 2.29 in order to show it is permissible to replace $U\left(y_{\tau}, y_{s}\right)^{-1}$ by $U\left(y_{s}, y_{\tau}\right)$ above. The $B$ term is then easily estimated as

$$
\begin{aligned}
B & =\int_{s}^{t} \alpha_{s}^{\dagger} x_{s, \tau} U\left(y_{s}, y_{\tau}\right) \dot{y}_{\tau} d \tau=\int_{s}^{t} \alpha_{s}^{\dagger} x_{s, \tau} U\left(y_{s}, y_{\tau}\right) y_{\tau}^{\dagger} \dot{x}_{\tau} d \tau \\
& =\int_{s}^{t} \alpha_{s}^{\dagger} x_{s, \tau} y_{s}^{\dagger} \dot{x}_{\tau} d \tau+O\left((t-s)^{3}\right)=\alpha_{s}^{\dagger}\left(I \otimes y_{s}^{\dagger}\right) \mathbb{X}_{s, t}+O\left((t-s)^{3}\right) .
\end{aligned}
$$

The estimate of the $A$ term to order $O\left((t-s)^{3}\right)$ requires more care. For this term we use

$$
\frac{d}{d t} \psi\left(y_{s}, y_{t}\right)=\psi\left(y_{s}, \cdot\right)_{* y_{t}} \dot{y}_{t} \Longrightarrow \dot{y}_{t}=\psi\left(y_{s}, \cdot\right)_{* y_{t}}^{-1} \frac{d}{d t} \psi\left(y_{s}, y_{t}\right)
$$


and (from Theorem 3.15) that

$$
U\left(y_{s}, y_{\tau}\right) \psi\left(y_{s}, \cdot\right)_{* y_{\tau}}^{-1}={ }_{2} I+S_{y_{s}}^{\mathcal{G}}\left(\psi\left(y_{s}, y_{\tau}\right) \otimes(\cdot)\right)
$$

in order to conclude,

$$
\begin{aligned}
A & :=\int_{s}^{t} \alpha_{s} U\left(y_{s}, y_{\tau}\right) \dot{y}_{\tau} d \tau=\int_{s}^{t} \alpha_{s} U\left(y_{s}, y_{\tau}\right) \psi\left(y_{s}, \cdot\right)_{* y_{\tau}}^{-1} \frac{d}{d \tau} \psi\left(y_{s}, y_{\tau}\right) d \tau \\
& =\int_{s}^{t} \alpha_{s}\left[I+S_{y_{s}}^{\mathcal{G}}\left(\psi\left(y_{s}, y_{\tau}\right) \otimes(\cdot)\right)\right] \frac{d}{d \tau} \psi\left(y_{s}, y_{\tau}\right) d \tau+O\left(|t-s|^{3}\right) \\
& =\alpha_{s}\left(\psi\left(y_{s}, y_{t}\right)\right)+\alpha_{s} \int_{s}^{t} S_{y_{s}}^{\mathcal{G}}\left(\psi\left(y_{s}, y_{\tau}\right) \otimes \frac{d}{d \tau} \psi\left(y_{s}, y_{\tau}\right)\right) d \tau+O\left(|t-s|^{3}\right) \\
& =\alpha_{s}\left(\psi\left(y_{s}, y_{t}\right)\right)+\alpha_{s} \int_{s}^{t} S_{y_{s}}^{\mathcal{G}}\left(y_{s}^{\dagger} x_{s, \tau} \otimes y_{s}^{\dagger} \dot{x}_{\tau}\right) d \tau+O\left(|t-s|^{3}\right) \\
& =\alpha_{s}\left(\psi\left(y_{s}, y_{t}\right)\right)+\alpha_{s} S_{y_{s}}^{\mathcal{G}}\left(y_{s}^{\dagger} \otimes y_{s}^{\dagger} \mathbb{X}_{s, t}\right)+O\left(|t-s|^{3}\right)
\end{aligned}
$$

Putting this all together proves Eq. 3.12.

The following definition is motivated by the right hand side of Eq. 3.12.

Definition $3.20((\mathcal{G}, \mathbf{y})$ - integrator $)$ Given a gauge $\mathcal{G}:=(\psi, U)$ and $\mathbf{y} \in C R P_{\mathbf{X}}(M)$, the $(\mathcal{G}, \mathbf{y})-$ integrator is the increment process;

$$
\mathbf{y}_{s, t}^{\mathcal{G}}:=\left(\psi\left(y_{s}, y_{t}\right)+S_{y_{s}}^{\mathcal{G}}\left(y_{s}^{\dagger \otimes 2} \mathbb{X}_{s, t}\right),\left(I \otimes y_{s}^{\dagger}\right) \mathbb{X}_{s, t}\right) \in T_{y_{s}} M \times\left[W \otimes T_{y_{s}} M\right] .
$$

Moreover, for $\boldsymbol{\alpha} \in C R P_{y}^{U}(M, V)$ (see Notation 3.3) let

$$
\tilde{z}_{s, t}:=\left\langle\boldsymbol{\alpha}_{s}, \mathbf{y}_{s, t}^{\mathcal{G}}\right\rangle=\alpha_{s}\left(\psi\left(y_{s}, y_{t}\right)+S_{y_{s}}^{\mathcal{G}}\left(y_{s}^{\dagger \otimes 2} \mathbb{X}_{s, t}\right)\right)+\alpha_{s}^{\dagger}\left(I \otimes y_{s}^{\dagger}\right) \mathbb{X}_{s, t}
$$

which is defined for $(s, t) \in \Delta_{[0, T]}$ with $|t-s|<\delta$ for some sufficiently small $\delta>0$.

Recall that a two-parameter function $F: \Delta_{[0, T]} \longrightarrow V$ is an almost additive functional if there exists a $\theta>1$, a control $\tilde{\omega}(s, t)$ and a $C>0$ such that

$$
\left|F_{s, u}-F_{s, t}-F_{t, u}\right| \leq C \tilde{\omega}(s, t)^{\theta}
$$

for all $0 \leq s \leq t \leq u \leq T$.

Theorem 3.21 Let $\mathcal{G}:=(\psi, U)$ be a gauge, $\boldsymbol{\alpha} \in C R P_{y}^{U}(M, V)$, and $\tilde{z}_{s, t}$ be as in Definition 3.20. Then there exists a unique $\mathbf{z}=\left(z, z^{\dagger}\right) \in C R P_{\mathbf{X}}(V)$ such that $z_{0}=0, z_{s, t} \underset{3}{\approx} \tilde{z}_{s, t}$, and $z_{s}^{\dagger}=\alpha_{s} \circ y_{s}^{\dagger}$. We denote this unique controlled rough path by $\int\left\langle\boldsymbol{\alpha}, d \mathbf{y}^{\mathcal{G}}\right\rangle$, i.e.

$$
\int_{s}^{t}\left\langle\boldsymbol{\alpha}, d \mathbf{y}^{\mathcal{G}}\right\rangle:=\left[\int\left\langle\boldsymbol{\alpha}, d \mathbf{y}^{\mathcal{G}}\right\rangle\right]_{s, t}^{1} \underset{3}{\approx}\left\langle\boldsymbol{\alpha}_{s}, \mathbf{y}_{s, t}^{\mathcal{G}}\right\rangle \text { and }\left[\int\left\langle\boldsymbol{\alpha}, d \mathbf{y}^{\mathcal{G}}\right\rangle\right]_{s}^{\dagger}=\alpha_{s} \circ y_{s}^{\dagger} .
$$


Proof. By Theorem 3.26 below, $\tilde{z}_{s, t}:=\left\langle\boldsymbol{\alpha}_{s}, \mathbf{y}_{s, t}^{\mathcal{G}}\right\rangle$ is an almost additive functional and therefore by Lyons 22. Theorem 3.3.1] there exists a unique additive functional $z_{s, t}$ such that $z_{s, t} \approx \tilde{z}_{s, t}$. Moreover,

$$
z_{s, t} \underset{3}{\approx} \tilde{z}_{s, t} \underset{2}{\approx} \alpha_{s}\left(\psi\left(y_{s}, y_{t}\right)\right) \underset{2}{\approx} \alpha_{s}\left(y_{s}^{\dagger} x_{s, t}\right)
$$

which shows that $\mathbf{z}_{s}:=\left(z_{s}, \alpha_{s} \circ y_{s}^{\dagger}\right)$ is indeed a controlled rough path with values in $V$.

Example 3.22 In the case that $U=U^{\psi}$ so that

$$
\alpha_{t} \circ\left(\psi_{y_{t}}\right)_{* y_{s}}-\alpha_{s}-\alpha_{s}^{\dagger}\left(x_{s, t} \otimes(\cdot)\right) \underset{2}{\approx} 0
$$

we have that $\mathbf{y}_{s, t}^{\mathcal{G}}:=\left(\psi\left(y_{s}, y_{t}\right),\left(I \otimes y_{s}^{\dagger}\right) \mathbb{X}_{s, t}\right)$ and so

$$
\int_{s}^{t}\left\langle\boldsymbol{\alpha}, d \mathbf{y}^{\mathcal{G}^{\psi}}\right\rangle \underset{3}{\approx} \alpha_{s}\left(\psi\left(y_{s}, y_{t}\right)\right)+\alpha_{s}^{\dagger}\left(I \otimes y_{s}^{\dagger}\right) \mathbb{X}_{s, t} .
$$

Example 3.23 If $\mathcal{G}^{\nabla}=\left(\left(\exp ^{\nabla}\right)^{-1}, U^{\nabla}\right)$, then by Lemma 3.13, we have that

$$
\int_{s}^{t}\left\langle\boldsymbol{\alpha}, d \mathbf{y}^{\mathcal{G}^{\nabla}}\right\rangle \underset{3}{\approx} \alpha_{s}\left(\exp _{y_{s}}^{-1}\left(y_{t}\right)\right)+\alpha_{s}^{\dagger}\left(I \otimes y_{s}^{\dagger}\right) \mathbb{X}_{s, t}+\alpha_{s}\left(\frac{1}{2} T_{y_{s}}^{\nabla} \circ y_{s}^{\dagger \otimes 2} \mathbb{X}_{s, t}\right)
$$

If $\mathbf{f}, \boldsymbol{\alpha}$, and $\mathbf{f} \boldsymbol{\alpha} \in C R P_{y}^{U}(M, \tilde{V})$ are as in Proposition 3.6 then the following expected associativity property holds.

Theorem 3.24 (Associativity Theorem I) Let us continue the notation in Theorem 3.21. If $\mathbf{f}$ and $\mathbf{f} \boldsymbol{\alpha}:=\left(f_{s} \alpha_{s}, f_{s}^{\dagger}\left(I \otimes \alpha_{s}\right)+f_{s} \alpha_{s}^{\dagger}\right)$ are as in Proposition 3.6 and $\mathbf{z}=\left(z, z^{\dagger}\right)=\int\left\langle\boldsymbol{\alpha}, d \mathbf{y}^{\mathcal{G}}\right\rangle$, then

$$
\int\langle\mathbf{f}, d \mathbf{z}\rangle=\int\left\langle\mathbf{f} \boldsymbol{\alpha}, d \mathbf{y}^{\mathcal{G}}\right\rangle
$$

or in other words,

$$
\int\left\langle\mathbf{f}, d \int\left\langle\boldsymbol{\alpha}, d \mathbf{y}^{\mathcal{G}}\right\rangle\right\rangle=\int\left\langle\mathbf{f} \boldsymbol{\alpha}, d \mathbf{y}^{\mathcal{G}}\right\rangle
$$

Proof. We have the approximations

$$
\begin{aligned}
& {\left[\int\left\langle\mathbf{f} \boldsymbol{\alpha}, d \mathbf{y}^{\mathcal{G}}\right\rangle\right]_{s, t}^{1} \underset{3}{\approx} f_{s} \alpha_{s}\left(\psi\left(y_{s}, y_{t}\right)+\mathcal{S}_{y_{s}}^{\mathcal{G}}\left(y_{s}^{\dagger \otimes 2} \mathbb{X}_{s, t}\right)\right)+\left[\left(f_{s}^{\dagger}\left(I \otimes \alpha_{s}\right)+f_{s} \alpha_{s}^{\dagger}\right)\right]\left(I \otimes y_{s}^{\dagger}\right) \mathbb{X}_{s, t}} \\
& =f_{s}\left(\alpha_{s}\left(\psi\left(y_{s}, y_{t}\right)+\mathcal{S}_{y_{s}}^{\mathcal{G}}\left(y_{s}^{\dagger \otimes 2} \mathbb{X}_{s, t}\right)\right)+\alpha_{s}^{\dagger}\left(I \otimes y_{s}^{\dagger}\right) \mathbb{X}_{s, t}\right)+f_{s}^{\dagger}\left(I \otimes \alpha_{s} y_{s}^{\dagger}\right) \mathbb{X}_{s, t} \\
& \underset{3}{\approx} f_{s}\left(z_{s, t}\right)+f_{s}^{\dagger}\left(I \otimes z_{s}^{\dagger}\right) \mathbb{X}_{s, t} \\
& \approx\left[\int_{3}[\mathbf{f}, d \mathbf{z}\rangle\right]_{s, t}^{1}
\end{aligned}
$$

As the first and last terms of this equation are additive functionals, they must be equal.

Secondly

$$
\left[\int\left\langle\mathbf{f} \boldsymbol{\alpha}, d \mathbf{y}^{\mathcal{G}}\right\rangle\right]_{s}^{\dagger}=f_{s} \alpha_{s}\left(y_{s}^{\dagger}\right)=f_{s} z_{s}^{\dagger}=\left[\int\langle\mathbf{f}, d \mathbf{z}\rangle\right]_{s}^{\dagger} .
$$

Thus, the two controlled rough paths are equal. 
Remark 3.25 The $(\mathcal{G}, \mathbf{y})$ - integrator $\mathbf{y}_{s, t}^{\mathcal{G}}$ is helpful in easing notation so that the integral is simply written $\int_{s}^{t}\left\langle\boldsymbol{\alpha}, d \mathbf{y}^{\mathcal{G}}\right\rangle$. A more honest notation for this integral would be

$$
\int_{\mathcal{s}}^{t}\left\langle\left(\alpha, \alpha^{\dagger}\right), d\left(y^{\psi}, \mathbb{X}\right)\right\rangle_{\mathcal{S}_{y^{\dagger}}^{\mathcal{G}}}
$$

where $\mathcal{S}_{y^{\dagger}}^{\mathcal{G}}(s)$ is the block matrix defined by

$$
\mathcal{S}_{y^{\dagger}}^{\mathcal{G}}(s):=\left(\begin{array}{cc}
I & S_{y_{s}}^{\mathcal{G}} \circ\left(y_{s}^{\dagger}\right)^{\otimes 2} \\
0 & I \otimes y_{s}^{\dagger}
\end{array}\right)
$$

and $\langle\cdot, \cdot\rangle_{\mathcal{S}_{y^{\dagger}}^{\mathcal{G}}}$ is the "inner product" given by the matrix $\mathcal{S}_{y^{\dagger}}^{\mathcal{G}}$. When $s$ is close to $t$, we have

$$
\begin{aligned}
\int_{s}^{t}\left\langle\left(\alpha, \alpha^{\dagger}\right), d\left(y^{\psi}, \mathbb{X}\right)\right\rangle_{\mathcal{S}_{y^{\dagger}}^{\mathcal{G}}} \approx\left(\alpha_{s}, \alpha_{s}^{\dagger}\right)\left(\begin{array}{cc}
I & S_{y_{s}}^{\mathcal{G}} \circ\left(y_{s}^{\dagger}\right)^{\otimes 2} \\
0 & I \otimes y_{s}^{\dagger}
\end{array}\right)\left(\begin{array}{c}
y_{s, t}^{\psi} \\
\mathbb{X}_{s, t}
\end{array}\right) \\
=\alpha_{s}\left(\psi\left(y_{s}, y_{t}\right)+S_{y_{s}}^{\mathcal{G}}\left(y_{s}^{\dagger \otimes 2} \mathbb{X}_{s, t}\right)\right)+\alpha_{s}^{\dagger}\left(I \otimes y_{s}^{\dagger}\right) \mathbb{X}_{s, t}
\end{aligned}
$$

\subsection{Almost Additivity Result}

The following theorem was the key ingredient in the proof of Theorem 3.21 on the existence of rough path integration in the manifold setting.

Theorem 3.26 (Almost Additivity) If $\mathcal{G}:=(\psi, U)$ is a gauge and $\boldsymbol{\alpha} \in C R P_{y}^{U}(M, V)$, then $\tilde{z}_{s, t} \in V$ defined as in Definition 3.20 is an almost additive functional.

The proof of Theorem 3.26 will be given after Corollary 3.29 which states that logarithms are "almost additive." We first need a couple of lemmas. Recall from Definition 2.15 that $\psi_{x}=\psi(x, \cdot)$.

Lemma 3.27 If $U, \tilde{U}$ are two parallelisms on $M$, then

$$
S_{y_{t}}^{\tilde{U}, U} \circ U\left(y_{t}, y_{s}\right)^{\otimes 2} \approx U\left(y_{t}, y_{s}\right) \circ S_{y_{s}}^{\tilde{U}, U}
$$

Proof. By the usual patching arguments it suffices to prove this lemma for $M=\mathbb{R}^{d}$. In the Euclidean space setting the identity is trivial to prove since $U(n, m)={ }_{1} I$ and $S_{n}^{\tilde{U}, U}={ }_{1} S_{m}^{\tilde{U}, U}$.

Lemma 3.28 Let $K$ be a compact, convex set in $\mathbb{R}^{d}$. If $\psi$ is a logarithm with domain $\mathcal{D}$ and $K \times K \subseteq \mathcal{D}$, then there exists a $C_{K}$ such that

$$
\left|\psi_{y}^{\prime}(x) \psi(x, y)+\psi(y, z)-\psi_{y}^{\prime}(x) \psi(x, z)\right| \leq C_{K} \max \{|\psi(x, y)|,|\psi(y, z)|,|\psi(x, z)|\}^{3}
$$

for all $x, y, z \in K$.

Proof. We will use the notation $|x, y, z|:=\max \{|y-x|,|z-y|,|z-x|\}$ and write $f(x, y, z)={ }_{k}$ $g(x, y, z)$ iff $f(x, y, z)=g(x, y, z)+O\left(|x, y, z|^{k}\right)$. Since $\psi$ is zero on the diagonal and $\psi_{y}^{\prime}(y)=i d$ 
for all $y$, it follow from Taylor's theorem (or see Theorem 2.24) that

$$
\begin{aligned}
\psi_{y}^{\prime}(x) & ={ }_{2} i d+\psi_{y}^{\prime \prime}(y)(x-y) \text { and } \\
\psi(x, y) & ={ }_{3}(y-x)+\frac{1}{2} \psi_{x}^{\prime \prime}(x)(y-x)^{\otimes 2} \\
& ={ }_{3}(y-x)+\frac{1}{2} \psi_{y}^{\prime \prime}(y)(y-x)^{\otimes 2} .
\end{aligned}
$$

from these approximations we learn,

$$
\psi(x, y)-\psi(x, z)={ }_{3} y-z+\frac{1}{2} \psi_{y}^{\prime \prime}(y)\left[(y-x)^{\otimes 2}-(z-x)^{\otimes 2}\right]
$$

and

$$
\begin{aligned}
\psi_{y}^{\prime}(x) \psi(x, y) & -\psi_{y}^{\prime}(x) \psi(x, z) \\
& ={ }_{3}\left[i d+\psi_{y}^{\prime \prime}(y)(x-y) \otimes(\cdot)\right](\psi(x, y)-\psi(x, z)) \\
& ={ }_{3} y-z+\frac{1}{2} \psi_{y}^{\prime \prime}(y)\left[(y-x)^{\otimes 2}-(z-x)^{\otimes 2}\right]+\psi_{y}^{\prime \prime}(y)[(x-y) \otimes(y-z)] .
\end{aligned}
$$

As simple calculation now shows, with $a=y-x$ and $b=y-z$, that

$$
\frac{1}{2}\left[(y-x)^{\otimes 2}-(z-x)^{\otimes 2}\right]+(x-y) \otimes(y-z)=-\frac{1}{2}\left[b^{\otimes 2}+b \otimes a-a \otimes b\right] .
$$

Since $\psi_{y}^{\prime \prime}(y) a \otimes b=\psi_{y}^{\prime \prime}(y) b \otimes a$ (mixed partial derivatives commute), the last two displayed equations give

$$
\begin{aligned}
\psi_{y}^{\prime}(x) \psi(x, y)-\psi_{y}^{\prime}(x) \psi(x, z) & ={ }_{3} y-z-\frac{1}{2} \psi_{y}^{\prime \prime}(y) b^{\otimes 2} \\
& =-\left[(z-y)+\frac{1}{2} \psi_{y}^{\prime \prime}(y)(z-y)^{\otimes 2}\right]={ }_{3}-\psi(y, z) .
\end{aligned}
$$

The bounds derived above are uniform over a compact set $K$. Because of Eq. (3.17), we may replace $O\left(|x, y, z|^{3}\right)$ with $O\left(\max \{|\psi(x, y)|,|\psi(y, z)|,|\psi(x, z)|\}^{3}\right)$.

Corollary 3.29 If $\left(y_{s}, y_{s}^{\dagger}\right)$ is a controlled rough path and $\psi$ is a logarithm, there exists $C_{\psi}, \delta_{\psi}>0$ such that if $0 \leq s \leq t \leq u \leq T$ and $u-s \leq \delta_{\psi}$, then

$$
\left|\psi\left(y_{t}, y_{u}\right)-\psi\left(y_{t}, \cdot\right)_{* y_{s}}\left[\psi\left(y_{s}, y_{u}\right)-\psi\left(y_{s}, y_{t}\right)\right]\right|_{g} \leq C_{\psi} \omega(s, u)^{3 / p}
$$

Proof. Around every point in $y([0, T])$, using our usual techniques, we can find a neighborhood $\mathcal{W}$ such that $\mathcal{W} \times \mathcal{W} \subseteq \mathcal{D}$ and maps to a convex open set by a chart. We can then use Remark 2.52 with a slightly modified version (which includes three variables instead of two) of Lemma 2.51 to create a global estimate. We can then choose a $\delta$ such that $u-s \leq \delta$ forces the path to lie within one of these sets $\mathcal{W}$. Therefore, it suffices to prove the estimate locally. However, we can push forward the metric and $\psi$ to a convex set on Euclidean space. The rest follows from the Lemma 3.28 and the fact that $\left|\psi\left(y_{s}, y_{t}\right)\right| \leq C \omega(s, t)^{1 / p}$ for all $|t-s| \leq \delta$ for some $C<\infty$ and $\delta>0$. 


\subsection{Proof of Theorem 3.26}

Proof of Theorem 3.26. Let $0 \leq s \leq t \leq u \leq T$. Throughout this proof, we will use the notation $\underset{i}{\approx}$ with respect to the times $s$ and $u$. To prove the statement, we need to show $\tilde{z}_{s, t}+\tilde{z}_{t, u} \approx \tilde{z}_{s, u}$. We begin by working on the three terms for $\tilde{z}_{t, u}$ in the following equation

$$
\tilde{z}_{t, u}=\alpha_{t}\left(\psi\left(y_{t}, y_{u}\right)\right)+\alpha_{t}^{\dagger}\left(I \otimes y_{t}^{\dagger}\right) \mathbb{X}_{t, u}+\alpha_{t}\left(S_{y_{t}}^{\mathcal{G}} \circ y_{t}^{\dagger \otimes 2} \mathbb{X}_{t, u}\right) .
$$

Using Corollary 3.29 followed by Corollary 3.16 we find

$$
\begin{aligned}
\alpha_{t}\left(\psi\left(y_{t}, y_{u}\right)\right) \underset{3}{\approx} & \alpha_{t} \psi\left(y_{t}, \cdot\right)_{* y_{s}}\left[\psi\left(y_{s}, y_{u}\right)-\psi\left(y_{s}, y_{t}\right)\right] \\
\underset{3}{\widetilde{\sigma}} & \alpha_{t} U\left(y_{t}, y_{s}\right)\left[I+S_{y_{s}}^{\mathcal{G}}\left(\psi\left(y_{s}, y_{t}\right) \otimes(\cdot)\right)\right]\left[\psi\left(y_{s}, y_{u}\right)-\psi\left(y_{s}, y_{t}\right)\right] \\
\underset{3}{\approx} & {\left[\alpha_{s}+\alpha_{s}^{\dagger} x_{s, t} \otimes(\cdot)\right]\left[I+S_{y_{s}}^{\mathcal{G}}\left(\psi\left(y_{s}, y_{t}\right) \otimes(\cdot)\right)\right]\left[\psi\left(y_{s}, y_{u}\right)-\psi\left(y_{s}, y_{t}\right)\right] } \\
\approx & \alpha_{s}\left[I+S_{y_{s}}^{\mathcal{G}}\left(\psi\left(y_{s}, y_{t}\right) \otimes(\cdot)\right)\right]\left[\psi\left(y_{s}, y_{u}\right)-\psi\left(y_{s}, y_{t}\right)\right] \\
& +\alpha_{s}^{\dagger} x_{s, t} \otimes\left[\psi\left(y_{s}, y_{u}\right)-\psi\left(y_{s}, y_{t}\right)\right] .
\end{aligned}
$$

Combining this equation with the estimates

$$
\psi\left(y_{s}, y_{t}\right) \underset{2}{\approx} y_{s}^{\dagger} x_{s, t} \text { and } \psi\left(y_{s}, y_{u}\right)-\psi\left(y_{s}, y_{t}\right) \underset{2}{\approx} y_{s}^{\dagger}\left[x_{s, u}-x_{s, t}\right]=y_{s}^{\dagger} x_{t, u}
$$

then shows,

$$
\alpha_{t}\left(\psi\left(y_{t}, y_{u}\right)\right) \underset{3}{\approx} \alpha_{s}\left[\psi\left(y_{s}, y_{u}\right)-\psi\left(y_{s}, y_{t}\right)\right]+\alpha_{s}\left(y_{s}^{\dagger}\right)^{\otimes 2} x_{s, t} \otimes x_{t, u}+\alpha_{s}^{\dagger}\left(I \otimes y_{s}^{\dagger}\right) x_{s, t} \otimes x_{t, u} .
$$

By the definitions of $C R P_{\mathbf{X}}(M)$ and $C R P_{y}^{U}(M, V)$ we have

$$
\begin{aligned}
\alpha_{t}^{\dagger}\left(I \otimes y_{t}^{\dagger}\right) \mathbb{X}_{t, u} & \underset{3}{\approx} \alpha_{t}^{\dagger}\left(I \otimes U\left(y_{t}, y_{s}\right) y_{s}^{\dagger}\right) \mathbb{X}_{t, u} \\
& =\alpha_{t}^{\dagger}\left(I \otimes U\left(y_{t}, y_{s}\right)\right)\left(I \otimes y_{s}^{\dagger}\right) \mathbb{X}_{t, u} \approx \widetilde{3}_{s}^{\dagger}\left(I \otimes y_{s}^{\dagger}\right) \mathbb{X}_{t, u}
\end{aligned}
$$

Lastly by the definitions of $C R P_{\mathbf{X}}(M)$ and $C R P_{y}^{U}(M, V)$ along with Lemma 3.27 with $\tilde{U}(m, n)=$ $\left(\psi_{m}\right)_{* n}$, we have and

$$
\begin{aligned}
\alpha_{t}\left(S_{y_{t}}^{\mathcal{G}} \circ y_{t}^{\dagger \otimes 2} \mathbb{X}_{t, u}\right) & \underset{3}{\approx} \alpha_{t}\left(S_{y_{t}}^{\mathcal{G}} \circ U\left(y_{t}, y_{s}\right)^{\otimes 2} \circ y_{s}^{\dagger \otimes 2} \mathbb{X}_{t, u}\right) \\
& \approx{ }_{3} \alpha_{t}\left(U\left(y_{t}, y_{s}\right) \circ S_{y_{s}}^{\mathcal{G}} \circ y_{s}^{\dagger \otimes 2} \mathbb{X}_{t, u}\right) \underset{3}{\approx} \alpha_{s}\left(S_{y_{s}}^{\mathcal{G}} \circ y_{s}^{\dagger \otimes 2} \mathbb{X}_{t, u}\right) .
\end{aligned}
$$

Adding together Eqs. 3.19 - 3.21 to

$$
\tilde{z}_{s, t}=\alpha_{s}\left(\psi\left(y_{s}, y_{t}\right)\right)+\alpha_{s}^{\dagger}\left(I \otimes y_{s}^{\dagger}\right) \mathbb{X}_{s, t}+\alpha_{s}\left(S_{y_{s}}^{\mathcal{G}} \circ y_{s}^{\dagger \otimes 2} \mathbb{X}_{s, t}\right)
$$

while making use Chen's identity in Eq. 2.2 shows

$$
\tilde{z}_{s, t}+\tilde{z}_{t, u} \underset{3}{\approx} \alpha_{s}\left(\psi\left(y_{s}, y_{u}\right)\right)+\alpha_{s}^{\dagger}\left(I \otimes y_{s}^{\dagger}\right) \mathbb{X}_{s, u}+\alpha_{s}\left(S_{y_{s}}^{\mathcal{G}} \circ y_{s}^{\dagger \otimes 2} \mathbb{X}_{s, u}\right)=\tilde{z}_{s, u}
$$




\subsection{A Map from $C R P_{y}^{U}(M, V)$ to $C R P_{y}^{\tilde{U}}(M, V)$}

Suppose that $\mathcal{G}=(\psi, U)$ and $\tilde{\mathcal{G}}=(\tilde{\psi}, \tilde{U})$ are two gauges on $M$. Generally, if $\boldsymbol{\alpha}:=\left(\alpha, \alpha^{\dagger}\right) \in$ $C R P_{y}^{U}(M, V)$, there is no reason to expect it also to be an element of $C R P_{y}^{\tilde{U}}(M, V)$. However, the main theorem [Theorem 3.32 of this section shows there is a "natural" bijection between $C R P_{y}^{U}(M, V)$ and $C R P_{y}^{\tilde{U}}(M, V)$ which preserves the notions of integration. The following proposition is needed in the proof of Theorem 3.32 and moreover motivates the statement of the theorem.

Proposition 3.30 If $\mathcal{G}=(\psi, U)$ and $\tilde{\mathcal{G}}=(\tilde{\psi}, \tilde{U})$ are two gauges on $M$ and $\mathbf{y}=\left(y, y^{\dagger}\right) \in$ $C R P_{\mathbf{X}}(M)$, then

$$
\mathbf{y}_{s, t}^{\mathcal{G}} \underset{3}{\approx} \mathbf{y}_{s, t}^{\tilde{\mathcal{G}}}+\left(S_{y_{s}}^{\tilde{U}, U}\left(\left(y_{s}^{\dagger}\right)^{\otimes 2} \mathbb{X}_{s, t}\right), 0\right)
$$

where $\mathbf{y}_{s, t}^{\mathcal{G}}$ and $\mathbf{y}_{s, t}^{\tilde{\mathcal{G}}}$ are as in Definition 3.20.

Proof. From Proposition 3.17

$$
\begin{aligned}
\psi\left(y_{s}, y_{t}\right)-\tilde{\psi}\left(y_{s}, y_{t}\right) & \underset{3}{\approx} \frac{1}{2} S_{y_{s}}^{\tilde{\psi}_{*}, \psi_{*}}\left(\psi\left(y_{s}, y_{t}\right) \otimes \psi\left(y_{s}, y_{t}\right)\right) \\
& \approx \frac{1}{3} \frac{1}{2} S_{y_{s}, \psi_{*}}\left(\left(y_{s}^{\dagger} \otimes y_{s}^{\dagger}\right)\left[x_{s, t} \otimes x_{s, t}\right]\right)=S_{y_{s}}^{\tilde{\psi}_{*}, \psi_{*}}\left(\left(y_{s}^{\dagger}\right)^{\otimes 2} \mathbb{X}_{s, t}\right)
\end{aligned}
$$

wherein we have used $S_{y_{s}}^{\tilde{\psi}_{*}, \psi_{*}}$ is symmetric and $\mathbf{X}=(x, \mathbb{X})$ is a weak-geometric rough path for the last equality. Making use of this estimate it now follows that

$$
\begin{aligned}
\mathbf{y}_{s, t}^{\mathcal{G}}-\mathbf{y}_{s, t}^{\tilde{\mathcal{G}}} & =\left(\psi\left(y_{s}, y_{t}\right)-\tilde{\psi}\left(y_{s}, y_{t}\right)+\left(S_{y_{s}}^{\mathcal{G}}-S_{y_{s}}^{\tilde{\mathcal{G}}}\right)\left(\left(y_{s}^{\dagger}\right)^{\otimes 2} \mathbb{X}_{s, t}\right), 0\right) \\
& \approx\left(\left(S_{y_{s}}^{\tilde{\psi}_{*}, \psi_{*}}+S_{y_{s}}^{\mathcal{G}}-S_{y_{s}}^{\tilde{\mathcal{G}}}\right)\left(\left(y_{s}^{\dagger}\right)^{\otimes 2} \mathbb{X}_{s, t}\right), 0\right) .
\end{aligned}
$$

On the other hand, by Lemma 3.11 .

$$
\begin{aligned}
& S^{\tilde{\psi}_{*}, \psi_{*}}=S^{\tilde{\psi}_{*}, \tilde{U}}+S^{\tilde{U}, \psi_{*}}=S^{\tilde{\psi}_{*}, \tilde{U}}+S^{\tilde{U}, U}+S^{U, \psi_{*}} \\
& =S^{\tilde{\mathcal{G}}}-S^{\mathcal{G}}+S^{\tilde{U}, U}
\end{aligned}
$$

which combined with Eq. 3.23 gives Eq. 3.22.

Corollary 3.31 The integral, $\int\left\langle\boldsymbol{\alpha}, d \mathbf{y}^{\mathcal{G}}\right\rangle$ only depends on the choice of parallelism $U$ and not on the logarithm used to make the gauge $\mathcal{G}=(\psi, U)$.

Proof. From Proposition 3.30 with $U=\tilde{U}$, it follows that

$$
\int_{s}^{t}\left\langle\boldsymbol{\alpha}, d \mathbf{y}^{\mathcal{G}}\right\rangle \underset{3}{\approx}\left\langle\boldsymbol{\alpha}_{s}, \mathbf{y}_{s, t}^{\mathcal{G}}\right\rangle \underset{3}{\approx}\left\langle\boldsymbol{\alpha}_{s}, \mathbf{y}_{s, t}^{\tilde{\mathcal{G}}}\right\rangle \underset{3}{\approx} \int_{s}^{t}\left\langle\boldsymbol{\alpha}, d \mathbf{y}^{\tilde{\mathcal{G}}}\right\rangle
$$

from which it follows that the two additive functionals, $\int\left\langle\boldsymbol{\alpha}, d \mathbf{y}^{\mathcal{G}}\right\rangle$ and $\int\left\langle\boldsymbol{\alpha}, d \mathbf{y}^{\tilde{\mathcal{G}}}\right\rangle$, must be equal.

If $\boldsymbol{\alpha}=\left(\alpha, \alpha^{\dagger}\right) \in C R P_{y}^{U}(M, V)$ and $U \neq \tilde{U}$, then

$$
\left\langle\boldsymbol{\alpha}_{s}, \mathbf{y}_{s, t}^{\mathcal{G}}\right\rangle \underset{3}{\approx}\left\langle\boldsymbol{\alpha}_{s}, \mathbf{y}_{s, t}^{\tilde{\mathcal{G}}}+\left(S_{y_{s}}^{\tilde{U}, U}\left(\left(y_{s}^{\dagger}\right)^{\otimes 2} \mathbb{X}_{s, t}\right), 0\right)\right\rangle=\left\langle\tilde{\boldsymbol{\alpha}}_{s}, \mathbf{y}_{s, t}^{\tilde{\mathcal{g}}}\right\rangle
$$

where $\tilde{\boldsymbol{\alpha}}_{s}$ is defined in Eq. 3.25 below. The identity in Eq. 3.24 suggests the following theorem. 
Theorem 3.32 The map

$$
\boldsymbol{\alpha}_{s}=\left(\alpha_{s}, \alpha_{s}^{\dagger}\right) \longrightarrow \tilde{\boldsymbol{\alpha}}_{s}:=\left(\tilde{\alpha}_{s}, \tilde{\alpha}_{s}^{\dagger}\right):=\left(\alpha_{s}, \alpha_{s}^{\dagger}+\alpha_{s} S_{y_{s}}^{\tilde{U}, U} y_{s}^{\dagger} \otimes I\right)
$$

is a bijection from $C R P_{y}^{U}(M, V)$ to $C R P_{y}^{\tilde{U}}(M, V)$ such that

$$
\int\left\langle\boldsymbol{\alpha}, d \mathbf{y}^{\mathcal{G}}\right\rangle=\int\left\langle\tilde{\boldsymbol{\alpha}}, d \mathbf{y}^{\tilde{\mathcal{G}}}\right\rangle
$$

Proof. The only thing that is really left to prove here is the assertion that $\tilde{\boldsymbol{\alpha}} \in C R P_{y}^{\tilde{U}}(M, V)$. First we prove that item 3 of Definition 3.1 holds for $\tilde{\boldsymbol{\alpha}}$.

From Theorem 3.15 with $m=y_{s}$ and $n=y_{t}$, we find

$$
U\left(y_{s}, y_{t}\right) \tilde{U}\left(y_{s}, y_{t}\right)^{-1} \underset{2}{\approx} I+S_{y_{s}}^{\tilde{U}, U}\left(\psi\left(y_{s}, y_{t}\right) \otimes(\cdot)\right)
$$

and then combining this result with Corollary 2.29 shows

$$
\tilde{U}\left(y_{t}, y_{s}\right) \underset{2}{\approx} U\left(y_{t}, y_{s}\right)\left[I+S_{y_{s}}^{\tilde{U}, U}\left(\psi\left(y_{s}, y_{t}\right) \otimes(\cdot)\right)\right] .
$$

From this equation and the fact that $\boldsymbol{\alpha} \in C R P_{y}^{U}(M, V)$, we learn

$$
\begin{aligned}
\alpha_{t} \tilde{U}\left(y_{t}, y_{s}\right)-\alpha_{s} & \underset{2}{\approx} \alpha_{t} U\left(y_{t}, y_{s}\right)\left[I+S_{y_{s}}^{\tilde{U}, U}\left(\psi\left(y_{s}, y_{t}\right) \otimes(\cdot)\right)\right]-\alpha_{s} \\
& \approx\left(\alpha_{s}+\alpha_{s}^{\dagger} x_{s, t}\right)\left[I+S_{y_{s}}^{\tilde{U}, U}\left(\psi\left(y_{s}, y_{t}\right) \otimes(\cdot)\right)\right]-\alpha_{s} \\
& \approx \alpha_{s}^{\dagger} x_{s, t}+\alpha_{s} S_{y_{s}}^{\tilde{U}, U}\left(y_{s}^{\dagger} x_{s, t} \otimes(\cdot)\right)=\tilde{\alpha}_{s}^{\dagger}\left(x_{s, t} \otimes(\cdot)\right)
\end{aligned}
$$

as desired.

Next we check item 4 of Definition 3.1. We are given

$$
\begin{aligned}
0 \approx & \approx \\
= & \alpha_{t}^{\dagger} \circ\left(I \otimes U\left(y_{t}, y_{s}\right)\right)-\alpha_{s}^{\dagger} \\
= & \tilde{\alpha}_{t}^{\dagger} \circ\left(I \otimes \tilde{U}\left(y_{t}, y_{s}\right)\right)-\tilde{\alpha}_{s}^{\dagger} \\
& -\alpha_{t} \circ S_{y_{t}}^{\tilde{U}, U} \circ\left(y_{t}^{\dagger} \otimes U\left(y_{t}, y_{s}\right)\right)+\alpha_{s} \circ S_{y_{s}}^{\tilde{U}, U} \circ\left(y_{s}^{\dagger} \otimes I\right)
\end{aligned}
$$

wherein we have used that $U\left(y_{s}, y_{t}\right) \approx \tilde{U}\left(y_{s}, y_{t}\right)$ (for example, see Eq. (3.27)). We therefore must show the last line is approximately 0 . However, by Lemma 3.27 we have $S_{y_{t}}^{\tilde{U}, U} \circ U\left(y_{t}, y_{s}\right)^{\otimes 2} \approx$ $U\left(y_{t}, y_{s}\right) \circ S_{y_{t}}^{\tilde{U}, U}$. Thus

$$
\begin{aligned}
& \alpha_{t} \circ S_{y_{t}}^{\tilde{U}, U} \circ\left(y_{t}^{\dagger} \otimes U\left(y_{t}, y_{s}\right)\right)-\alpha_{s} \circ S_{y_{s}}^{\tilde{U}, U} \circ\left(y_{s}^{\dagger} \otimes I\right) \\
& \quad \approx \alpha_{t} \circ S_{y_{t}}^{\tilde{U}, U} \circ\left(U\left(y_{t}, y_{s}\right) y_{s}^{\dagger} \otimes U\left(y_{t}, y_{s}\right)\right)-\alpha_{s} \circ S_{y_{s}}^{\tilde{U}, U} \circ\left(y_{s}^{\dagger} \otimes I\right) \\
& \quad \approx\left[\alpha_{t} \circ U\left(y_{t}, y_{s}\right)-\alpha_{s}\right]\left[S_{y_{s}}^{\tilde{U}, U} \circ\left(y_{s}^{\dagger} \otimes I\right)\right] \approx 0 .
\end{aligned}
$$




\section{Integrating One-Forms Along a CRP}

Lemma 4.1 Let $V$ be a Banach space and $U$ be a parallelism on $M$. If $\alpha \in \Omega^{1}(M, V)$ is a $V$ valued smooth one-form on $M$, then

$$
\alpha_{n} \circ U(n, m)-\alpha_{m}={ }_{2} \nabla_{\psi(m, n)}^{U} \alpha
$$

where $\nabla^{U}$ is the covariant derivative defined in Remark 2.20.

Proof. By definition, $\nabla_{v_{m}}^{U} \alpha$ is determined by the product rule,

$$
v_{m}[\alpha(Y)]=\left(\nabla_{v_{m}}^{U} \alpha\right)(Y(m))+\alpha_{m}\left(\nabla_{v_{m}}^{U} Y\right) .
$$

However, we may also write

$$
\begin{aligned}
v_{m}[\alpha(Y)] & =\left.\frac{d}{d t}\right|_{0} \alpha\left(U\left(m, \sigma_{t}\right)^{-1} U\left(m, \sigma_{t}\right) Y\left(\sigma_{t}\right)\right) \\
& =\left.\frac{d}{d t}\right|_{0} \alpha\left(U\left(m, \sigma_{t}\right)^{-1} Y(m)\right)+\alpha_{m}\left(\nabla_{v_{m}}^{U} Y\right)
\end{aligned}
$$

where $\sigma_{t}$ is such that $\dot{\sigma}_{0}=v_{m}$. Combining the last two facts shows that

$$
\nabla_{v_{m}}^{U} \alpha=\left.\frac{d}{d t}\right|_{0}\left[\alpha \circ U\left(m, \sigma_{t}\right)^{-1}\right]
$$

By Corollary 2.29, we may alternatively write Eq. 4.2 as

$$
\nabla_{v_{m}}^{U} \alpha=\left.\frac{d}{d t}\right|_{0}\left[\alpha \circ U\left(\sigma_{t}, m\right)\right] .
$$

To prove the lemma, we note this is a local result and we therefore may assume $M=\mathbb{R}^{d}$. Then by Taylor's theorem,

$$
\begin{aligned}
\alpha_{n} \circ U(n, m) & =\alpha_{m}+D\left[\alpha_{(\cdot)} \circ U(\cdot, m)\right](m)(n-m)+O\left(|n-m|^{2}\right) \\
& =\alpha_{m}+\nabla_{(n-m)_{m}}^{U} \alpha+O\left(|n-m|^{2}\right) \\
& =\alpha_{m}+\nabla_{\psi(m, n)}^{U} \alpha+O\left(|\psi(m, n)|^{2}\right) .
\end{aligned}
$$

Suppose that $\alpha \in \Omega^{1}(M, V)$ is a $V$ - valued one-form and $U$ is a parallelism on $M$. We wish to take $\alpha_{s}^{U}=\alpha_{y_{s}}:=\left.\alpha\right|_{T_{y_{s} M}}$. Making use of Lemma 4.1. we find

$$
\alpha_{t}^{U} \circ U\left(y_{t}, y_{s}\right)-\alpha_{s} \underset{2}{\approx} \nabla_{\psi\left(y_{s}, y_{t}\right)}^{U} \alpha \underset{2}{\approx} \nabla_{y_{s}^{\dagger} x_{s, t}}^{U} \alpha
$$

and this computation suggests the following proposition.

Proposition 4.2 Suppose that $\alpha \in \Omega^{1}(M, V)$ is a $V$ - valued one-form and $U$ is a parallelism on $M$, then

$$
\boldsymbol{\alpha}_{s}^{(y, U)}:=\left(\alpha_{y_{s}}, \alpha_{s}^{\dagger(y, U)}\right):=\left(\left.\alpha\right|_{T_{y_{s} M}}, \nabla_{y_{s}^{\dagger}(\cdot)}^{U} \alpha\right) \in C R P_{y}^{U}(M, V)
$$


Proof. In light of how $\boldsymbol{\alpha}_{s}^{y, U}$ has been defined and of Eq. 4.3, we need only verify Item 4 in Definition 3.1 is satisfied. To this end, suppose that $w \in W$, then

$$
\begin{aligned}
\alpha_{t}^{\dagger(y, U)} \circ\left(I \otimes U\left(y_{t}, y_{s}\right)\right)(w \otimes(\cdot)) & =\left(\nabla_{y_{t}^{\dagger} w}^{U} \alpha\right) U\left(y_{t}, y_{s}\right) \\
& \approx\left(\nabla_{U\left(y_{t}, y_{s}\right) y_{s}^{\dagger} w}^{U} \alpha\right) U\left(y_{t}, y_{s}\right)
\end{aligned}
$$

wherein we have used Inequality (2.23) along with Corollary 2.29 in the last line. Since for $v_{m} \in$ $T_{m} M$ the function $F(n):=\left(\nabla_{U(n, m) v_{m}}^{U} \alpha\right) U(n, m) \in L\left(T_{m} M, V\right)$ is smooth, it follows by Taylor's theorem that $F(n)={ }_{1} F(m)$ which translates to

$$
\left(\nabla_{U(n, m) v_{m}}^{U} \alpha\right) U(n, m)=_{1} \nabla_{v_{m}}^{U} \alpha .
$$

Taking $m=y_{s}, n=y_{t}$, and $v_{m}=y_{s}^{\dagger} w$ in this estimates shows

$$
\left(\nabla_{U\left(y_{t}, y_{s}\right) y_{s}^{\dagger} w}^{U} \alpha\right) U\left(y_{t}, y_{s}\right) \underset{1}{\approx} \nabla_{y_{s}^{\dagger} w}^{U} \alpha
$$

which combined with Eq. 4.4 completes the proof.

Theorem 4.3 If $\alpha \in \Omega^{1}(M, V)$ is a $V$ - valued one-form, then the integral $\int\left\langle\boldsymbol{\alpha}^{(y, U)}, d \mathbf{y}^{\mathcal{G}}\right\rangle$ is independent of any choice of gauge $\mathcal{G}=(\psi, U)$ on $M$. In the future we denote this integral more simply as $\int\langle\boldsymbol{\alpha}, d \mathbf{y}\rangle$.

Proof. Suppose that $U$ and $\tilde{U}$ are two parallelisms. According to Theorem 3.32 it suffices to show

$$
\alpha_{s}^{\dagger(y, \tilde{U})}=\alpha_{s}^{\dagger(y, U)}+\alpha_{y_{s}} S_{y_{s}}^{\tilde{U}, U}\left[y_{s}^{\dagger} \otimes I\right] .
$$

We will see that Eq. (4.5) is a fairly direct consequence of Example 3.10 which, when translated to the language of forms (see Eq. 4.1)), states

$$
\nabla_{v_{m}} \alpha=\tilde{\nabla}_{v_{m}} \alpha-\alpha \circ S_{m}^{\tilde{U}, U}\left(v_{m} \otimes(\cdot)\right) .
$$

So for $w \in W$, we have

$$
\begin{aligned}
\alpha_{s}^{\dagger(y, \tilde{U})} w & =\tilde{\nabla}_{y_{s}^{\dagger} w} \alpha=\nabla_{y_{s}^{\dagger} w} \alpha+\alpha_{y_{s}} S_{m}^{\tilde{U}, U}\left(y_{s}^{\dagger} w \otimes(\cdot)\right) \\
& =\alpha_{s}^{\dagger(y, U)} w+\alpha_{y_{s}} S_{m}^{\tilde{U}, U}\left(y_{s}^{\dagger} w \otimes(\cdot)\right)
\end{aligned}
$$

which proves Eq. 4.5.

Let us now record a number of possible different expressions for computing $\int_{s}^{t} \alpha(d \mathbf{y})$ depending on the choice of gauge we make.

Proposition 4.4 Let $\mathcal{G}=(\psi, U)$ be a gauge. There exists a $\delta>0$ such that for $s<t$ and $t-s<\delta$, the approximation

$$
\left[\int \alpha(d \mathbf{y})\right]_{s, t}^{1} \underset{3}{\approx} \alpha_{y_{s}}\left(\psi\left(y_{s}, y_{t}\right)\right)+\left[\left(\nabla_{(\cdot)}^{U} \alpha\right)_{y_{s}}+\alpha_{y_{s}} \circ S_{y_{s}}^{\mathcal{G}}\right] \circ y_{s}^{\dagger \otimes 2} \mathbb{X}_{s, t}
$$

holds. 
In the case that we take $U=U^{\psi}$, we get a slightly simpler formula.

Corollary 4.5 Let $\psi$ be a logarithm. There exists a $\delta>0$ such that for $s<t$ and $t-s<\delta$, the approximation

$$
\left[\int \alpha(d \mathbf{y})\right]_{s, t}^{1} \underset{3}{\widetilde{3}} \alpha_{y_{s}}\left(\psi\left(y_{s}, y_{t}\right)\right)+d\left(\alpha_{(\cdot)} \circ\left(\psi_{(\cdot)}\right)_{* y_{s}}\right)_{y_{s}} \circ y_{s}^{\dagger \otimes 2} \mathbb{X}_{s, t}
$$

holds.

Example 4.6 Let $\nabla$ be a covariant derivative on $M$. There exists a $\delta>0$ such that for $s<t$ and $t-s<\delta$, the approximation

$$
\left[\int \alpha(d \mathbf{y})\right]_{s, t}^{1} \underset{3}{\approx} \alpha_{y_{s}}\left(\left(\exp _{y_{s}}^{\nabla}\right)^{-1}\left(y_{t}\right)\right)+\left[(\nabla \alpha)_{y_{s}}+\frac{1}{2} \alpha_{y_{s}} \circ T_{y_{s}}^{\nabla}\right] \circ y_{s}^{\dagger \otimes 2} \mathbb{X}_{s, t}
$$

holds. Indeed this follows immediately from Proposition 4.4, Lemma 3.13, and the fact that

$$
\begin{aligned}
(\nabla \alpha)_{y_{s}}\left(v_{m}, w_{m}\right): & =v_{m}[\alpha(\boldsymbol{W})]-\alpha\left(\nabla_{v_{m}} \boldsymbol{W}\right) \\
& =d\left(\alpha_{(\cdot)} \circ \boldsymbol{W}(\cdot)\right)_{y_{s}}\left(v_{m}\right)-\alpha\left(\nabla_{v_{m}} \boldsymbol{W}\right)
\end{aligned}
$$

where $\boldsymbol{W}$ is any vector field such that $\boldsymbol{W}(m)=w_{m}$. Choosing $\boldsymbol{W}=U^{\nabla}(\cdot, m) w_{m}$, we have

$$
\nabla_{v_{m}} \boldsymbol{W}=\nabla_{v_{m}} U^{\nabla}(\cdot, m) w_{m}=0
$$

by the definition of parallel translation.

\subsection{Integration of a One-Form Using Charts}

It is easy to see that by independence of gauges, the integral of a one-form along $\left(y_{s}, y_{s}^{\dagger}\right)$ is an object which we only need to compute locally. As mentioned in Remark 2.22 we have an example of a local gauge by using a chart. Plugging this formula into the integral approximation from Corollary 4.5 , we get the following.

Corollary 4.7 Let $\phi$ be a chart on $M$. For all $a, b \in[0, T]$ such that $y[a, b] \subset D(\phi)$, we have the approximation

$$
\left[\int \alpha(d \mathbf{y})\right]_{s, t}^{1} \underset{3}{\approx} \alpha_{y_{s}}\left(\left(d \phi_{y_{s}}\right)^{-1}\left[\phi\left(y_{t}\right)-\phi\left(y_{s}\right)\right]\right)+d\left(\alpha_{(\cdot)} \circ\left(d \phi_{(\cdot)}\right)^{-1} d \phi_{y_{s}}\right)_{y_{s}} \circ y_{s}^{\dagger \otimes 2} \mathbb{X}_{s, t}
$$

holds for all $s<t \in[a, b]$.

Although this formula looks a bit complicated, it may be reduced to something that makes more sense. First, note that

$$
\alpha_{m} \circ\left(d \phi_{m}\right)^{-1}=\left[\left(\phi^{-1}\right)^{*} \alpha\right]_{\phi(m)} .
$$


Thus we can reduce the right hand side Eq. 4.77 to

$$
\begin{aligned}
& {\left[\left(\phi^{-1}\right)^{*} \alpha\right]_{\phi\left(y_{s}\right)}\left(\phi\left(y_{t}\right)-\phi\left(y_{s}\right)\right)+d\left(\left[\left(\phi^{-1}\right)^{*} \alpha\right]_{\phi(\cdot)} d \phi_{y_{s}}\right)_{y_{s}} \circ y_{s}^{\dagger \otimes 2} \mathbb{X}_{s, t}} \\
& =\left[\left(\phi^{-1}\right)^{*} \alpha\right]_{\phi\left(y_{s}\right)}\left(\phi\left(y_{t}\right)-\phi\left(y_{s}\right)\right)+\left[\left(\phi^{-1}\right)^{*} \alpha\right]_{\phi\left(y_{s}\right)}^{\prime}\left[d \phi_{y_{s}} \circ y_{s}^{\dagger}\right]^{\otimes 2} \mathbb{X}_{s, t} .
\end{aligned}
$$

Now, if we recall Notation 2.41, we see that this is approximately equal to another rough integral. More precisely

$$
\left[\int \alpha(d \mathbf{y})\right]_{s, t}^{1} \underset{3}{\approx}\left[\int\left(\phi^{-1}\right)^{*} \alpha\left(d \phi_{*} \mathbf{y}\right)\right]_{s, t}^{1} .
$$

However, additive functionals are unique up to this order, so in fact

$$
\left[\int \alpha(d \mathbf{y})\right]_{s, t}^{1}=\left[\int\left(\phi^{-1}\right)^{*} \alpha\left(d \phi_{*} \mathbf{y}\right)\right]_{s, t}^{1}
$$

which is a relation which should hold under any reasonable integral. This is summarized in the following theorem which gives us an alternative way of defining this integral.

Theorem 4.8 The integral, $\int \alpha(d \mathbf{y})$, is the unique $V$ - valued rough path controlled by $\mathbf{X}$ on $[0, T]$ starting at 0 determined by

1. $\left[\int \alpha(d \mathbf{y})\right]_{s, t}^{1}=\left[\int\left(\left(\phi^{-1}\right)^{*} \alpha\right)\left(d \phi_{*} \mathbf{y}\right)\right]_{s, t}^{1}$ for any chart and $s<t \in[0, T]$ such that $y([s, t]) \subset$ $D(\phi)$

2. $\left[\int \alpha(d \mathbf{y})\right]_{s}^{\dagger}=\alpha_{y_{s}} \circ y_{s}^{\dagger}$.

[See Theorem 4.15 below for a more general version of this theorem.]

The next theorem with our current toolset can now be proved in two different ways. We can reduce the result to a special case of Theorem 3.24 or, by using the chart definitions of integration along a one-form, can reduce it to its validity in the flat case. The first method is quick but may hide the concept of what is happening. We therefore provide both proofs.

Theorem 4.9 (Associativity Theorem II) Suppose that $y \in C R P(M), \alpha \in \Omega^{1}(M, V)$, and $K: M \rightarrow L(V, \tilde{V})$ is a smooth function so that $K \alpha \in \Omega^{1}(M, \tilde{V})$. If $\mathbf{z}=\int \alpha(d \mathbf{y}) \in C R P(V)$, then

$$
\int(K \alpha)(d \mathbf{y})=\int\left\langle K_{*}(\mathbf{y}), d \mathbf{z}\right\rangle \quad\left(=: \int\left\langle K_{*}(\mathbf{y}), d \int \alpha(d \mathbf{y})\right\rangle\right),
$$

where $K_{*}(\mathbf{y})=\left(K(y), K_{* y} y^{\dagger}\right) \in C R P_{\mathbf{X}}\left(\operatorname{Hom}\left(V, V^{\prime}\right)\right)$.

Proof. Method 1: Letting $\mathcal{G}=(\psi, U)$ be any gauge, we define $\mathbf{f}:=\left(f, f^{\dagger}\right) \in C R P_{\mathbf{X}}(\operatorname{Hom}(V, \tilde{V}))$ by the formula

$$
f_{s}:=K\left(y_{s}\right) \quad \text { and } \quad f_{s}^{\dagger}:=K_{* y_{s}} y_{s}^{\dagger}
$$


and $\boldsymbol{\alpha}^{(y, U)}$ as in Proposition 4.2 (see Proposition 4.10 below to see why $\mathbf{f} \in C R P_{\mathbf{X}}(\operatorname{Hom}(V, \tilde{V}))$ ). Then by Theorem 3.24 , we have

$$
\int\left\langle\mathbf{f} \boldsymbol{\alpha}^{(y, U)}, d \mathbf{y}^{\mathcal{G}}\right\rangle=\int\langle\mathbf{f}, d \mathbf{z}\rangle
$$

where $\mathbf{z}=\int\left\langle\boldsymbol{\alpha}^{(y, U)}, d \mathbf{y}^{\mathcal{G}}\right\rangle=\int \alpha(d \mathbf{y})$. The right hand side in Equation 4.8 is simply $\int\left\langle K_{*}(\mathbf{y}), d \mathbf{z}\right\rangle$ while the $\mathbf{f} \boldsymbol{\alpha}^{(y, U)}$ term on the left hand side can be recognized as $(K \alpha)^{(y, U)}$. Indeed, by the product rule with $\nabla^{U}$, we have

$$
\begin{aligned}
(K \alpha)_{s}^{(y, U)} & =\left(\left.K\left(y_{s}\right) \alpha\right|_{T_{y_{s}} M}, \nabla_{y_{s}^{\dagger}(\cdot)}^{U}[K(\cdot) \alpha]\right) \\
& =\left(\left.K \alpha\right|_{T_{y_{s}} M}, K_{* y_{s}} y_{s}^{\dagger} \alpha+K\left(y_{s}\right) \nabla_{y_{s}^{\dagger}(\cdot)}^{U} \alpha\right) \\
& =\left(f_{s} \alpha_{s}, f_{s}^{\dagger} \alpha+f_{s} \alpha_{s}^{\dagger(y, U)}\right) \\
& =\mathbf{f} \boldsymbol{\alpha}^{(y, U)} .
\end{aligned}
$$

Thus

$$
\int(K \alpha)(d \mathbf{y}):=\int\left\langle(K \alpha)_{s}^{(y, U)}, d \mathbf{y}^{\mathcal{G}}\right\rangle=\int\left\langle\mathbf{f} \boldsymbol{\alpha}^{(y, U)}, d \mathbf{y}^{\mathcal{G}}\right\rangle=\int\left\langle K_{*}(\mathbf{y}), d \mathbf{z}\right\rangle .
$$

Method 2: By a simple patching argument, this is really a local result and hence using the chart definitions of integration it suffices to check this result in the case $M$ is an open subset of $\mathbb{R}^{d}$. First we check the derivative processes. From the definitions we have

$$
z_{s}^{\dagger}=\alpha_{y_{s}} \circ y_{s}^{\dagger} \quad \text { and } \quad\left[\int(K \alpha)(d \mathbf{y})\right]_{s}^{\dagger}=(K \alpha)_{y_{s}} \circ y_{s}^{\dagger}=K\left(y_{s}\right) \alpha_{y_{s}} \circ y_{s}^{\dagger}=K\left(y_{s}\right) z_{s}^{\dagger} .
$$

Thus

$$
\left[\int(K \alpha)(d \mathbf{y})\right]_{s}^{\dagger}=K\left(y_{s}\right) z_{s}^{\dagger}
$$

On the other hand

$$
\left[\int\left\langle K_{*}(\mathbf{y}), d \mathbf{z}\right\rangle\right]_{s}^{\dagger}=[K(y)]_{s} z_{s}^{\dagger}=K\left(y_{s}\right) z_{s}^{\dagger}
$$

Similarly for the paths

$$
z_{s, t} \underset{3}{\approx} \alpha\left(y_{s, t}\right)+\alpha_{y_{s}}^{\prime} y_{s}^{\dagger \otimes 2} \mathbb{X}_{s, t} .
$$

and so

$$
\begin{aligned}
{\left[\int(K \alpha)(d \mathbf{y})\right]_{s, t}^{1} } & \underset{3}{3}(K \alpha)_{y_{s}} y_{s, t}+(K \alpha)_{y_{s}}^{\prime} y_{s}^{\dagger \otimes 2} \mathbb{X}_{s, t} \\
& =K\left(y_{s}\right) \alpha_{y_{s}} y_{s, t}+K\left(y_{s}\right) \alpha_{y_{s}}^{\prime} y_{s}^{\dagger \otimes 2} \mathbb{X}_{s, t}+\left[K_{y_{s}}^{\prime}\left(y_{s}^{\dagger}(\cdot) \otimes \alpha y_{s}^{\dagger}(\cdot)\right)\right] \mathbb{X}_{s, t} \\
& \approx K\left(y_{s}\right) z_{s, t}+K_{y_{s}}^{\prime}\left(y_{s}^{\dagger} \otimes z_{s}^{\dagger}\right) \mathbb{X}_{s, t}
\end{aligned}
$$


On the other hand

$$
\begin{aligned}
{\left[\int\left\langle K_{*}(\mathbf{y}), d \mathbf{z}\right\rangle\right]_{s, t}^{1} } & \underset{3}{\approx} K\left(y_{s}\right) z_{s, t}+\left[K_{*}(\mathbf{y})\right]_{s}^{\dagger} z_{s}^{\dagger} \mathbb{X}_{s, t} \\
& =K\left(y_{s}\right) z_{s, t}+K_{y_{s}}^{\prime}\left(y_{s}^{\dagger} \otimes z_{s}^{\dagger}\right) \mathbb{X}_{s, t} .
\end{aligned}
$$

Comparing these expressions completes the proof.

\subsection{Push-forwards of Controlled Rough Paths}

Let $M=M^{d}$ and $\tilde{M}=\tilde{M}^{\tilde{d}^{\prime}}$ be manifolds. Let $f: M \rightarrow \tilde{M}$ be smooth and suppose $\mathbf{y}_{s}=\left(y_{s}, y_{s}^{\dagger}\right)$ $\in C R P_{\mathbf{X}}(M)$. In Definition 4.11 below, we are going to give a definition of the push-forward of $\mathbf{y}$ by $f$ which generalizes Example 2.56

Proposition 4.10 The pair $\left(f\left(y_{s}\right), f_{*} \circ y_{s}^{\dagger}\right)$ is an element of $C R P_{\mathbf{X}}(\tilde{M})$.

Proof. Suppose $\tilde{\phi}$ is a chart on $\tilde{M}$ such that $f \circ y([a, b]) \subseteq D(\tilde{\phi})$. We must show that

$$
\left|\tilde{\phi} \circ f\left(y_{t}\right)-\tilde{\phi} \circ f\left(y_{s}\right)-d \tilde{\phi} \circ f_{*} y_{s}^{\dagger} x_{s, t}\right| \leq C_{\tilde{\phi}, a, b} \omega(s, t)^{2 / p}
$$

and

$$
\left|d \tilde{\phi} \circ f_{*} y_{t}^{\dagger}-d \tilde{\phi} \circ f_{*} y_{s}^{\dagger}\right| \leq C_{\tilde{\phi}, a, b} \omega(s, t)^{1 / p}
$$

hold for some $C_{\tilde{\phi}, a, b}$ for all $s \leq t$ in $[a, b]$. We can again use our proof strategy outlined in Remark 2.52 to treat this problem in nice neighborhoods. We leave it to the reader to follow the pattern of earlier proofs to see that we can assume without loss of generality that there is a chart $\phi$ on $M$ such that $y([a, b]) \subseteq D(\phi)$ and $R(\phi)$ is convex. Which these simplifications, we note that $\left(z_{s}, z_{s}^{\dagger}\right):=\left(\phi\left(y_{s}\right), d \phi \circ y_{s}^{\dagger}\right)$ is a controlled rough path on $R(\phi)$ and the function $F:=\tilde{\phi} \circ f \circ \phi^{-1}: R(\phi) \rightarrow R(\tilde{\phi})$ is a map between Euclidean spaces. Therefore Inequalities 4.9 and 4.10 reduce to the fact that the pair $\left(F\left(z_{s}\right), F^{\prime}\left(z_{s}\right) \circ z_{s}^{\dagger}\right)$ is a controlled rough path in $\mathbb{R}^{d}$ (which is trivial by applying Taylor's theorem after we check that we get the correct terms); indeed, by a simple computation, we have

$$
\begin{aligned}
F^{\prime}\left(z_{s}\right) \circ z_{s}^{\dagger} & =d \tilde{\phi} \circ f_{*} \circ\left(d \phi^{-1}\right)_{z_{s}} \circ d \phi_{y_{s}} \circ y_{s}^{\dagger} \\
& =d \tilde{\phi} \circ f_{*} \circ\left(d \phi_{y_{s}}\right)^{-1} \circ d \phi_{y_{s}} \circ y_{s}^{\dagger} \\
& =d \tilde{\phi} \circ f_{*} y_{s}^{\dagger}
\end{aligned}
$$

and clearly $F\left(z_{s}\right)=\tilde{\phi} \circ f\left(y_{s}\right)$.

Definition 4.11 The push-forward of $\mathbf{y}$ denoted by $f_{*} \mathbf{y}$ or $f_{*}\left(y, y^{\dagger}\right)$ is the rough path controlled by $\mathbf{X}$ with path $f\left(y_{s}\right)$ and derivative process $f_{*} \circ y_{s}^{\dagger}$. If $\tilde{M}=\mathbb{R}^{\tilde{d}}$, we will abuse notation an write $f_{*} \mathbf{y}_{s}$ to mean $\left(f\left(y_{s}\right), d f \circ y_{s}^{\dagger}\right)$ (i.e. we forget the base point on the derivative process).

Remark 4.12 The push-forward operation on elements in $C R P_{\mathbf{X}}(M)$ is clearly covariant, i.e. if $f: M \rightarrow N$ and $g: N \rightarrow P$ are two smooth maps of manifolds, $M, N$, and $P$, then $(g \circ f)_{*}(\mathbf{y})=$ $g_{*}\left(f_{*}(\mathbf{y})\right)$. 
This definition is consistent with how we defined the integral of a one-form along a controlled rough path in the sense that we have a fundamental theorem of calculus. Let $V$ be a Banach space.

Theorem 4.13 Let $\mathbf{y}_{s}=\left(y_{s}, y_{s}^{\dagger}\right) \in C R P_{\mathbf{X}}(M)$ and $f$ be a smooth function from $M$ to $V$. Then

$$
f\left(y_{s}\right)-f\left(y_{0}\right)=\left[\int d f[d \mathbf{y}]\right]_{0, s}^{1}
$$

where $d f$ is interpreted as a one-form. Since we have $d f \circ y_{s}^{\dagger}=\left[\int d f[d \mathbf{y}]\right]_{s}^{\dagger}$ we have the equality

$$
f_{*}\left(y, y^{\dagger}\right)-\left(f\left(y_{0}\right), 0\right)=\int d f(d \mathbf{y})
$$

Proof. Although there are ways to do this proof without much machinery, we find it more instructive to work on a Riemannian manifold with the Levi-Civita covariant derivative. Since we have proved that the integral is independent of choice of metric, it does not matter which one we pick. With this in mind, we have the approximation

$$
\left[\int d f[d \mathbf{y}]\right]_{s, t}^{1} \underset{3}{\widetilde{3}} d f_{y_{s}}\left(\exp _{y_{s}}^{-1}\left(y_{t}\right)\right)+(\nabla d f)_{y_{s}}\left[y_{s}^{\dagger \otimes 2} \mathbb{X}_{s, t}\right]
$$

and as $\nabla d f$ is symmetric, it follows that

$$
\begin{aligned}
& {\left[\int d f[d \mathbf{y}]\right]_{s, t}^{1} \underset{3}{\widetilde{3}} d f_{y_{s}}\left(\exp _{y_{s}}^{-1}\left(y_{t}\right)\right)+\frac{1}{2}(\nabla d f)_{y_{s}}\left[y_{s}^{\dagger \otimes 2}\left(x_{s, t} \otimes x_{s, t}\right)\right]} \\
& \underset{3}{\approx} d f_{y_{s}}\left(\exp _{y_{s}}^{-1}\left(y_{t}\right)\right)+\frac{1}{2}(\nabla d f)_{y_{s}}\left[\exp _{y_{s}}^{-1}\left(y_{t}\right)^{\otimes 2}\right] \\
& \underset{3}{\approx} f\left(y_{t}\right)-f\left(y_{s}\right) \text {. }
\end{aligned}
$$

The last approximation above follows from Taylor's Theorem on manifolds (Theorem 6.1 in the Appendix). Note here that $f\left(y_{t}\right)-f\left(y_{s}\right)$ is additive so that

$$
\left[\int d f[d \mathbf{y}]\right]_{s, t}^{1}=f\left(y_{t}\right)-f\left(y_{s}\right) .
$$

Remark 4.14 If $M \subseteq$ is an embedded submanifold of $W=\mathbb{R}^{k},\left(y_{s}, y_{s}^{\dagger}\right) \in C R P_{\mathbf{X}}(M), I: M \rightarrow W$ denotes the identity (or embedding) map, and $\left(z_{s}, z_{s}^{\dagger}\right):=I_{*}\left(y_{s}, y_{s}^{\dagger}\right)$, then we have

$$
z_{s}=y_{s} \quad \text { and } \quad z_{s}^{\dagger}=\pi_{2} \circ y_{s}^{\dagger}
$$

where $\pi_{2}$ is the projection of the tangent vector component (i.e. it forgets the base point). We can associate to it a unique rough path $(y, \mathbb{Y})$ in $W$ such that

$$
\left(z_{s}^{\dagger} \otimes z_{s}^{\dagger}\right) \mathbb{X}_{s, t} \underset{3}{\approx} \mathbb{Y}_{s, t} .
$$

In this case, this is a rough path in the embedded sense (See [3]) since

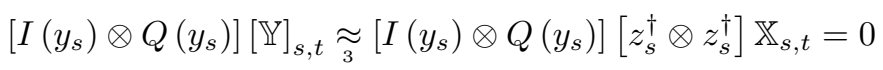

as $Q\left(y_{s}\right) \circ z_{s}^{\dagger}=0$ where $Q=I-P$ and $P(x)$ is orthogonal projection onto the tangent space at $x$. 
Lastly, we have a relation between push-forwards of paths and pull-backs of one-forms.

Theorem 4.15 (Push me-Pull me) Let $f: M \rightarrow \tilde{M}$, let $\mathbf{y}_{s}=\left(y_{s}, y_{s}^{\dagger}\right) \in C R P_{\mathbf{X}}(M)$ and let $\tilde{\alpha} \in \Omega^{1}(\tilde{M}, V)$. Then

Moreover

$$
\left[\int f^{*} \alpha(d \mathbf{y})\right]^{1}=\left[\int \alpha\left(d\left(f_{*} \mathbf{y}\right)\right)\right]^{1}
$$

$$
\int f^{*} \alpha(d \mathbf{y})=\int \alpha\left(d\left(f_{*} \mathbf{y}\right)\right)
$$

Proof. This is a statement we only have to prove locally. Indeed for each $s \in[0, T]$, there are charts $\phi^{s}$ and $\tilde{\phi}^{s}$ on $M$ and $\tilde{M}$ respectively such that $y_{s} \in D\left(\phi^{s}\right)$ and $f\left(y_{s}\right) \in D\left(\tilde{\phi}^{s}\right)$ which are open. We take $\mathcal{U}_{s}:=f^{-1}\left(D\left(\tilde{\phi}^{s}\right)\right) \cap D\left(\phi^{s}\right)$ and shrink it if necessary so that $\mathcal{V}_{s}=\phi\left(\mathcal{U}_{s}\right)$ is convex. Thus if we can prove that Eq. 4.11) holds whenever $y([a, b]) \subseteq \mathcal{U}$ such that $\phi(\mathcal{U})$ is convex and such that $f(y([a, b])) \subseteq D(\tilde{\phi})$, we will be done. We do this now:

By Theorem 4.8, the fact that pull-backs are contravariant, and that push-forwards are covariant, we have

$$
\begin{aligned}
{\left[\int f^{*} \alpha(d \mathbf{y})\right]_{s, t}^{1} } & =\left[\int\left(\phi^{-1}\right)^{*} f^{*} \alpha\left(d \phi_{*} \mathbf{y}\right)\right]_{s, t}^{1} \\
& =\left[\int\left(f \circ \phi^{-1}\right)^{*} \alpha\left(d \phi_{*} \mathbf{y}\right)\right]_{s, t}^{1} \\
& =\left[\int\left(\tilde{\phi}^{-1} \circ \tilde{\phi} \circ f \circ \phi^{-1}\right)^{*} \alpha\left(d \phi_{*} \mathbf{y}\right)\right]_{s, t}^{1} \\
& =\left[\int\left(\tilde{\phi} \circ f \circ \phi^{-1}\right)^{*}\left(\left(\tilde{\phi}^{-1}\right)^{*} \alpha\right)\left(d \phi_{*} \mathbf{y}\right)\right]_{s, t}^{1} \\
& =\left[\int\left(\tilde{\phi}^{-1}\right)^{*} \alpha\left(d\left(\left(\tilde{\phi} \circ f \circ \phi^{-1}\right)_{*} \phi_{*} \mathbf{y}\right)\right)\right]_{s, t}^{1}
\end{aligned}
$$

where the last step is just Eq. (4.11) on Euclidean space. This is a simple computation (for example, see the appendix of $[3]$ ). Thus, we have

$$
\begin{aligned}
{\left[\int f^{*} \alpha(d \mathbf{y})\right]_{s, t}^{1} } & =\left[\int\left(\tilde{\phi}^{-1}\right)^{*} \alpha\left(d\left(\left(\tilde{\phi} \circ f \circ \phi^{-1}\right)_{*} \phi_{*} \mathbf{y}\right)\right)\right]_{s, t}^{1} \\
& =\left[\int\left(\tilde{\phi}^{-1}\right)^{*} \alpha\left(d\left(\tilde{\phi}_{*}\left(f_{*} \mathbf{y}\right)\right)\right)\right]_{s, t}^{1} \\
& =\left[\int \alpha\left(d\left(f_{*} \mathbf{y}\right)\right)\right]_{s, t}^{1} .
\end{aligned}
$$

The fact that

$$
\left[\int f^{*} \alpha(d \mathbf{y})\right]^{\dagger}=\left[\int \alpha\left(d\left(f_{*} \mathbf{y}\right)\right)\right]^{\dagger}
$$

is trivial. 


\section{Rough Differential Equations}

Before discussing rough differential equations on a manifold, we will give an equivalent condition for a controlled rough path $\mathbf{z} \in C R P_{\mathbf{X}}\left(\mathbb{R}^{d}\right)$ to satisfy the RDE approximation on a compact interval in the flat case using logarithms.

For the next proposition, let $\psi$ be a logarithm on $\mathbb{R}^{d}$ such that $\psi(x, y)=(x, \bar{\psi}(x, y))$.

Proposition 5.1 Let $z:[a, b] \rightarrow \mathbb{R}^{d}$ be a path and let $\mathcal{W} \subseteq \mathbb{R}^{d}$ be an open convex set such that $z([a, b]) \subseteq \mathcal{W}$ and $\mathcal{W} \times \mathcal{W} \subseteq D(\psi)$. Then

$$
z_{s, t} \underset{3}{\approx} F_{x_{s, t}}\left(z_{s}\right)+\left.\left(\partial_{F_{w}\left(z_{s}\right)} F_{\tilde{w}}\right)\left(z_{s}\right)\right|_{w \otimes \tilde{w}=\mathbb{X}_{s, t}}
$$

if and only if

$$
\bar{\psi}\left(z_{s}, z_{t}\right) \underset{3}{\approx} F_{x_{s, t}}\left(z_{s}\right)+\left.\left(\partial_{F_{w}\left(z_{s}\right)}\left[\bar{\psi}_{z_{s}}^{\prime}(\cdot) F_{\tilde{w}}(\cdot)\right]\right)\left(z_{s}\right)\right|_{w \otimes \tilde{w}=\mathbb{X}_{s, t}}
$$

Proof. If $z$. satisfies Eq. (5.1), then from Eq. 2.17) of Theorem 2.24 with $y=z_{t}$ and $x=z_{s}$ we find,

$$
\begin{aligned}
\bar{\psi}\left(z_{s,} z_{t}\right) & =z_{s, t}+\frac{1}{2} \bar{\psi}_{x}^{\prime \prime}(x)\left(z_{s, t}\right)^{\otimes 2}+C\left(z_{s}, z_{t}\right)\left(z_{s, t}\right)^{\otimes 3} \\
& \approx F_{x_{s, t}}\left(z_{s}\right)+\left.\left(\partial_{F_{w}\left(z_{s}\right)} F_{\tilde{w}}\right)\left(z_{s}\right)\right|_{w \otimes \tilde{w}=\mathbb{X}_{s, t}}+\frac{1}{2} \bar{\psi}_{z_{s}}^{\prime \prime}\left(z_{s}\right)\left[F_{x_{s, t}}\left(z_{s}\right)\right]^{\otimes 2},
\end{aligned}
$$

wherein $C$ is a smooth function and we have made use of the fact that $z_{s, t} \approx 0$. By the product rule and the fact that $\psi$ is a logarithm it follows that

$$
\begin{aligned}
\left(\partial_{F_{w}\left(z_{s}\right)}\left[\bar{\psi}_{z_{s}}^{\prime}(\cdot) F_{\tilde{w}}(\cdot)\right]\right)\left(z_{s}\right) & =\bar{\psi}_{z_{s}}^{\prime \prime}\left(z_{s}\right) F_{w}\left(z_{s}\right) \otimes F_{\tilde{w}}\left(z_{s}\right)+\bar{\psi}_{z_{s}}^{\prime}\left(z_{s}\right)\left(\partial_{F_{w}\left(z_{s}\right)} F_{\tilde{w}}\right)\left(z_{s}\right) \\
& =\bar{\psi}_{z_{s}}^{\prime \prime}\left(z_{s}\right) F_{w}\left(z_{s}\right) \otimes F_{\tilde{w}}\left(z_{s}\right)+\left(\partial_{F_{w}\left(z_{s}\right)} F_{\tilde{w}}\right)\left(z_{s}\right) .
\end{aligned}
$$

Since $\mathbf{X}$ is a weak-geometric rough path and $\bar{\psi}_{z_{s}}^{\prime \prime}\left(z_{s}\right)$ is symmetric, we also have,

$$
\left.\bar{\psi}_{z_{s}}^{\prime \prime}\left(z_{s}\right) F_{w}\left(z_{s}\right) \otimes F_{\tilde{w}}\left(z_{s}\right)\right|_{\left.\right|_{w \otimes \tilde{w}=\mathbb{X}_{s, t}}}=\frac{1}{2} \bar{\psi}_{z_{s}}^{\prime \prime}\left(z_{s}\right)\left[F_{x_{s, t}}\left(z_{s}\right)\right]^{\otimes 2}
$$

which combined with Eq. 5.5 shows,

$$
\begin{aligned}
& \left.\left(\partial_{F_{w}\left(z_{s}\right)}\left[\bar{\psi}_{z_{s}}^{\prime}(\cdot) F_{\tilde{w}}(\cdot)\right]\right)\left(z_{s}\right)\right|_{w \otimes \tilde{w}=\mathbb{X}_{s, t}} \\
& \quad=\left.\left(\partial_{F_{w}\left(z_{s}\right)} F_{\tilde{w}}\right)\left(z_{s}\right)\right|_{w \otimes \tilde{w}=\mathbb{X}_{s, t}}+\frac{1}{2} \bar{\psi}_{z_{s}}^{\prime \prime}\left(z_{s}\right)\left[F_{x_{s, t}}\left(z_{s}\right)\right]^{\otimes 2} .
\end{aligned}
$$

Equation 5.2 now follows directly from Eqs. (5.4) and (5.6).

Conversely, now assume that Eq. 5.2 holds. From Eq. 5.2 and the fact that $\mathbf{X}$ is a rough path there exists $C_{1}<\infty$ such that $\left|\bar{\psi}\left(z_{s}, z_{t}\right)\right| \leq C_{1} \omega(s, t)^{1 / p}$. Combining this observation with Eq. 5.3 easily implies $z_{s, t} \approx 0$. Indeed, by uniform continuity, there exists a $\delta>0$ such that if $|t-s| \leq \delta$, we have

$$
\begin{aligned}
\left|z_{s, t}\right| & \leq\left|\bar{\psi}\left(z_{s}, z_{t}\right)\right|+\left|\frac{1}{2} \psi_{z_{s}}^{\prime \prime}\left(z_{s}\right)\left(z_{s, t}\right)^{\otimes 2}+C\left(z_{s}, z_{t}\right)\left(z_{s, t}\right)^{\otimes 3}\right| \\
& \leq C_{1} \omega(s, t)^{1 / p}+\frac{1}{2}\left|z_{s, t}\right|
\end{aligned}
$$


By using an argument similar to the proof of Theorem 2.47 we can bootstrap these local inequalities to prove the existence of a $C_{2}<\infty$ such that $\left|z_{s, t}\right| \leq C_{2} \omega(s, t)^{1 / p}$ for $a \leq s \leq t \leq b$.

From Eqs. 5.3 and 5.2 ,

$$
\begin{aligned}
z_{s, t} & =\bar{\psi}\left(z_{s}, z_{t}\right)-\frac{1}{2} \bar{\psi}_{z_{s}}^{\prime \prime}\left(z_{s}\right)\left(\psi\left(z_{s}, z_{t}\right)\right)^{\otimes 2}+C\left(z_{s}, z_{t}\right)\left(z_{s, t}\right)^{\otimes 3} \\
& \approx F_{x_{s, t}}\left(z_{s}\right)+\left.\left(\partial_{F_{w}\left(z_{s}\right)}\left[\bar{\psi}_{z_{s}}^{\prime}(\cdot) F_{\tilde{w}}(\cdot)\right]\right)\left(z_{s}\right)\right|_{w \otimes \tilde{w}=\mathbb{X}_{s, t}}-\frac{1}{2} \bar{\psi}_{z_{s}}^{\prime \prime}\left(z_{s}\right)\left(F_{x_{s, t}}\left(z_{s}\right)\right)^{\otimes 2} \\
& =F_{x_{s, t}}\left(z_{s}\right)+\left(\partial_{F_{w}\left(z_{s}\right)} F_{\tilde{w}}\right)\left(z_{s}\right)
\end{aligned}
$$

wherein we have used Eq. 5.6 for the last equality.

\subsection{RDEs on a Manifold}

We now move to the manifold case. Let $F: M \rightarrow L(W, T M)$ be smooth such that $F(m) \in$ $L\left(W, T_{m} M\right)$. Alternatively we can think of $F: W \rightarrow \Gamma(T M)$ where the map $w \rightarrow F_{w}(\cdot)$ is linear. We wish to give meaning to the differential equation

$$
d \mathbf{y}_{t}=F_{d \mathbf{x}_{t}}\left(y_{t}\right)
$$

with initial condition $y_{0}=\bar{y}_{0}$. To do this, first recall that any vector field can be transferred to Euclidean space by using charts. If $\mathcal{U} \subseteq D(\phi)$ where $\phi$ is a chart and $\mathcal{V}:=\phi(\mathcal{U})$ then

$$
F^{\phi}:=d \phi \circ\left(F \circ \phi^{-1}\right)
$$

is a vector field on $\mathcal{V}$ (which does not carry the base point). If $\mathbf{y}_{t}$ is to "solve" (5.8) then $\mathbf{z}_{t}:=\phi_{*} \mathbf{y}_{t}$ should solve the differential equation

$$
d \mathbf{z}_{t}=F_{d \mathbf{X}_{t}}^{\phi}\left(z_{t}\right)
$$

In the Euclidean case, Equation 5.9$]$ is satisfied if

$$
\begin{aligned}
& z_{t} \underset{3}{\widetilde{3}} z_{s}+F_{x_{s, t}}^{\phi}\left(z_{s}\right)+\left.\left(\partial_{F_{w}^{\phi}\left(z_{s}\right)} F_{\tilde{w}}^{\phi}\right)\left(z_{s}\right)\right|_{w \otimes \tilde{w}=\mathbb{X}_{s, t}} \\
& z_{s}^{\dagger}=F_{(\cdot)}^{\phi}\left(z_{s}\right)
\end{aligned}
$$

By writing out Equation 5.10 we have

$$
\begin{aligned}
\phi\left(y_{t}\right) \underset{3}{\approx} \phi\left(y_{s}\right)+d \phi \circ F_{x_{s, t}}\left(y_{s}\right)+\left.\left(\partial_{d \phi \circ F_{w}\left(y_{s}\right)} d \phi \circ\left(F_{\tilde{w}} \circ \phi^{-1}\right)\right)\left(\phi\left(y_{s}\right)\right)\right|_{w \otimes \tilde{w}=\mathbb{X}_{s, t}} \\
=\phi\left(y_{s}\right)+d \phi \circ F_{x_{s, t}}\left(y_{s}\right)+\left.F_{w}\left(y_{s}\right)\left[d \phi \circ F_{\tilde{w}}\right]\right|_{w \otimes \tilde{w}=\mathbb{X}_{s, t}} .
\end{aligned}
$$

We note that $F$ is linear with its range in the algebra of differential operators, we can extend it uniquely to $\mathcal{F}$ which acts on the tensor algebra $T\left(\mathbb{R}^{n}\right)$. In that case, we may write (5.11) more concisely as

$$
\phi\left(y_{t}\right) \underset{3}{\approx} \phi\left(y_{s}\right)+\left(\mathcal{F}_{\mathbf{X}_{s, t}} \phi\right)\left(y_{s}\right) .
$$

This approximation will be satisfied for our solution to a rough differential equation on a manifold. However, we will opt to define our solution in a coordinate-free but equivalent way: 
Definition $5.2 \mathbf{y}=\left(y, y^{\dagger}\right)$ on $I_{0}=[0, T]$ or $[0, T)$ solves $(5.8)$ if $y_{s}^{\dagger}=F_{(\cdot)}\left(y_{s}\right)$ and for every $f \in C^{\infty}(M)$ and $[a, b] \subseteq I_{0}$, the approximation

$$
f\left(y_{t}\right)-f\left(y_{s}\right) \underset{3}{\approx}\left(\mathcal{F}_{\mathbf{X}_{s, t}} f\right)\left(y_{s}\right)
$$

holds for $a \leq s \leq t \leq b$.

If in addition $y_{0}=\bar{y}_{0}$, we say $\mathbf{y}$ solves (5.8) with initial condition $y_{0}=\bar{y}_{0}$.

While this is an intuitive definition, there are many workable characterizations of solving a rough differential equation. Before presenting a few more, we note that if $\alpha \in \Omega^{1}(M, V)$ and $F: M \rightarrow L(W, T M)$ is smooth, then the composition $\alpha \circ F_{(\cdot)}$ is a smooth map from $M$ to $V$. Given $\mathbf{y} \in C R P_{\mathbf{X}}(M)$, we can then define the push-forward $\left[\alpha \circ F_{(\cdot)}\right]_{*} \mathbf{y} \in C R P_{\mathbf{X}}(L(W, V))$. Recall from Theorem 2.7 that we can define the integral increment

$$
\int_{s}^{t}\left\langle\left(\left[\alpha \circ F_{(\cdot)}\right]_{*} \mathbf{y}\right)_{\tau}, d \mathbf{X}_{\tau}\right\rangle \text {. }
$$

With this idea in mind, we now give other characterizations of solving Eq. (5.8).

Theorem 5.3 Let $y$ be a path in $M$ on $I_{0}$ with $y_{s}^{\dagger}=F$. $\left(y_{s}\right)$. Let $\mathbf{y}=\left(y, y^{\dagger}\right) \in C R P_{\mathbf{X}}(M)$. The following are equivalent.

1. For every chart $\phi$ with $a, b \in I_{0}$ such that $y([a, b]) \subseteq D(\phi)$ the approximation

$$
\phi\left(y_{t}\right) \underset{3}{\approx} \phi\left(y_{s}\right)+d \phi \circ F_{x_{s, t}}\left(y_{s}\right)+\left.F_{w}\left(y_{s}\right)\left[d \phi \circ F_{\tilde{w}}\right]\right|_{w \otimes \tilde{w}=\mathbb{X}_{s, t}}
$$

holds $a \leq s \leq t \leq b$; that is

$$
\phi\left(y_{t}\right)-\phi\left(y_{s}\right)=\int_{s}^{t}\left\langle\left(\left[d \phi \circ F_{(\cdot)}\right]_{*} \mathbf{y}\right)_{\tau}, d \mathbf{X}_{\tau}\right\rangle
$$

for $a \leq s \leq t \leq b$.

2. If $V$ is a Banach space, $\alpha \in \Omega^{1}(M, V)$, and $[a, b]$ is such that $[a, b] \subseteq I_{0}$ then

$$
\int_{s}^{t} \alpha(d \mathbf{y}) \underset{3}{\approx} \alpha\left(F_{x_{s, t}}\left(y_{s}\right)\right)+\left.F_{w}\left(y_{s}\right)\left[\alpha \circ F_{\tilde{w}}\right]\right|_{w \otimes \tilde{w}=\mathbb{X}_{s, t}}
$$

for $a \leq s \leq t \leq b$; that is

$$
\int_{s}^{t} \alpha(d \mathbf{y})=\int_{s}^{t}\left\langle\left(\left[\alpha \circ F_{(\cdot)}\right]_{*} \mathbf{y}\right)_{\tau}, d \mathbf{X}_{\tau}\right\rangle
$$

for $a \leq s \leq t \leq b$.

3. $\mathbf{y}$ solves (5.8); that is

$$
f\left(y_{t}\right)-f\left(y_{s}\right)=\int_{s}^{t}\left\langle\left(\left[d f \circ F_{(\cdot)}\right]_{*} \mathbf{y}\right)_{\tau}, d \mathbf{X}_{\tau}\right\rangle
$$

for every $f \in C^{\infty}(M)$ 
Proof. We will only prove the approximations in each case, that is the first statement of each item. The second statements are immediate from the definitions.

$(1 \Longrightarrow 2)$ We assume that $\mathbf{y}$ satisfies the approximation in Eq. 5.13 for any chart. Let $[a, b] \subseteq I_{0}$ be given. For every $m \in y([a, b])$, we have there exists a chart $\phi^{m}$ with open domain $\mathcal{V}_{m}:=D\left(\phi^{m}\right)$ containing $m$ whose range $R\left(\phi^{m}\right)$ is convex. We may now use our patching strategy outlined in Remark 2.52 with the cover $\left\{V_{m}\right\}_{m \in y([a, b])}$ applied to the function

$$
(s, t) \longrightarrow \int_{s}^{t} \alpha(d \mathbf{y})-\alpha\left(F_{x_{s, t}}\left(y_{s}\right)\right)-\left.F_{w}\left(y_{s}\right)\left[\alpha \circ F_{\tilde{w}}\right]\right|_{w \otimes \tilde{w}=\mathbb{X}_{s, t}}
$$

to reduce to the case where $y([a, b])$ is contained in the domain of a single chart.

With this reduction, we can further reduce to the flat case by defining $\mathbf{z}_{t}:=\left(\phi\left(y_{t}\right), F .\left(y_{s}\right)\right)$ and $F^{\phi}:=d \phi\left(F \circ \phi^{-1}\right)$ and showing

$$
\begin{aligned}
& \int_{s}^{t} \alpha(d \mathbf{y})-\alpha\left(F_{x_{s, t}}\left(y_{s}\right)\right)-\left.F_{w}\left(y_{s}\right)\left[\alpha \circ F_{\tilde{w}}\right]\right|_{w \otimes \tilde{w}=\mathbb{X}_{s, t}} \\
& \quad=\int_{s}^{t}\left(\left(\phi^{-1}\right)^{*} \alpha\right)(d \mathbf{z})-\left(\left(\phi^{-1}\right)^{*} \alpha\right)_{z_{s}}\left(F_{x_{s, t}}^{\phi}\left(z_{s}\right)\right)-\left.\left(\partial_{F_{w}^{\phi}\left(z_{s}\right)}\left[\left(\phi^{-1}\right)^{*} \alpha \circ F_{\tilde{w}}^{\phi}\right]\right)\left(z_{s}\right)\right|_{w \otimes \tilde{w}=\mathbb{X}_{s, t} .}
\end{aligned}
$$

The above equality is true due to the following three identities:

$$
\begin{aligned}
\int_{s}^{t} \alpha(d \mathbf{y}) & =\int_{s}^{t}\left(\left(\phi^{-1}\right)^{*} \alpha\right)(d \mathbf{z}), \\
\alpha\left(F_{x_{s, t}}\left(y_{s}\right)\right) & =\left(\left(\phi^{-1}\right)^{*} \alpha\right)_{z_{s}}\left(F_{x_{s, t}}^{\phi}\left(z_{s}\right)\right), \text { and } \\
F_{w}\left(y_{s}\right)\left[\alpha \circ F_{\tilde{w}}\right] & =\left(\partial_{F_{w}^{\phi}\left(z_{s}\right)}\left[\left(\phi^{-1}\right)^{*} \alpha \circ F_{\tilde{w}}^{\phi}\right]\right)\left(z_{s}\right) .
\end{aligned}
$$

Equation (5.14 is true by Theorem 4.15. The differential geometric identities in Eqs. 5.15 and (5.16) are simply a matter of unwinding the definitions.

$(2 \Longrightarrow 3)$ By letting $\alpha=d f$ and using Theorem 4.13 , we have

$$
\begin{aligned}
& f\left(y_{t}\right)-f\left(y_{s}\right)=\int_{s}^{t} d f(d \mathbf{y}) \\
& \quad \approx d f\left(F_{x_{s, t}}\left(y_{s}\right)\right)+\left.F_{w}\left(y_{s}\right)\left[d f \circ F_{\tilde{w}}\right]\right|_{w \otimes \tilde{w}=\mathbb{X}_{s, t}} \\
& \quad=\left(\mathcal{F}_{\mathbf{X}_{s, t}} f\right)\left(y_{s}\right)
\end{aligned}
$$

$(3 \Longrightarrow 1)$ We leave it to the reader to work through the details of this step which follow exactly as in the proof of Theorem 2.57 by letting $f^{i}$ be the coordinates of $\phi$.

By Theorem 6.9 in the Appendix, we see that a solution to a rough differential equation in flat space does actually satisfy Eq. (5.8). Moreover, we immediately get local existence of solutions:

Theorem 5.4 Let $F: W \rightarrow \Gamma(T M)$ be linear and let $\bar{y}_{0}$ be a point in $M$. There exists a local in time solution to the differential Eq. (5.8) with initial condition $y_{0}=\bar{y}_{0}$.

Proof. Let $\phi$ be any chart such that $\bar{y}_{0} \in D(\phi)$. Then there exists a solution on some time interval $[0, \tau]$ in $R(\phi)$ to the differential equation

$$
d \mathbf{z}_{t}=F_{d \mathbf{x}_{t}}^{\phi}\left(z_{t}\right)
$$


with initial condition $z_{0}=\phi\left(\bar{y}_{0}\right)$. If $\tilde{\phi}$ is any other chart such that $[a, b] \subseteq[0, \tau]$ and $y([a, b]) \subseteq$ $D(\tilde{\phi})$, then the transition map $\tilde{\phi} \circ \phi^{-1}$ has a domain containing $z([a, b])$. It is easy to check that

$$
F^{\tilde{\phi}}=\left(F^{\phi}\right)^{\tilde{\phi} \circ \phi^{-1}}
$$

and by Corollary 6.12 , after unraveling the notation, we have

$$
\tilde{\phi}\left(y_{t}\right) \underset{3}{\approx} \tilde{\phi}\left(y_{s}\right)+d \tilde{\phi} \circ F_{x_{s, t}}\left(y_{s}\right)+\left.F_{w}\left(y_{s}\right)\left[d \tilde{\phi} \circ F_{\tilde{w}}\right]\right|_{w \otimes \tilde{w}=\mathbb{X}_{s, t}}
$$

Thus satisfying the rough differential equation approximation in one chart is sufficient prove that it hold in all charts.

Solutions to rough differential equations will be unique on the intersection of their time domain up to some possible explosion time. This is stated more precisely in the following theorem.

Theorem 5.5 Let $T>0$. There is unique solution $\mathbf{y}_{t} \in C R P_{\mathbf{X}}(M)$ to $d \mathbf{y}_{t}=F_{d \mathbf{X}_{t}}\left(y_{t}\right)$ with initial condition $y_{0}=\bar{y}_{0}$ existing either on all of $[0, T]$ or on $[0, \tau)$ for some $\tau<T$ such that the closure of $\left\{y_{t}: 0 \leq t<\tau\right\}$ is not compact.

Proof. This proof follows the strategy of the proof of Theorem 4.2 in [3]. First we will show that we can always concatenate a solution $\mathbf{y}$ provided it has not exploded yet:

Suppose there exists a $\mathbf{y}$ solving $d \mathbf{y}_{t}=F_{d \mathbf{x}_{t}}\left(y_{t}\right)$ with initial condition $y_{0}=\bar{y}_{0}$ on $[0, \tau)$. If there exists a compact $K \subseteq M$ such that $\left\{y_{t}: 0 \leq t<\tau\right\} \subseteq K$, then there is a sequence of increasing times $t_{n} \in[0, \tau)$ such that $t_{n} \rightarrow \tau$ and $y_{\infty}:=\lim _{n \rightarrow \infty} y\left(t_{n}\right)$ exists and is in $K$. We can now choose a chart $\phi$ such that the closure of $D(\phi)$ is compact and such that $y_{\infty} \in D(\phi)$. Let $\mathbf{z}_{t}$ and $a$ be such that $\mathbf{z}_{t}:=\phi_{*} \mathbf{y}$ on some time interval $[a, \tau)$ such that $y([a, \tau)) \subseteq D(\phi)$. By appealing to Lemma 6.8 in the Appendix, there exists an $\epsilon>0$ and a $U \subseteq D(\phi)$ containing $y_{\infty}$ such that for any $s \in[\tau-\epsilon, \tau]$ and $\bar{z} \in U$, there exists $\tilde{\mathbf{z}} \in C R P_{\mathbf{X}}\left(\mathbb{R}^{d}\right)$ defined on $[s, \tau+\epsilon]$ which solves

$$
d \tilde{\mathbf{z}}_{t}=F_{x_{s, t}}^{\phi}\left(\tilde{z}_{t}\right) \quad \text { with } \quad \tilde{z}_{s}=\bar{z}
$$

Letting $n$ be sufficiently large, we have that $t_{n} \in[\tau-\epsilon, \tau]$ and we let $\tilde{\mathbf{z}}$ be the solution to $d \tilde{\mathbf{z}}_{t}=$ $F_{x_{s, t}}^{\phi}\left(\tilde{z}_{t}\right)$ with initial condition $\tilde{z}_{s}=z\left(t_{n}\right)$. Then we can concatenate $\mathbf{z}$ and $\tilde{\mathbf{z}}$ in the sense of Lemma 2.6. By pulling these back to the manifold by $\phi^{-1}$, we now have a solution $\tilde{\mathbf{y}}$ on $M$ which is defined on $[0, \tau+\epsilon]$.

With the preceding fact shown, we may now prove the theorem. We define

$$
\tau:=\sup \left\{T_{0} \in(0, T): \exists \mathbf{y} \text { solving } d \mathbf{y}_{t}=F_{d \mathbf{x}_{t}}\left(y_{t}\right) \text { with } y_{0}=\bar{y}_{0}\right\}
$$

We can then for any $t<\tau$ define $\mathbf{y}_{t}:=\hat{\mathbf{y}}_{t}$ where $\hat{\mathbf{y}}_{t}$ is any solution to $d \mathbf{y}_{t}=F_{d \mathbf{x}_{t}}\left(y_{t}\right)$ with initial condition $y_{0}=\bar{y}_{0}$. By the uniqueness of solutions to rough differential equations on flat space and the fact that we can cover any portion of the path with the domain of a chart, we know that $\mathbf{y}_{t}$ is well defined, and in fact satisfies $d \mathbf{y}_{t}=F_{d \mathbf{x}_{t}}\left(y_{t}\right)$ on all of $[0, \tau)$. If the closure of $\left\{y_{t}: 0 \leq t<\tau\right\}$ is compact, then from what we showed above, we can produce a solution $\tilde{\mathbf{y}}$ which is defined on $[0, \tau+\epsilon]$ for some $\epsilon>0$. In this case, $\tau$ must be $T$ and $\left.\tilde{\mathbf{y}}\right|_{[0, T]}$ is a solution defined on all of $[0, T]$. 
Definition 5.6 Let $f: M \rightarrow N$ be a smooth map between manifolds. Let $F: W \rightarrow \Gamma(T M)$ and $\tilde{F}: W \rightarrow \Gamma(T N)$ be linear. We say $F$ and $\tilde{F}$ are $f$ - related dynamical systems if

$$
f_{*} F_{w}=\tilde{F}_{w} \circ f \text { for all } w \in W .
$$

As in the flat case and shown in the Appendix in Theorem 6.11, we have a relation between dynamical systems. The proof is no different in the manifold case, and so we omit it.

Theorem 5.7 Suppose $f: M \rightarrow N$ is a smooth map between manifolds and let $F: W \rightarrow \Gamma(T M)$ and $\tilde{F}: W \rightarrow \Gamma(T N)$ be $f$-related dynamical systems. If $\mathbf{y}$ solves the initial value problem Eq. (5.8), then $\tilde{\mathbf{y}}_{t}:=\left(\tilde{y}_{t}, \tilde{y}_{s}^{\dagger}\right):=f_{*} \tilde{\mathbf{y}}_{t}$ solves

$$
d \tilde{\mathbf{y}}_{t}=\tilde{F}_{d \mathbf{X}_{t}}\left(\tilde{y}_{t}\right) \quad \text { with } \quad \tilde{y}_{0}=f\left(\bar{y}_{0}\right) .
$$

\subsubsection{RDEs from the Gauge Perspective}

Following the theme of Theorem 2.44, we also have a way to view a solution to a differential equation using the gauge perspective. Let $\psi$ be a logarithm on $M$ with diagonal domain $\mathcal{D}$.

Theorem 5.8 Let $y$ be a path in $M$ on $I_{0}$ with $y_{s}^{\dagger}=F$. $\left(y_{s}\right)$. Let $\mathbf{y}=\left(y, y^{\dagger}\right)$. Then $\mathbf{y}$ solves Equation (5.8) if and only if for every a,b such that $[a, b] \subseteq I_{0}$, there exists $a \delta>0$ such that

$$
\psi\left(y_{s}, y_{t}\right) \underset{3}{\approx} F_{x_{s, t}}\left(y_{s}\right)+\left.F_{w}\left(y_{s}\right)\left[\left(\psi_{y_{s}}\right)_{*} F_{\tilde{w}}\right]\right|_{w \otimes \tilde{w}=\mathbb{X}_{s, t}}
$$

provided $a \leq s \leq t \leq b$ and $t-s<\delta$.

Proof. This proof will be similar to the proof of Theorem 2.44

First we show the condition of Theorem 5.8 implies that $\mathbf{y}$ solves Equation 55.8 . Let $\phi$ be a chart and let $[a, b]$ be such that $y([a, b]) \subseteq D(\phi)$. By defining

$$
\begin{aligned}
z_{s} & :=\phi\left(y_{s}\right) \\
\psi^{\phi}(x, y) & :=\phi_{*} \psi\left(\phi^{-1}(x), \phi^{-1}(y)\right) \\
F_{w}^{\phi}(x) & :=d \phi\left(F_{w}\left(\phi^{-1}(x)\right)\right)
\end{aligned}
$$

and denoting $\psi^{\phi}(x, y)=\left(x, \bar{\psi}^{\phi}(x, y)\right)$, Eq. 5.17$)$, once pushed forward by $\phi$, can be written as

$$
\bar{\psi}^{\phi}\left(z_{s}, z_{t}\right) \underset{3}{\approx} F_{x_{s, t}}^{\phi}\left(z_{s}\right)+\left.\left(\partial_{F_{w}^{\phi}\left(z_{s}\right)}\left[\bar{\psi}_{z_{s}}^{\phi \prime}(\cdot) F_{\tilde{w}}^{\phi}(\cdot)\right]\right)\left(z_{s}\right)\right|_{w \otimes \tilde{w}=\mathbb{X}_{s, t}}
$$

provided $a \leq s \leq t \leq b$ and $t-s<\delta$. We then must prove that $z$ solves Eq. 5.10 for all $a \leq s \leq t \leq b$. However, by appealing to Lemma 2.48 and Lemma 6.13 of the Appendix, we only need to prove Eq. (5.10) holds for every $u$ in $[a, b]$ for $s \leq t$ in $\left(u-\delta_{u}, u+\delta_{u}\right) \cap[a, b]$ for some $\delta_{u}$. We do this now:

For any $u \in[a, b]$, let $\mathcal{W}_{u}$ be an open convex set of $z_{u}$ such that $\mathcal{W}_{u} \times \mathcal{W}_{u} \subseteq D\left(\psi^{\phi}\right)$. We then choose $\delta_{u}>0$ such that $z\left(\left[u-\delta_{u}, u+\delta_{u}\right] \cap[a, b]\right) \subseteq \mathcal{W}_{u}$ and $2 \delta_{u} \leq \delta$. We are now in the setting of Proposition 5.1 and have therefore shown $\mathbf{y}$ solves Eq. (5.8).

For the reverse implication, let $[a, b] \subseteq I_{0}$ be given. Choose $\delta>0$ such that $|t-s| \leq \delta$ for $a \leq s \leq t \leq b$ implies that $\left|\psi\left(y_{s}, y_{t}\right)\right|_{g}$ is bounded. Around every point $m$ of $y([a, b])$, there exists 
an open $\mathcal{O}_{m}$ containing $m$ such that $\mathcal{O}_{m} \times \mathcal{O}_{m} \subseteq \mathcal{D}$. Additionally for each $m$ there exists a chart $\phi^{m}$ such that $m \in D\left(\phi^{m}\right), D\left(\phi^{m}\right) \subseteq \mathcal{O}_{m}$, and $\mathcal{W}_{m}:=R\left(\phi^{m}\right)$ is convex. We may now use Remark 2.52 with the cover $\left\{\mathcal{V}_{m}\right\}_{m \in y([a, b])}$ and $D=\{(s, t): a \leq s \leq t \leq b$ and $|t-s| \leq \delta\}$ with the function

$$
(s, t) \longrightarrow \psi\left(y_{s}, y_{t}\right)-F_{x_{s, t}}\left(y_{s}\right)-\left.F_{w}\left(y_{s}\right)\left[\left(\psi_{y_{s}}\right)_{*} \circ F_{\tilde{w}}\right]\right|_{w \otimes \tilde{w}=\mathbb{X}_{s, t}} .
$$

Doing this, we have reduced to considering the case of our path being contained in the domain of a single chart $\phi$ such that $D(\phi) \times D(\phi) \subseteq \mathcal{D}$ and $R(\phi)$ is convex. By using the same definitions above for $z_{s}, F^{\phi}$, and $\psi^{\phi}$, we reduce proving

$$
\psi\left(y_{s}, y_{t}\right) \underset{3}{\approx} F_{x_{s, t}}\left(y_{s}\right)+\left.F_{w}\left(y_{s}\right)\left[\left(\psi_{y_{s}}\right)_{*} \circ F_{\tilde{w}}\right]\right|_{w \otimes \tilde{w}=\mathbb{X}_{s, t}}
$$

to the flat case

$$
\bar{\psi}^{\phi}\left(z_{s}, z_{t}\right) \underset{3}{\approx} F_{x_{s, t}}^{\phi}\left(z_{s}\right)+\left.\left(\partial_{F_{w}^{\phi}\left(z_{s}\right)}\left[\bar{\psi}_{z_{s}}^{\phi \prime}(\cdot) F_{\tilde{w}}^{\phi}(\cdot)\right]\right)\left(z_{s}\right)\right|_{w \otimes \tilde{w}=\mathbb{X}_{s, t}} \cdot
$$

This is now in the setting of Proposition 5.1 and hence we are finished.

Akin to the integral formulas, there is also a characterization of solving a differential equation which involves a gauge $(\psi, U)$.

Theorem $5.9 \mathbf{y}=\left(y, y^{\dagger}\right)$ on $I_{0}$ solves $\overline{5.8)}$ if and only if $y_{s}^{\dagger}=F_{(\cdot)}\left(y_{s}\right)$ and for all $[a, b] \subseteq I_{0}$, there exists a $\delta>0$ such that $|t-s| \leq \delta$, and $a \leq s \leq t \leq b$ implies

$$
\psi\left(y_{s}, y_{t}\right) \underset{3}{\approx} F_{x_{s, t}}\left(y_{s}\right)+\left.\left(-S_{y_{s}}^{\psi_{*}, U}\left[F_{w}\left(y_{s}\right) \otimes F_{\tilde{w}}\left(y_{s}\right)\right]+F_{w}\left(y_{s}\right)\left[U\left(y_{s}, \cdot\right) F_{\tilde{w}}\right]\right)\right|_{w \otimes \tilde{w}=\mathbb{X}_{s, t}} .
$$

Proof. This follows immediately from the product rule:

$$
\begin{aligned}
F_{w}\left(y_{s}\right)\left[\left(\psi_{y_{s}}\right)_{*} F_{\tilde{w}}\right] & =F_{w}\left(y_{s}\right)\left[\left(\psi_{y_{s}}\right)_{*(\cdot)} U\left(y_{s}, \cdot\right)^{-1} U\left(y_{s}, \cdot\right) F_{\tilde{w}}\right] \\
& =-S_{y_{s}}^{\psi_{*}, U}\left[F_{w}\left(y_{s}\right) \otimes F_{\tilde{w}}\left(y_{s}\right)\right]+F_{w}\left(y_{s}\right)\left[U\left(y_{s}, \cdot\right) F_{\tilde{w}}\right]
\end{aligned}
$$

Example 5.10 If $\nabla$ is a covariant derivative, then $\mathbf{y}$ on $I_{0}$ solves (5.8) if and only if $y_{s}^{\dagger}=F\left(y_{s}\right)$ and

$$
\exp _{y_{s}}^{-1}\left(y_{t}\right) \underset{3}{\widetilde{3}} F_{x_{s, t}}\left(y_{s}\right)+\left(\nabla_{F_{w}\left(y_{s}\right)} F_{\tilde{w}}\right)-\left.\frac{1}{2} T^{\nabla}\left[F_{w}\left(y_{s}\right) \otimes F_{\tilde{w}}\left(y_{s}\right)\right]\right|_{w \otimes \tilde{w}=\mathbb{X}_{s, t}}
$$

for $s$ and $t$ close.

\section{Appendix}

\subsection{Taylor Expansion on a Riemannian manifold}

Let $(M, g)$ be a Riemannian manifold, $\nabla$ be the Levi-Civita covariant derivative, $\exp (t v)$ be the geodesic flow, and $/ / t(\sigma)$ denote parallel translation relative to $\nabla$. Recall that Taylor's formula with integral remainder states for any smooth function $g$ on $[0,1]$, that

$$
G(1)=\sum_{k=0}^{n} \frac{1}{n !} G^{(k)}(0)+\frac{1}{n !} \int_{0}^{1} G^{(n+1)}(t)(1-t)^{n} d t .
$$


We now apply this result to $G(t):=f\left(\exp _{m}(t v)\right)$ where $f \in C^{\infty}(M), v \in T_{m} M$ and $m \in M$. To this end let $\sigma(t):=\exp (t v)$ so that $\nabla \dot{\sigma}(t) / d t=0$. It then follows that

$$
\begin{aligned}
\dot{G}(t) & =d f(\dot{\sigma}(t))=d f_{\sigma(t)}(\dot{\sigma}(t)), \\
\ddot{G}(t) & =\frac{d}{d t} d f_{\sigma(t)}(\dot{\sigma}(t))=\left(\nabla_{\dot{\sigma}(t)} d f\right)(\dot{\sigma}(t))+d f_{\sigma(t)}\left(\frac{\nabla}{d t} \dot{\sigma}(t)\right) \\
& =\left(\nabla_{\dot{\sigma}(t)} d f\right)(\dot{\sigma}(t))=(\nabla d f)(\dot{\sigma}(t) \otimes \dot{\sigma}(t)) \\
& \vdots \\
G^{(k)}(t) & =\left(\nabla^{k-1} d f\right)\left(\dot{\sigma}(t)^{\otimes k}\right)=\left(\nabla^{k-1} d f\right)(\overbrace{\dot{\sigma}(t) \otimes \cdots \otimes \dot{\sigma}(t)}^{k \text { times }}) .
\end{aligned}
$$

Therefore we may conclude that

$$
\begin{aligned}
f\left(\exp _{m}(v)\right) & =G(1)=\sum_{k=0}^{n} \frac{1}{n !} G^{(k)}(0) \\
& =f(x)+\sum_{k=1}^{n} \frac{1}{k !}\left(\nabla^{k-1} d f\right)\left(v^{\otimes k}\right)+\frac{1}{n !} \int_{0}^{1}\left(\nabla^{n} d f\right)\left(\dot{\sigma}(t)^{\otimes(n+1)}\right)(1-t)^{n} d t .
\end{aligned}
$$

Letting $n=\exp _{m}(v)$ in this formula then gives the following version of Taylor's theorem on a manifold.

Theorem 6.1 Let $f \in C^{\infty}(M)$ and $m, n \in M$ with $d_{g}(m, n)$ sufficiently small so that there exists a unique $v \in T_{m} M$ such that $|v|_{g_{m}} \leq d(m, n)$ and $n=\exp _{m}(v)$. Then we have

$$
\begin{aligned}
f(n) & =f(m)+\sum_{k=1}^{n} \frac{1}{k !}\left(\nabla^{k-1} d f\right)\left(v^{\otimes k}\right)+\frac{1}{n !} \int_{0}^{1}\left(\nabla^{n} d f\right)\left(\dot{\sigma}(t)^{\otimes(n+1)}\right)(1-t)^{n} d t \\
& =f(m)+\sum_{k=1}^{n} \frac{1}{k !}\left(\nabla^{k-1} d f\right)\left(\left[\exp _{m}^{-1}(n)\right]^{\otimes k}\right)+\frac{1}{n !} \int_{0}^{1}\left(\nabla^{n} d f\right)\left(\dot{\sigma}(t)^{\otimes(n+1)}\right)(1-t)^{n} d t
\end{aligned}
$$

where $\sigma(t)=\exp _{m}(t v)$. In particular since $|\dot{\sigma}(t)|_{g}=|v|_{g}=d_{g}(m, n)$ it follows that

$$
f(n)=f(m)+\sum_{k=1}^{n} \frac{1}{k !}\left(\nabla^{k-1} d f\right)\left(\left[\exp _{m}^{-1}(n)\right]^{\otimes k}\right)+O\left(d(m, n)^{n+1}\right) .
$$

Lemma 6.2 Let $M$ be an embedded submanifold of $W=\mathbb{R}^{k}$ and $P(m): W \rightarrow T_{m} M$ be orthogonal projection onto the tangent space. If $m, n \in M$ are close, then;

1. $P(m)\left[\exp _{m}^{-1}(n)-(n-m)\right]=O\left(|n-m|^{3}\right)$.

Moreover, $\exp _{m}^{-1}(n)-(n-m)=O\left(|n-m|^{2}\right)$

2. $U^{\nabla}(n, m)=P(m)+d P\left(\exp _{m}^{-1}(n)\right)+O\left(|n-m|^{2}\right)=P(n)+O\left(|n-m|^{2}\right)$ 
3. $P(n)-P(m)=d P\left(\exp _{m}^{-1}(n)\right)+O\left(|n-m|^{2}\right)$.

Here $U^{\nabla}(n, m)$ refers to the parallelism defined in Example 2.19 .

Proof. We will denote $v:=\exp _{m}^{-1}(n) \in T_{m} M$ and $\sigma(t)=\exp _{m}(t v)$.

For 1, we have by Taylor expansion on manifolds (Theorem 6.1) that

$$
G(n)=G(m)+d G(v)+\frac{1}{2}(\nabla d G)(v \otimes v)+\frac{1}{2} \int_{0}^{1}\left(\nabla^{2} d G\right)\left(\dot{\sigma}(t)^{\otimes 3}\right)(1-t)^{2} d t
$$

where $G \in C^{\infty}(M, W)$. Letting $G(m)=m$ as a function into $W$, we have

$$
n=m+\exp _{m}^{-1}(n)+\frac{1}{2}(\nabla P)(v \otimes v)+O\left(|v|_{g}^{3}\right) .
$$

Rearranging, we have

$$
\exp _{m}^{-1}(n)-(n-m)=-\frac{1}{2}(\nabla P)(v \otimes v)+O\left(|v|_{g}^{3}\right)
$$

so that

$$
P(m)\left[\exp _{m}^{-1}(n)-(n-m)\right]=-\frac{1}{2} P(m)(\nabla P)(v \otimes v)+O\left(|v|_{g}^{3}\right) .
$$

Note that $(\nabla P)(v \otimes v)=d P(v) v=d P(v) P(m) v$. Using the identities $d P Q-P d Q=0$ and $d P=-d Q$, where $Q=I-P$, we get that $P d P P=0$. Thus we have

$$
P(m)\left[\exp _{m}^{-1}(n)-(n-m)\right]=O\left(|v|^{3}\right) .
$$

Lastly, in a small neighborhood around $m,|v|_{g}=|m-n|+o(|m-n|)$ so that

$$
P(m)\left[\exp _{m}^{-1}(n)-(n-m)\right]=O\left(|n-m|^{3}\right)
$$

The fact that $\exp _{m}^{-1}(n)-(n-m)=O\left(|n-m|^{2}\right)$ is immediate from Eq. 6.6.

For 3, we use Taylor's theorem again this time with $G: M \longrightarrow L(W, W)$ defined by $G(n):=$ $P(n)$ to see that

$$
P(n)-P(m)=d P\left(\exp _{m}^{-1}(n)\right)+O\left(|v|^{2}\right) .
$$

As before, this is equivalent to $P(n)-P(m)=d P\left(\exp _{m}^{-1}(n)\right)+O\left(|m-n|^{2}\right)$.

Lastly for 2, Taylor applied to $G_{m}: M \longrightarrow L\left(T_{m} M, \mathbb{R}^{N}\right)$ defined by $G_{m}(n)=U^{\nabla}(n, m)$ gives

$$
U^{\nabla}(n, m)-P(m)=d G_{m}\left(\exp _{m}^{-1}(n)\right)+O\left(|m-n|^{2}\right)
$$

But

$$
\begin{aligned}
d G_{m}\left(\exp _{m}^{-1}(n)\right) & =\left.\frac{d}{d t}\right|_{0} U(\sigma(t), m) \\
& =-\left.d Q(\dot{\sigma}(t))\right|_{0} \\
& =-d Q\left(\exp _{m}^{-1}(n)\right) \\
& =d P\left(\exp _{m}^{-1}(n)\right)
\end{aligned}
$$


Thus we have

$$
U^{\nabla}(n, m)=P(m)+d P\left(\exp _{m}^{-1}(n)\right)+O\left(|m-n|^{2}\right)
$$

which is the first equality of 2 . The second equality follows trivially from this and 3.

\subsection{Equivalence of Riemannian Metrics on Compact Sets}

Proposition 6.3 Let $\pi: E \rightarrow N$ be a real rank $d<\infty$ vector bundle over a finite dimensional manifold $N$. Further suppose that $E$ is equipped with smoothly varying fiber inner product $g$ and let $S_{g}:=\{\xi \in E: g(\xi, \xi)=1\}$ be a sub-bundle of $E$. Then for any compact $K \subseteq N, \pi^{-1}(K) \cap S_{g}$ is a compact sets.

Proof. We wish to show that every sequence $\left\{\xi_{l}\right\}_{l=1}^{\infty} \subset \pi^{-1}(K) \cap S_{g}$ has a convergent subsequence. Since $\left\{\pi\left(\xi_{l}\right)\right\}_{l=1}^{\infty}$ is a sequence in $K$, by passing to a subsequence if necessary we may assume that $m:=\lim _{l \rightarrow \infty} \pi\left(\xi_{l}\right)$ exists in $K$. By passing to a further subsequence if necessary we may assume that $\left\{\xi_{l}\right\}_{l=1}^{\infty} \in \pi^{-1}\left(K_{0}\right) \cap S_{g}$ where $K_{0}$ is a compact neighborhood of $m$ which is contained in an open neighborhood $U$ over which $E$ is trivializable and hence we may now assume that $\pi^{-1}(U)=U \times \mathbb{R}^{d}$ and that $\xi_{l}=\left(n_{l}, v_{l}\right)$ where $\lim _{l \rightarrow \infty} n_{l}=m \in K_{0}$.

Let $S^{d-1}$ denote the standard Euclidean unit sphere inside of $\mathbb{R}^{d}$. The function, $F: U \times S^{d-1} \rightarrow$ $(0, \infty)$ defined by $F(n, v)=g((n, v),(n, v))$ is smooth and hence has a minimum $c>0$ and a maximum, $C<\infty$ on the compact set, $K \times S^{d-1}$. Therefore by a simple scaling argument we conclude that

$$
c|v|^{2} \leq g((n, v),(n, v)) \leq C|v|^{2} \forall n \in K \text { and } v \in \mathbb{R}^{d} .
$$

From the lower bound in Inequality (6.7) and the assumption that $1=g\left(\xi_{l}, \xi_{l}\right)$ it follows that $\left|v_{l}\right|_{\mathbb{R}^{d}} \leq 1 / \sqrt{c}$ for all $l$ and therefore has a convergent sub-sequence $\left\{v_{l_{k}}\right\}_{k=1}^{\infty}$. This completes the proof as $\left\{\xi_{l_{k}}=\left(n_{l_{k}}, v_{l_{k}}\right)\right\}_{k=1}^{\infty}$ is convergent as well.

Corollary 6.4 If $g, \tilde{g}$ are two Riemannian metrics on $T M, K \subseteq M$ is compact, then there exists $0<c_{K}, C_{K}<\infty$ such that

$$
c_{K}|v|_{\tilde{g}_{m}} \leq|v|_{g_{m}} \leq C_{K}|v|_{\tilde{g}_{m}} \forall v \in \pi^{-1}(K)
$$

In other words, all Riemannian metrics are equivalent when restricted to compact subsets, $K \subset M$.

Proof. The function, $F: T M \rightarrow[0, \infty)$, defined by $F(v):=g(v, v)$ is smooth and positive when restricted to $S_{\tilde{g}} \cap \pi^{-1}(K)$ which is compact by Proposition 6.3. Therefore there exists $0<c_{K}<C_{K}<\infty$ such that $c_{K}^{2} \leq g(v, v) \leq C_{K}^{2}$ for all $v \in S_{\tilde{g}} \cap \pi^{-1}(K)$ from which Inequality 6.8) follows by a simple scaling argument.

\subsection{Covariant Derivatives on Euclidean Space}

On $\mathbb{R}^{d}$ every covariant derivative takes the form $\nabla_{(x, v)}=\partial_{v}+A_{x}\langle v\rangle$ where $A: \mathbb{R}^{d} \rightarrow L\left(\mathbb{R}^{d}, L\left(\mathbb{R}^{d}, \mathbb{R}^{d}\right)\right)$. If $\sigma_{x}^{v}(t)=\exp _{x}(t v)$ where $\exp =\exp ^{\nabla}$, we have by definition

$$
\begin{aligned}
\partial_{\dot{\sigma}_{x}^{v}(t)} \dot{\sigma}_{x}^{v} & =-A_{\sigma_{x}^{v}(t)}\left\langle\dot{\sigma}_{x}^{v}(t)\right\rangle \dot{\sigma}_{x}^{v}(t) \\
\dot{\sigma}_{x}^{v}(0) & =v \\
\sigma_{x}^{v}(0) & =x
\end{aligned}
$$


In particular if $f_{x}=\exp _{x}(\cdot)$ plugging in at $t=0$ we get

$$
f_{x}^{\prime \prime}(0)[v \otimes v]=-A_{x}\langle v\rangle v .
$$

Now if we denote $G_{x}:=\exp _{x}^{-1}(\cdot)$ and by differentiating $f_{x} \circ G_{x}$ twice, we get that

$$
G_{x}^{\prime \prime}(x)[v \otimes v]=A_{x}\langle v\rangle v .
$$

Indeed we have

$$
\begin{aligned}
0 & =\left(f_{x} \circ G_{x}\right)^{\prime \prime}(x) \\
& =\left[f_{x}^{\prime}\left(G_{x}(x)\right) G_{x}^{\prime}(x)\right]^{\prime} \\
& =f_{x}^{\prime \prime}\left(G_{x}(x)\right)\left[G_{x}^{\prime}(x) \otimes G_{x}^{\prime}(x)\right]+f_{x}^{\prime}\left(G_{x}(x)\right) G_{x}^{\prime \prime}(x) .
\end{aligned}
$$

Since $G_{x}(x)=0, G_{x}^{\prime}(x)=I$, and $f_{x}^{\prime}(0)=I$ we have

$$
f_{x}^{\prime \prime}(0)=-G_{x}^{\prime \prime}(x)
$$

Parallel translation $U^{\nabla}\left(\sigma_{x}^{v}(t), x\right)$ solves

$$
\begin{aligned}
\frac{d}{d t} U^{\nabla}\left(\sigma_{x}^{v}(t), x\right) & =-A_{\sigma_{x}^{v}(t)}\left\langle\dot{\sigma}_{x}^{v}(t)\right\rangle U^{\nabla}\left(\sigma_{x}^{v}(t), x\right) \\
U^{\nabla}(x, x) & =I
\end{aligned}
$$

Again, using $t=0$ we have that if $\tilde{G}_{x}=U^{\nabla}(\cdot, x)$ then

$$
\tilde{G}_{x}^{\prime}(x) v=-A_{x}\langle v\rangle .
$$

To summarize, we have

$$
\left(\exp _{x}^{-1}\right)^{\prime \prime}(x)[v \otimes v]=A_{x}\langle v\rangle v
$$

and

$$
\left(U^{\nabla}(\cdot, x)\right)^{\prime}(x) v=-A_{x}\langle v\rangle .
$$

Since $\left(\exp _{x}^{-1}\right)^{\prime \prime}(x)$ is symmetric, we have that

$$
\begin{aligned}
\left(\exp _{x}^{-1}\right)^{\prime \prime}(x)[v \otimes w] & =\frac{1}{2}\left(\exp _{x}^{-1}\right)^{\prime \prime}(x)(v \otimes w+w \otimes v)+\frac{1}{2}\left(\exp _{x}^{-1}\right)^{\prime \prime}(x)(v \otimes w-w \otimes v) \\
& =\frac{1}{2}\left(\exp _{x}^{-1}\right)^{\prime \prime}(x)(v \otimes w+w \otimes v) \\
& =\frac{1}{2} A_{x}(v \otimes w+w \otimes v) \\
& =\frac{1}{2}\left(A_{x}\langle v\rangle w+A_{x}\langle w\rangle v\right)
\end{aligned}
$$

Another way of saying this is that $\left(\exp _{x}^{-1}\right)^{\prime \prime}(x)$ equals the symmetric part of $A_{x}$. By using this fact and Taylor's theorem, we get the following result. 
Lemma 6.5 If $\nabla_{(x, v)}=\partial_{v}+A_{x}\langle v\rangle$ is a covariant derivative on $\mathbb{R}^{d}$, then

$$
\begin{gathered}
\left(\exp _{x}^{\nabla}\right)^{-1}(y)-(y-x)-\frac{1}{2} A_{x}\langle y-x\rangle\langle y-x\rangle=O\left(|y-x|^{3}\right) \\
U^{\nabla}(y, x)-I+A_{x}\langle y-x\rangle=O\left(|y-x|^{2}\right)
\end{gathered}
$$

where $|x-y|$ is small enough for these terms to make sense.

Corollary 6.6 If $\nabla_{(x, v)}=\partial_{v}+A_{x}\langle v\rangle$ is a covariant derivative on $\mathbb{R}^{d}$, then

$$
U^{\nabla}(y, x)-I-A_{y}\langle x-y\rangle=O\left(|y-x|^{2}\right)
$$

where $|x-y|$ is small enough for these terms to make sense. In particular, we have

$$
\left(U^{\nabla}(x, \cdot)\right)^{\prime}(x) v=A_{x}\langle v\rangle
$$

Proof. This is immediate after expanding $A_{(\cdot)}$ about $x$ in the direction $y-x$ in Eq. 6.11 with Taylor's theorem.

\subsection{Second order gauge inequality does not imply second order chart inequality.}

Example 6.7 Let $x_{s}$ and $y_{s}$ be the $C([0,2], \mathbb{R})$ paths defined by

$$
y_{s}=x_{s}=\left\{\begin{array}{ccc}
0 & \text { if } & 0 \leq s \leq 1 \\
s^{1 / p}-1 & \text { if } & 1 \leq s \leq 2
\end{array}\right.
$$

and the control $\omega(s, t)$ be defined by

$$
\omega(s, t)=\left\{\begin{array}{ccc}
0 & \text { if } t \leq 1 \\
t-(s \vee 1) & \text { if } t \geq 1
\end{array} .\right.
$$

Then it is easy to check that

$$
\left|x_{s, t}\right| \leq \omega(s, t)^{1 / p}
$$

Let

$$
y_{s}^{\dagger}=\left\{\begin{array}{ccc}
2-2 s & \text { if } & 0 \leq s \leq \frac{1}{2} \\
1 & \text { else } & \frac{1}{2} \leq s \leq 2
\end{array} .\right.
$$

Then if $t-s \leq 1 / 2, y_{s, t}-y_{s}^{\dagger} x_{s, t}=0$ so that $\left(y, y^{\dagger}\right)$ satisfies Inequality 2.22 with $\delta=1 / 2$ and $\psi(x, y)=y-x$. On the other hand if $s=0$ and $t=1+\epsilon$, then

$$
y_{s, t}-y_{s}^{\dagger} x_{s, t}=\epsilon^{1 / p}-2 \epsilon^{1 / p}=-\epsilon^{1 / p} .
$$

Thus

$$
\frac{\left|y_{0,1+\epsilon}-y_{0}^{\dagger} x_{0,1+\epsilon}\right|}{\omega(0,1+\epsilon)^{2 / p}}=\frac{1}{\epsilon^{1 / p}}
$$

so that $\left(y, y^{\dagger}\right)$ does not satisfy Inequality 2.24 with the identity chart. 


\subsection{Rough Differential Equation Results in Euclidean Space}

The following lemma (which is Corollary 2.17 in 3 and was proved using Theorem 10.14 of [13]) proves useful in the manifold case.

Lemma 6.8 Let $U \subseteq \mathbb{R}^{d}$ be an open set and $U_{1}$ be a precompact open set whose closure is contained in $U$. There exists a $\delta>0$ such that for all $\left(\bar{z}_{0}, t_{0}\right) \in U_{1} \times[0, T]$, the rough differential equation

$$
d \mathbf{z}_{t}=F_{d \mathbf{X}_{t}}\left(z_{t}\right) \quad \text { with } \quad z_{t_{0}}=\bar{z}_{0}
$$

has a unique solution $\mathbf{z} \in C R P_{\mathbf{X}}\left(\mathbb{R}^{d}\right)$ which is defined on $\left[t_{0}, t_{0}+\delta \wedge T\right]$ with $z_{t} \in U$ for all $t \in$ $\left[t_{0}, t_{0}+\delta \wedge T\right]$.

We now state an equivalent condition for the path $\mathbf{z}$ to solve Eq. 2.11).

Theorem 6.9 Let $U \subseteq \mathbb{R}^{d}$ be open such and $\mathbf{z}=\left(z, z^{\dagger}\right) \in C R P_{\mathbf{X}}\left(\mathbb{R}^{d}\right)$ defined on $I_{0}$ such that $z\left(I_{0}\right) \subseteq U$. Then $\mathbf{z}$ solves $E q$. 2.11) if and only if $z_{s}^{\dagger}=F$. $\left(z_{s}\right)$ and for every $[a, b] \subseteq I_{0}$, Banach space $V$, and $\alpha \in \Omega^{1}(U, V)$, the approximation

$$
\int_{s}^{t} \alpha(d \mathbf{z}) \underset{3}{\approx} \alpha_{z_{s}}\left(F_{x_{s, t}}\left(z_{s}\right)\right)+\left.\left(\partial_{F_{w}\left(z_{s}\right)}\left[\alpha \circ F_{\tilde{w}}\right]\right)\left(z_{s}\right)\right|_{w \otimes \tilde{w}=\mathbb{X}_{s, t}}
$$

holds.

Proof. This is proved in 3 [Theorem 4.5 by letting $M=U$ ] but included here for completeness. To prove the "if" direction, it suffices to let $\alpha=d\left(I_{U}\right)$ and notice that

$$
\int_{s}^{t} d\left(I_{U}\right)(d \mathbf{z})=z_{t}-z_{s}
$$

by Theorem 4.13 and that $d\left(I_{U}\right)_{u}(\tilde{u})=\tilde{u}$ so that

$$
d\left(I_{U}\right)_{z_{s}}\left(F_{x_{s, t}}\left(z_{s}\right)\right)=F_{x_{s, t}}\left(z_{s}\right)
$$

and

$$
\left(\partial_{F_{w}\left(z_{s}\right)}\left[d\left(I_{U}\right) \circ F_{\tilde{w}}\right]\right)\left(z_{s}\right)=\left(\partial_{F_{w}\left(z_{s}\right)} F_{\tilde{w}}\right)\left(z_{s}\right) .
$$

To prove the "only if" direction, by definition we have

$$
z_{s, t} \underset{3}{\approx} F_{x_{s, t}}\left(z_{s}\right)+\left.\left(\partial_{F_{w}\left(z_{s}\right)} F_{\tilde{w}}\right)\left(z_{s}\right)\right|_{w \otimes \tilde{w}=\mathbb{X}_{s, t}}
$$

and

$$
\int_{s}^{t} \alpha(d \mathbf{z}) \underset{3}{\approx} \alpha_{z_{s}}\left(z_{s, t}\right)+\alpha_{z_{s}}^{\prime}\left(F .\left(z_{s}\right) \otimes F .\left(z_{s}\right) \mathbb{X}_{s, t}\right) .
$$

Combining these approximations, we have

$$
\begin{aligned}
\int_{s}^{t} \alpha(d \mathbf{z}) & \underset{3}{\approx} \alpha_{z_{s}}\left(z_{s, t}\right)+\alpha_{z_{s}}^{\prime}\left(F .\left(z_{s}\right) \otimes F .\left(z_{s}\right) \mathbb{X}_{s, t}\right) \\
& \underset{3}{\widetilde{3}} \alpha_{z_{s}}\left(F_{x_{s, t}}\left(z_{s}\right)+\left(\partial_{F_{w}\left(z_{s}\right)} F_{\tilde{w}}\right)\left(z_{s}\right)\right)+\left.\alpha_{z_{s}}^{\prime}\left(F_{w}\left(z_{s}\right) \otimes F_{\tilde{w}}\left(z_{s}\right)\right)\right|_{w \otimes \tilde{w}=\mathbb{X}_{s, t}} \\
& =\alpha_{z_{s}}\left(F_{x_{s, t}}\left(z_{s}\right)\right)+\left.\left(\partial_{F_{w}\left(z_{s}\right)}\left[\alpha \circ F_{\tilde{w}}\right]\right)\left(z_{s}\right)\right|_{w \otimes \tilde{w}=\mathbb{X}_{s, t}}
\end{aligned}
$$


where the last equality follows from the calculation

$$
\begin{aligned}
\left(\partial_{F_{w}\left(z_{s}\right)}\left[\alpha \circ F_{\tilde{w}}\right]\right)\left(z_{s}\right) & =\left(\partial_{F_{w}\left(z_{s}\right)}\left[\alpha_{z_{s}} \circ F_{\tilde{w}}(\cdot)\right]\right)\left(z_{s}\right)+\left(\partial_{F_{w}\left(z_{s}\right)} \alpha_{(\cdot)} \circ F_{\tilde{w}}\left(z_{s}\right)\right)\left(z_{s}\right) \\
& =\alpha_{z_{s}}\left(\left(\partial_{F_{w}\left(z_{s}\right)} F_{\tilde{w}}\right)\left(z_{s}\right)\right)+\alpha_{z_{s}}^{\prime}\left(F_{w}\left(z_{s}\right) \otimes F_{\tilde{w}}\left(z_{s}\right)\right)
\end{aligned}
$$

Theorem 6.11 below is useful in showing that a solution to an RDE in the flat case satisfies our manifold Definition 5.2. Let $U$ and $\tilde{U}$ be open sets for the remainder of this subsection.

Definition 6.10 Let $f: U \subseteq \mathbb{R}^{d} \rightarrow \tilde{U} \subseteq \mathbb{R}^{\tilde{d}}$ be a smooth map. Let $F: U \rightarrow L\left(W, \mathbb{R}^{d}\right)$ and $\tilde{F}: \tilde{U} \rightarrow L\left(W, \mathbb{R}^{\tilde{d}}\right)$ be smooth. We say $F$ and $\tilde{F}$ are $f$ - related dynamical systems if

$$
f^{\prime}(x) F_{w}(x)=\tilde{F}_{w} \circ f(x) \text { for all } w \in W .
$$

Theorem 6.11 Suppose $f: U \subseteq \mathbb{R}^{d} \rightarrow \tilde{U} \subseteq \mathbb{R}^{\tilde{d}}$ is a smooth map and let $F: U \rightarrow L\left(W, \mathbb{R}^{d}\right)$ and $\tilde{F}: \tilde{U} \rightarrow L\left(W, \mathbb{R}^{\tilde{d}}\right)$ be $f$-related dynamical systems. If $\mathbf{z}$ solves

$$
d \mathbf{z}_{t}=F_{d \mathbf{X}_{t}}\left(z_{t}\right)
$$

with initial condition $z_{0}=\bar{z}_{0}$, then $\tilde{\mathbf{z}}_{t}:=\left(\tilde{z}_{t}, \tilde{z}_{s}^{\dagger}\right):=f_{*} \mathbf{z}_{t}$ solves

$$
d \tilde{\mathbf{z}}_{t}=\tilde{F}_{d \mathbf{X}_{t}}\left(\tilde{z}_{t}\right)
$$

with initial condition $\tilde{z}_{0}=f\left(\bar{z}_{0}\right)$.

Proof. We have by letting $\alpha:=d f$ in Theorem 6.9

$$
\begin{aligned}
\tilde{z}_{s, t} & =f\left(z_{t}\right)-f\left(z_{s}\right) \\
& \approx f^{\prime}\left(z_{s}\right) F_{x_{s, t}}\left(z_{s}\right)+\left.\partial_{F_{w}\left(z_{s}\right)}\left[f^{\prime}(\cdot) F_{\tilde{w}}(\cdot)\right]\left(z_{s}\right)\right|_{w \otimes \tilde{w}=\mathbb{X}_{s, t}} \\
& \approx \tilde{F}_{x_{s, t}}\left(\tilde{z}_{s}\right)+\left.\left(\partial_{F_{w}\left(z_{s}\right)} \tilde{F}_{\tilde{w}} \circ f\right)\left(z_{s}\right)\right|_{w \otimes \tilde{w}=\mathbb{X}_{s, t}} \\
& \approx \tilde{F}_{x_{s, t}}\left(\tilde{z}_{s}\right)+\left.\tilde{F}_{\tilde{w}}^{\prime}\left(f\left(z_{s}\right)\right) f^{\prime}\left(z_{s}\right) F_{w}\left(z_{s}\right)\right|_{w \otimes \tilde{w}=\mathbb{X}_{s, t}} \\
& \approx \tilde{F}_{x_{s, t}}\left(\tilde{z}_{s}\right)+\left.\tilde{F}_{\tilde{w}}^{\prime}\left(f\left(z_{s}\right)\right) \tilde{F}_{w} \circ f\left(z_{s}\right)\right|_{w \otimes \tilde{w}=\mathbb{X}_{s, t}} \\
& \approx \tilde{F}_{x_{s, t}}\left(\tilde{z}_{s}\right)+\left.\left(\partial_{\tilde{F}_{w}\left(\tilde{z}_{s}\right)} \tilde{F}_{\tilde{w}}\right)\left(\tilde{z}_{s}\right)\right|_{w \otimes \tilde{w}=\mathbb{X}_{s, t} .}
\end{aligned}
$$

Additionally

$$
\tilde{z}_{t}^{\dagger}=f^{\prime}\left(z_{t}\right) z_{t}^{\dagger}=f^{\prime}\left(z_{t}\right) F_{(\cdot)}\left(z_{t}\right)=\tilde{F}_{(\cdot)}\left(\tilde{z}_{t}\right)
$$

Corollary 6.12 Let $\phi: U \subseteq \mathbb{R}^{d} \rightarrow \tilde{U} \subseteq \mathbb{R}^{d}$ be a diffeomorphism with $\phi\left(z\left(I_{0}\right)\right) \subseteq U$. Then $\mathbf{z}$ on $I_{0}$ solves

$$
d \mathbf{z}_{t}=F_{d \mathbf{x}_{t}}\left(z_{t}\right)
$$

with initial condition $z_{0}=\bar{z}_{0}$ if and only if $\tilde{\mathbf{z}}:=\phi_{*} \mathbf{z}$ on $I_{0}$ solves

$$
d \tilde{\mathbf{z}}_{t}=F_{d \mathbf{X}_{t}}^{\phi}\left(\tilde{z}_{t}\right)
$$

with initial condition $\tilde{z}_{0}=\phi\left(\bar{z}_{0}\right)$ where $F^{\phi}:=d \phi \circ\left(F \circ \phi^{-1}\right)$. 
Proof. This follows from Theorem 6.11 by seeing that $F$ is $\phi$-related to $F^{\phi}$.

This last lemma helps patch solutions in the manifold case.

Lemma 6.13 Let $z \in C([0, T], V)$ and let $0=t_{0}<t_{1}<\ldots<t_{l}=T$ be a partition of $[0, T]$. If

$$
z_{s, t} \underset{3}{\approx} F_{x_{s, t}}\left(z_{s}\right)+\left.\left(\partial_{F_{w}\left(z_{s}\right)} F_{\tilde{w}}\right)\left(z_{s}\right)\right|_{w \otimes \tilde{w}=\mathbb{X}_{s, t}}
$$

holds for all $t_{i} \leq s \leq t \leq t_{i+1}$ and $0 \leq i<l$ then Eq. 6.12) holds for $0 \leq s \leq t \leq T$.

In particular, if $\mathbf{z}_{t}$ solves $d \mathbf{z}_{t}=F_{d \mathbf{X}_{t}}\left(z_{t}\right)$ with $z_{0}=\bar{z}_{0}$ on $[0, \tau]$ and $\tilde{\mathbf{z}}_{t}$ solves $d \tilde{\mathbf{z}}_{t}=F_{d \mathbf{X}_{t}}\left(\tilde{z}_{t}\right)$ with $\tilde{z}_{\tau}=z_{\tau}$ on $[\tau, T]$, then the concatenation of $\mathbf{z}_{t}$ and $\tilde{\mathbf{z}}_{t}$ in the sense of Lemma 2.6 solves $d \mathbf{z}_{t}=F_{d \mathbf{x}_{t}}\left(z_{t}\right)$ with $z_{0}=\bar{z}_{0}$ on $[0, T]$.

Proof. This proof is identical from [3] [Lemma A.2], adapted here with different notation. We will only prove it in the case of two subintervals. First note that

$$
F_{w}(y)=F_{w}(x)+F_{w}^{\prime}(x)(y-x)+O\left(|w||y-x|^{2}\right)
$$

and

$$
\left(\partial_{F_{w}(y)} F_{\tilde{w}}\right)(y)=\left(\partial_{F_{w}(x)} F_{\tilde{w}}\right)(x)+O(|w||\tilde{w}||y-x|)
$$

by Taylor's theorem and the fact that $w \rightarrow F_{w}$ is linear. Using these facts, we have

$$
\begin{aligned}
z_{s, t} & =z_{s, \tau}+z_{\tau, t} \\
& \approx F_{x_{s, \tau}}\left(z_{s}\right)+\left.\left(\partial_{F_{w}\left(z_{s}\right)} F_{\tilde{w}}\right)\left(z_{s}\right)\right|_{w \otimes \tilde{w}=\mathbb{X}_{s, \tau}}+F_{x_{\tau, t}}\left(z_{\tau}\right)+\left.\left(\partial_{F_{w}\left(z_{\tau}\right)} F_{\tilde{w}}\right)\left(z_{\tau}\right)\right|_{w \otimes \tilde{w}=\mathbb{X}_{\tau, t}} \\
& \approx F_{x_{s, t}}\left(z_{s}\right)+F_{x_{\tau, t}}^{\prime}\left(z_{s}\right)\left(z_{s, \tau}\right)+\left.\left(\partial_{F_{w}\left(z_{s}\right)} F_{\tilde{w}}\right)\left(z_{s}\right)\right|_{w \otimes \tilde{w}=\mathbb{X}_{s, \tau}}+\left.\left(\partial_{F_{w}\left(z_{s}\right)} F_{\tilde{w}}\right)\left(z_{s}\right)\right|_{w \otimes \tilde{w}=\mathbb{X}_{\tau, t}} \\
& \approx F_{x_{s, t}}\left(z_{s}\right)+F_{x_{\tau, t}}^{\prime}\left(z_{s}\right)\left(F_{x_{s, \tau}}\left(z_{s}\right)\right)+\left.\left(\partial_{F_{w}\left(z_{s}\right)} F_{\tilde{w}}\right)\left(z_{s}\right)\right|_{w \otimes \tilde{w}=\mathbb{X}_{s, \tau}+\mathbb{X}_{\tau, t}} \\
& =F_{x_{s, t}}\left(z_{s}\right)+\left.\left(\partial_{F_{w}\left(z_{s}\right)} F_{\tilde{w}}\right)\left(z_{s}\right)\right|_{w \otimes \tilde{w}=\mathbb{X}_{s, \tau}+\mathbb{X}_{\tau, t}+x_{s, \tau} \otimes x_{\tau, t}} \\
& =F_{x_{s, t}}\left(z_{s}\right)+\left.\left(\partial_{F_{w}\left(z_{s}\right)} F_{\tilde{w}}\right)\left(z_{s}\right)\right|_{w \otimes \tilde{w}=\mathbb{X}_{s, t}} .
\end{aligned}
$$

\section{References}

[1] J. Blanchet, X. Chen, and J. Dong, $\epsilon$-Strong Simulation for Multidimensional Stochastic Differential Equations via Rough Path Analysis, ArXiv e-prints (2014).

[2] H. Boedihardjo, X. Geng, and Z. Qian, Quasi-sure existence of gaussian rough paths and large deviation principles for capacities, ArXiv e-prints (2013).

[3] T. Cass, B. K. Driver, and C. Litterer, Constrained Rough Paths, ArXiv e-prints (2014).

[4] Thomas Cass and Peter Friz, Densities for rough differential equations under Hörmander's condition, Ann. of Math. (2) 171 (2010), no. 3, 2115-2141. MR 2680405 (2011f:60109)

[5] Thomas Cass, Peter Friz, and Nicolas Victoir, Non-degeneracy of Wiener functionals arising from rough differential equations, Trans. Amer. Math. Soc. 361 (2009), no. 6, 3359-3371. MR 2485431 (2010g:60084) 
[6] Thomas Cass, Martin Hairer, Christian Litterer, and Samy Tindel, Smoothness of the density for solutions to gaussian differential equations, Ann. Prob., forthcoming, 2013.

[7] Thomas Cass, Christian Litterer, and Terry Lyons, Rough paths on manifolds, New trends in stochastic analysis and related topics, Interdiscip. Math. Sci., vol. 12, World Sci. Publ., Hackensack, NJ, 2012, pp. 33-88. MR 2920195

[8] _ Integrability and tail estimates for gaussian rough differential equations, Ann. Probab. 41 (2013), no. 4, 3026-3050.

[9] F. Delarue and R. Diel, Rough paths and 1d sde with a time dependent distributional drift. Application to polymers, ArXiv e-prints (2014).

[10] K. D. Elworthy, Stochastic differential equations on manifolds, London Mathematical Society Lecture Note Series, vol. 70, Cambridge University Press, Cambridge-New York, 1982. MR 675100 (84d:58080)

[11] Michel Émery, Stochastic calculus in manifolds, Universitext, Springer-Verlag, Berlin, 1989, With an appendix by P.-A. Meyer. MR 1030543 (90k:58244)

[12] Peter K. Friz and Martin Hairer, A course on rough paths, Universitext, Springer, Cham, 2014, With an introduction to regularity structures. MR 3289027

[13] Peter K. Friz and Nicolas B. Victoir, Multidimensional stochastic processes as rough paths, Cambridge Studies in Advanced Mathematics, vol. 120, Cambridge University Press, Cambridge, 2010, Theory and applications. MR 2604669

[14] M. Gubinelli, Controlling rough paths, J. Funct. Anal. 216 (2004), no. 1, 86-140. MR MR2091358 (2005k:60169)

[15] M. Hairer, A theory of regularity structures, Invent. Math. 198 (2014), no. 2, 269-504. MR 3274562

[16] Elton P. Hsu, Stochastic analysis on manifolds, Graduate Studies in Mathematics, vol. 38, American Mathematical Society, Providence, RI, 2002. MR 1882015 (2003c:58026)

[17] Nobuyuki Ikeda and Shinzo Watanabe, Stochastic differential equations and diffusion processes, second ed., North-Holland Mathematical Library, vol. 24, North-Holland Publishing Co., Amsterdam; Kodansha, Ltd., Tokyo, 1989. MR 1011252 (90m:60069)

[18] Y. Inahama, Short time kernel asymptotics for rough differential equation driven by fractional Brownian motion, ArXiv e-prints (2014).

[19] D. Kelly and I. Melbourne, Smooth approximation of stochastic differential equations, ArXiv e-prints (2014).

[20] Terry Lyons, Differential equations driven by rough signals. I. An extension of an inequality of L. C. Young, Math. Res. Lett. 1 (1994), no. 4, 451-464. MR 96b:60150

[21] _ The interpretation and solution of ordinary differential equations driven by rough signals, Stochastic analysis (Ithaca, NY, 1993), Amer. Math. Soc., Providence, RI, 1995, pp. 115128. MR 96d:34076 
[22] _ Differential equations driven by rough signals, Rev. Mat. Iberoamericana 14 (1998), no. 2, 215-310. MR 2000c:60089 


\section{Contact Information}

Bruce K. Driver

Department of Mathematics, 0112

University of California, San Diego

9500 Gilman Drive

La Jolla, California 92093-0112 USA

E-mail address: bdriver@ucsd.edu

Jeremy S. Semko

Department of Mathematics, 0112

University of California, San Diego

9500 Gilman Drive

La Jolla, California 92093-0112 USA

E-mail address: jsemko@ucsd.edu 